\title{
A Review on the Applications of Non-gray Gas Radiation Models in Multi-dimensional Systems
}

\author{
Shu Zheng, ${ }^{1}$ Ran Sui, ${ }^{2}$ Yujia Sun, ${ }^{3}$ Yu Yang ${ }^{1}$ and Qiang $\mathrm{Lu}^{1, *}$
}

\begin{abstract}
Keeping gas radiation is a major way of heat transfer in flames. It affects temperature distributions and hence causes energy transfer in the combustion gaseous as well as subsequent chemical reactions. Accurate and efficient modeling of radiative heat transfer in multi-dimensional combustion systems is challenging, due to the drastic and rapid change of the radiative properties of the reacting gases in the whole spectrum, and the extensive computational cost for solving the radiative transfer equation (RTE) in multi-dimensional space. Several gas radiation models and RTE solution methods have been proposed to treat non-gray radiation heat transfer in combustion systems. In this paper, we first review the development of spectral line databases, gas radiation models and RTE solution methods. Subsequently, the development of radiation model parameters for different gaseous species is discussed. Next, recent simulation investigations are reviewed for one-dimensional, twodimensional and three-dimensional systems involving these state-of-the-art radiation models. In addition, we also discuss machine learning approaches for establishing gas radiation models and solving RTEs in non-gray gas radiative problems, an alternative and flexible way to deal with the complex and dynamic systems. Hopefully, this review will provide an up-to-date knowledge about the numerical calculations of gas radiation heat transfers in combustion systems.
\end{abstract}

Keywords: Radiative heat transfer; Non-gray gas models; RTE; Machine learning.

Received: 1 November 2020; Accepted date: 06 February 2021.

Article type: Review Article.

\section{Introduction}

Radiation, conduction and convection are the three basic heat transfer mechanisms. For radiative heat transfer, the exchange of energy is proportional to the difference of the fourth power of temperature, which is drastically accentuated with rising temperatures than conduction and convection. Therefore, thermal radiation becomes more important and may be even dominant over conduction and convection at high temperatures. Examples include high temperature heat exchanger ${ }^{[1]}$ solar energy utilization, ${ }^{[2]}$ greenhouse gases emission, ${ }^{[3]}$ rocket propulsion, ${ }^{[4]}$ nuclear reaction, ${ }^{[5]}$ glass melting processing, ${ }^{[6]}$ pulsed laser ${ }^{[7]}$ and other technical areas. The scope of thermal radiation comprises surface radiation, particulate radiation, gas radiation, radiative properties, inverse radiation problem and nanoscale radiative transfer.

\footnotetext{
${ }^{I}$ National Engineering Laboratory for Biomass Power Generation

Equipment, North China Electric Power University, Beijing 102206, China.

${ }^{2}$ Department of Mechanical and Aerospace Engineering, Missouri University of Science and Technology, Rolla, MO 65409, USA.

${ }^{3}$ School of Atmospheric Physics, Nanjing University of Information

Science and Technology, Nanjing 210044, China.

*Email: qianglu@mail.ustc.edu.cn (Q. Lu)
}

Gas radiative heat transfer plays a key role in combustion systems such as industrial furnaces, boilers, gas turbine combustion chambers, as well as small-scale flames. ${ }^{[8-12]}$ Different from the radiative transfer characteristics of surfaces and particles that do not vary with wavelength radically as gases, the radiative properties of gases change rapidly in the whole spectrum. ${ }^{[13]}$ Recently, with the development in aerospace, atmospheric interceptor and infrared detection, ${ }^{[14]}$ infrared gas thermal radiation characteristics and transmission mechanism has been paid wide attention. It is challenging to calculate gas radiation heat transfer in combustion systems due to two difficulties, i.e., the establishment of efficient and accurate gas radiation models, and accurate and effective methods for solving the radiative transfer equations (RTE) of multi-dimensional spaces.

The interaction between gas and thermal radiation comprises mainly emission and absorption, and also weak scattering effect. In this paper, we only review the radiative participatory media with pure emission and absorption. The emission and absorption of thermal radiation energy of a gas is related to the release and increase of molecular and atomic energy. The total energy of a gas molecule is the sum of translational, vibrational, rotational and electronic energies. ${ }^{[15]}$ 
According to the fundamental postulates of quantum mechanics, these energies cannot be evaluated continuously, but only on a series of discrete energy levels. When a molecule transits between two energy levels, it emits or absorbs a photon whose energy is equal to the difference between the two energy levels. The absorption of photons by the gas causes a dark line in the continuous spectrum while the emission of photons causes a bright line; "spectral line" is used to describe this phenomenon. The spectral lines with the same pair of vibrational energy levels are named "spectral band" and the spectral bands with the same pair of electronic energy levels are called "spectral system". The establishment of the gas radiation model and the determination of the radiative parameters depend on the spectral lines of gas molecules.

In the past decades, with the development of high resolution spectrometers, especially fourier transform infrared spectrometer (FTIR), it has become possible to measure the detailed information of spectral lines, including line intensity, line shape and line broadening. The first spectroscopic database was established by the U.S. Air Force Cambridge Research Laboratories in $1960,{ }^{[16]}$ maintained and updated by the Harvard-Smithsonian Center for astrophysics, ${ }^{[17-19]}$ named high-resolution transmission (HITRAN) database. ${ }^{[20]}$ There are only 7 species $\mathrm{H}_{2} \mathrm{O}, \mathrm{CO}_{2}, \mathrm{O}_{3}, \mathrm{~N}_{2} \mathrm{O}, \mathrm{CO}, \mathrm{CH}_{4}$ and $\mathrm{O}_{2}$ in the first version of HITRAN, while the latest version HITRAN $2016^{[21]}$ includes detailed information of 49 species. Nonetheless, the HITRAN database contains only a small number of high temperature spectral line parameters and is hence only applicable to low temperature objects such as atmospheric radiation transfer or engineering scenarios below $1000 \mathrm{~K}$. To satisfy the requirements of calculating engineering applications at high temperatures, the HITRAN research team developed the HITEMP database on the basis of the original database. The first version HITEMP 1995[22] included 4 species $\mathrm{H}_{2} \mathrm{O}, \mathrm{CO}_{2}, \mathrm{CO}$ and $\mathrm{OH}$. Comparing to HITRAN, HITEMP provides more molecular "hot line" data, spectral lines of which are insignificant at low temperatures but cannot be ignored at high temperatures. Therefore, the HITEMP database is more suitable for the calculations of applications at high temperatures. However, the emissivity of $\mathrm{CO}_{2}$ calculated by the HITEMP database is still larger than experimental data when the temperature exceeds $2000 \mathrm{~K}$. Subsequently, Russian researchers developed the Carbon Dioxide Spectroscopic Databank (CDSD) database ${ }^{[23-25]}$ containing detailed spectral line parameters only for $\mathrm{CO}_{2}$. The latest version CDSD 4000 contains 628 million spectral lines and the calculation accuracy of radiative heat transfer when the temperature is less than $4000 \mathrm{~K}$ can be guaranteed. Other researchers also developed high temperature spectral databases for $\mathrm{H}_{2} \mathrm{O}$ : AMES database (300 million spectral lines), ${ }^{[26]}$ SCAN database (3000 million spectral lines) ${ }^{[27]}$ and BT2 database (500 million spectral lines). ${ }^{[28]}$ Based on the above databases, the latest version HITEMP $2010^{[29]}$ has been developed with the newly added CN species. HITEMP 2010 contains 110 million spectral lines of $\mathrm{CO}_{2}$ and 1110 million spectral lines of $\mathrm{H}_{2} \mathrm{O}$, and showed a good performance when the temperature is less than $3000 \mathrm{~K}^{.}{ }^{[30]}$ Table $1^{[31,32]}$ shows the number of spectral lines for $\mathrm{CO}_{2}$ and $\mathrm{H}_{2} \mathrm{O}$ over the whole spectra and the selective spectral coverages in HITRAN 2004, 2008, 2012, 2016, HITEMP 1995, 2010 and CDSD 1000 and 4000 databases. It can be seen that the number of spectral lines increased significantly along with the development of different databases.

Table 1. Number of spectral lines and the spectral coverage ranges in HITRAN, HITEMP and CDSD databases for $\mathrm{CO}_{2}$ and $\mathrm{H}_{2} \mathrm{O} \cdot{ }^{[31,32]}$

\begin{tabular}{|c|c|c|c|c|}
\hline Database & $\mathrm{H}_{2} \mathrm{O}$ & $\begin{array}{l}\text { Spectral } \\
\text { coverage }\end{array}$ & $\mathrm{CO}_{2}$ & $\begin{array}{l}\text { Spectral } \\
\text { coverage }\end{array}$ \\
\hline HITRAN & 305,752 & $\begin{array}{l}0-25,710 \\
\mathrm{~cm}^{-1}\end{array}$ & 559,874 & $\begin{array}{l}0-14,075 \\
\mathrm{~cm}^{-1}\end{array}$ \\
\hline 2016 & 198,626 & $\begin{array}{l}150-9300 \\
\mathrm{~cm}^{-1}\end{array}$ & 551,551 & $\begin{array}{l}150-9300 \\
\mathrm{~cm}^{-1}\end{array}$ \\
\hline HITRAN & 224,515 & $\begin{array}{l}0-25,710 \\
\mathrm{~cm}^{-1}\end{array}$ & 471,847 & $\begin{array}{l}0-12,784 \\
\mathrm{~cm}^{-1}\end{array}$ \\
\hline 2012 & 125,143 & $\begin{array}{l}150-9300 \\
\mathrm{~cm}^{-1}\end{array}$ & 466,843 & $\begin{array}{l}346-9300 \\
\mathrm{~cm}^{-1}\end{array}$ \\
\hline HITRAN & 69,201 & $\begin{array}{l}0-25,232 \\
\mathrm{~cm}^{-1}\end{array}$ & 314,919 & $\begin{array}{l}0-12,784 \\
\mathrm{~cm}^{-1}\end{array}$ \\
\hline 2008 & 43,433 & $\begin{array}{l}150-9300 \\
\mathrm{~cm}^{-1}\end{array}$ & 310,948 & $\begin{array}{l}352-9300 \\
\mathrm{~cm}^{-1}\end{array}$ \\
\hline HITRAN & 63,196 & $\begin{array}{l}0-25,232 \\
\mathrm{~cm}^{-1}\end{array}$ & 62,913 & $\begin{array}{l}0-12,784 \\
\mathrm{~cm}^{-1}\end{array}$ \\
\hline 2004 & 43,435 & $\begin{array}{l}150-9300 \\
\mathrm{~cm}^{-1}\end{array}$ & 61,924 & $\begin{array}{l}442-8309 \\
\mathrm{~cm}^{-1}\end{array}$ \\
\hline HITEMP & $114,241,164$ & $\begin{array}{l}0-30,000 \\
\mathrm{~cm}^{-1}\end{array}$ & $11,193,608$ & $\begin{array}{l}5-12,785 \\
\mathrm{~cm}^{-1}\end{array}$ \\
\hline 2010 & $104,508,960$ & $\begin{array}{l}150-9300 \\
\mathrm{~cm}^{-1}\end{array}$ & $11,159,843$ & $\begin{array}{l}150-9300 \\
\mathrm{~cm}^{-1}\end{array}$ \\
\hline HITEMP & $1,283,468$ & $\begin{array}{l}0-24,990 \\
\mathrm{~cm}^{-1}\end{array}$ & $1,032,269$ & $\begin{array}{l}157-9648 \\
\mathrm{~cm}^{-1}\end{array}$ \\
\hline 1995 & $1,033,344$ & $\begin{array}{l}150-9300 \\
\mathrm{~cm}^{-1}\end{array}$ & $1,032,102$ & $\begin{array}{l}157-8309 \\
\mathrm{~cm}^{-1}\end{array}$ \\
\hline $\begin{array}{l}\text { CDSD- } \\
4000\end{array}$ & - & - & $565,566,452$ & $\begin{array}{l}226-8310 \\
\mathrm{~cm}^{-1}\end{array}$ \\
\hline $\begin{array}{l}\text { CDSD- } \\
1000\end{array}$ & - & - & 360,252 & $\begin{array}{l}263-9648 \\
\mathrm{~cm}^{-1}\end{array}$ \\
\hline
\end{tabular}

For a certain wavelength in the spectrum, the exact gas absorption coefficient is the superposition of the absorption characteristics of all the spectral lines at that wavelength. It is difficult to calculate gas absorption coefficients due to the absorption effect of each spectral line in the full spectrum and the existence of millions of spectral lines. According to the wavelength interval, the gas radiative properties models can be classified into the line-by-line (LBL) model, narrow band models, wide band models and global models. ${ }^{[33]}$ At each 


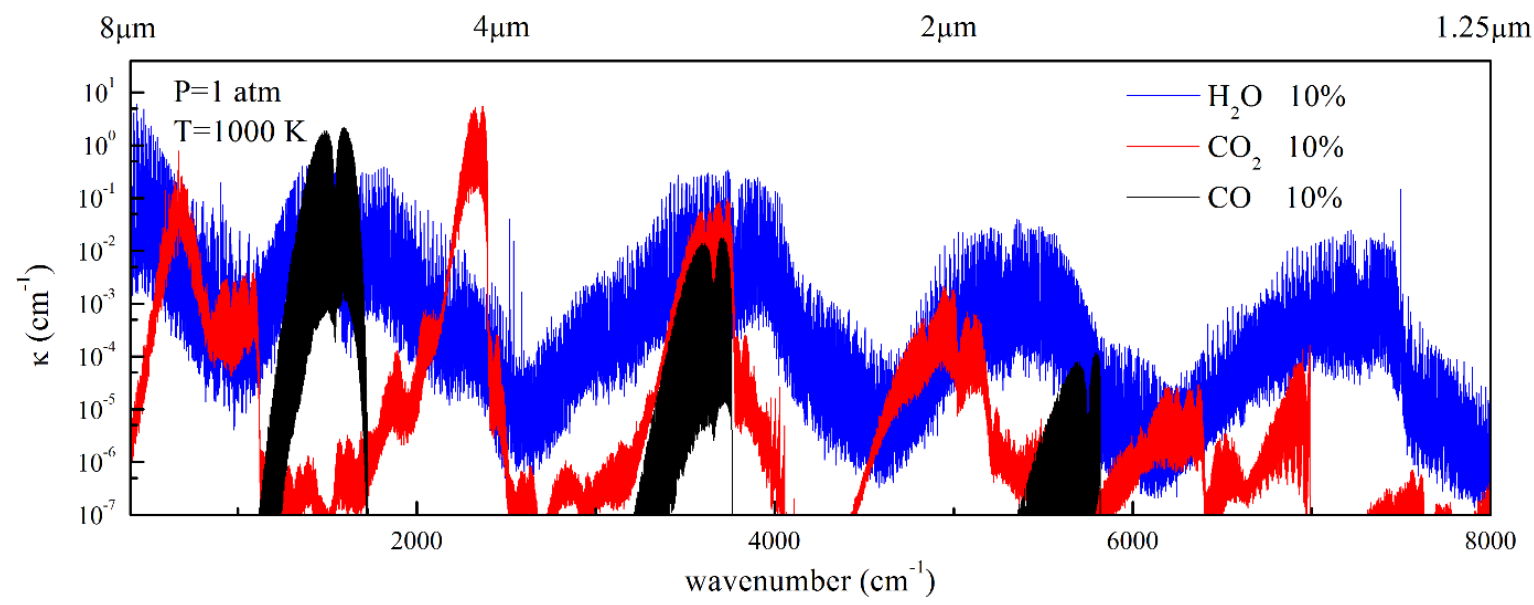

Fig. 1 Distributions of absorption coefficient calculated by LBL model along an isothermal and homogeneous path: $X_{\mathrm{H} 2 \mathrm{O}}=0.1$, $X_{\mathrm{CO} 2}=0.1, X_{\mathrm{CO}}=0.1, T=1000 \mathrm{~K}$ and $P=1 \mathrm{~atm}$.

sampling point of LBL, ${ }^{[34]}$ the absorption characteristics of all contributed spectral lines are superimposed and summed to obtain the absorption coefficient, which changes with the spectrum as shown in Fig. 1. The LBL can be used for preparing narrow band model parameters ${ }^{[35]}$ and global models validation. ${ }^{[36,37]}$ Typically, the spectral resolution in LBL simulations of gas radiative transfer is $0.01 \mathrm{~cm}^{-1}$, which requires one to solve approximately $10^{6}$ RTEs to cover the entire spectrum of interest for radiative heat transfer. ${ }^{[38]}$ Therefore, the LBL model cannot be applied to the calculations of engineering applications, and its calculation results are usually used as the benchmark to verify the accuracy of other gas radiation models.

To develop accurate and efficient non-gray gas radiation models, a number of band models were proposed some 40-50 years ago. A band model deals with the gas radiative properties in a relatively wide range of spectral intervals and the average absorption coefficient of the band is used to replace the actual value. A band model is a semi-empirical or via fitting formula, with its spectral line parameters simplified based on assumptions, not real spectral line parameters. According to the size of the spectral band interval, band models are divided into narrow band models $\left(5-50 \mathrm{~cm}^{-1}\right)$ and wide band models $\left(100-1000 \mathrm{~cm}^{-1}\right)$. Elsasser model ${ }^{[39]}$ is the simplest narrow band model, in which equally Lorenz spaced lines of equal half-width and intensity are considered. Golden $^{[40,41]}$ subsequently expanded it to utilize the Doppler and Voigt lines. Another narrow band model is the statistical narrow band model (SNB), in which the spectral lines are assumed to have random spacing and/or intensity. Based on the different intensity distributions of the spectral lines, SNB can be divided into uniform statistical model, Goody model ${ }^{[42]}$ and Malkmus model. ${ }^{[43]}$ The spectral lines in the uniform statistical model have the same intensity, while considering the change in line intensity, Goody ${ }^{[42]}$ proposed a probability distribution exponential form function to describe the line properties. Malkmus ${ }^{[43]}$ further modified the probability distribution proposed by Godson ${ }^{[44]}$ to obtain an exponentialtailed distribution function. Ludwig et al..$^{[4]}$ obtained the parameters of Goody model through experiments and Grosshandler et al. ${ }^{[46]}$ developed the RADCAL code for calculating non-gray radiative heat transfers and emissivities of gases and soots based on the Goody model and Ludwig's work. Soufiani et al..$^{[47,48]}$ proposed new narrow band parameters, which are independent of the SNB model type and applicable to both the Goody and Malkmus models.

Malkmus model is the most commonly used SNB model. Taine et al ${ }^{[3,49]}$ first generated the Malkmus SNB parameters for $\mathrm{H}_{2} \mathrm{O}$ based on HITRAN 1992 and found the maximum error was about $10 \%$ compared with LBL when the bandwidth was $25 \mathrm{~cm}^{-1}$. For an inhomogeneous gas-particle mixture, Zhang et al. ${ }^{[50]}$ studied radiative heat transfer in a finite axisymmetric system using the Malkmus model and CurtisGodson approximation. Kim et al. ${ }^{[51]}$ developed a onedimensional (1D) non-gray gas radiative transfer equation solver based on the radiative parameters of Hartmann et al. ${ }^{[35,47]}$ and Malkmus SNB model. Subsequently, the SNB model was further studied for the coupling between radiation and combustion problems. Daguse ${ }^{[52]}$ calculated the radiative properties of $\mathrm{H}_{2} \mathrm{O}$ and $\mathrm{OH}$ using Malkmus SNB model and analyzed the radiative effect on the flame structure and extinction limits of hydrogen counterflow flames. Ju et al. ${ }^{[53]}$ proposed new Planck mean absorption coefficients of $\mathrm{CO}$, $\mathrm{CO}_{2}$ and $\mathrm{H}_{2} \mathrm{O}$ using Malkmus SNB model and investigated numerically the radiation effects of Lewis number on flame bifurcation and extinction of $\mathrm{CH}_{4} / \mathrm{O}_{2} / \mathrm{N}_{2} / \mathrm{He}$ planar flames. For calculating the transmissivities of inhomogeneous gas paths, Bharadwaj and Modes ${ }^{[54]}$ developed a multi-scale Malkmus model (MSM), which performed better than the CurtisGodson approximation and correlated- $k$ model. Riviere et al. ${ }^{[5]}$ determined the Malkmus SNB parameters for $\mathrm{H}_{2} \mathrm{O}$ at high temperatures up to $2500 \mathrm{~K}$ based on HITRAN 1992 and 
experimental data. In addition, Soufiani and Taine ${ }^{[56]}$ reported the narrow band parameters of $\mathrm{H}_{2} \mathrm{O}, \mathrm{CO}_{2}$ and $\mathrm{CO}$ based on EM2C laboratory data at a spectral interval of $25 \mathrm{~cm}^{-1}$ from 150 to $9300 \mathrm{~cm}^{-1}$ at temperatures up to $2500 \mathrm{~K}$. With the development of spectroscopic databases, Riviere and Soufiani ${ }^{[57]}$ updated SNB parameters of $\mathrm{H}_{2} \mathrm{O}, \mathrm{CO}_{2}, \mathrm{CO}$ and $\mathrm{CH}_{4}$ based on CDSD-4000 and HITEMP 2010 in wide temperature and spectral ranges. To study the hydrocarbon fuels radiation reabsorption in fire applications, Consalvi and $\mathrm{Liu}^{[58]}$ established an SNB parameter database for nine fuels (methane, methanol, ethane, ethylene, propane, propylene, heptane, methyl methacrylate and toluene) based on EM2C NB and HITRAN 2012. Recently, Qi et al. ${ }^{[59]}$ generated the SNB model parameters of $\mathrm{C}_{2} \mathrm{H}_{2}$ and $\mathrm{C}_{2} \mathrm{H}_{4}$ based on HITRAN 2012 for estimating the radiative contribution of $\mathrm{C}_{2} \mathrm{H}_{2}$ and $\mathrm{C}_{2} \mathrm{H}_{4}$ in the early stage of combustion.

Generally, gas radiative properties are described by the transmissivities in the SNB models, which are not suitable for arbitrary RTE solver, and thus, some additional approximate assumptions are needed in inhomogeneous cases where errors may be caused. For this purpose, Lacis and Oinas ${ }^{[60]}$ proposed a statistical narrow band correlated- $k$ (SNBCK) model that gives a $k$ distribution of absorption coefficients within a given narrow band interval based on the SNB model. In SNBCK, the blackbody radiation intensity is independent of the wavelength The absorption coefficient with the same value may reused at different wavelengths in the narrow band, which is then reordered as a smooth and monotonically increasing function, thus ensuring each radiative intensity is calculated only once. Liu et al. ${ }^{[61]}$ studied the effects of Gauss-Legendre quadrature points on the accuracy and efficiency of SNBCK in $\mathrm{CO}_{2} / \mathrm{H}_{2} \mathrm{O} / \mathrm{N}_{2}$ mixtures under various conditions. They found the radiative source calculated by the 7 point Gauss-Legendre quadrature in SNBCK agreed well with the SNB results, while 4 point Gauss-Legendre quadrature showed the same accuracy with 7 point Gauss-Legendre quadrature but higher efficiency. The most time-consuming part in the SNBCK model is the inversion of the cumulative $k$ distribution function. Compared with the narrow band model, the large spectral band interval is used in the wide band model to reduce the computing time. There are three wide band models, i.e., the box model, ${ }^{[62]}$ the exponential wide band model (EWB) ${ }^{[63]}$ and the wide band correlated- $k$ model (WBCK). ${ }^{[64]}$ In the box model, the wide band is approximated by a rectangular box with an equivalent band width and mean absorption coefficient. EWB developed by Edwards and Menard, ${ }^{[65]}$ was by far the most successful of the wide band models. They evaluated the band absorptance using the general statistical model and carrying out the integration in an approximate fashion. To optimize the radiative property calculations of gasmixtures in the EWB, Lallemant and Weber ${ }^{[66]}$ introduced the simplifications into the calculations of the line-width-tospacing parameter with no decrement of the accuracy of the EWB, while reducing the computing time. Liu et al. ${ }^{[64]}$ developed WBCK by lumping several successive narrow bands into a wideband based on SNBCK. The maximum error of radiative source calculated by WBCK is only $3 \%$ compared with SNB. Instead of solving the RTE multiple times for each narrow band in the wideband, the radiative transfer over the wideband is calculated by concentrating several successive narrow bands into a single wideband. In general, the narrower bands are lumped, the more efficient it is; however, at the cost of accuracy as the Planck blackbody function cannot be treated satisfactorily as constant with wide bandwidth.

In the calculations of radiative heat transfer for engineering applications, usually only the total radiative heat flux was needed. A global model attempts to calculate these total heat fluxes directly using the spectral integrated radiation characteristics. The Hottel emissivity chart ${ }^{[67]}$ is a typical early global model, where the total emissivity of $\mathrm{H}_{2} \mathrm{O}$ and $\mathrm{CO}_{2}$ can be read directly from charts or tables. Nowadays weighted sum of gray gases (WSGG), ${ }^{[67]}$ spectral line based weighted sum of gray gases (SLW) ${ }^{[68]}$ and full-spectrum $k$-distribution (FSK) ${ }^{[69]}$ models are also developed. Hottel and Sarofim ${ }^{[67]}$ first proposed the WSGG model, where the non-gray gas properties are replaced by several gray gases and the heat transfer of each gray gas is calculated separately. The total heat flux is obtained afterwards by summing the heat flux of each gray gas. Smith et al. ${ }^{[70]}$ fitted the most widely used parameters of WSGG model and Modest ${ }^{[7]}$ proved that WSGG model could be used with any RTE solver. Liu et al. ${ }^{[72]}$ compared the results calculated by the simple gray gas (SGG) and WSGG models in a real natural gas fired furnace and found that WSGG performed better than SGG in the prediction of heat fluxes and gas temperature distributions. Baek et al. ${ }^{[73]}$ extended the WSGG model to the non-gray gases and gray soot particle mixtures. Results showed that the change of gas concentration significantly affected the radiation heat transfer when the particle temperature was different from the gas temperature. Subsequently, Yin et al..$^{[74]}$ refined a WSGG model with new parameters based on EWB model for oxy-fuel combustion. Considering the different partial pressure ratios of $\mathrm{H}_{2} \mathrm{O}$ and $\mathrm{CO}_{2}$, Dorigon et al. ${ }^{[75]}$ established new WSGG coefficients for pressure path-lengths changing from $0.001 \mathrm{~atm}-\mathrm{m}$ to $10 \mathrm{~atm}-$ $\mathrm{m}$ based on HITEMP 2010.

The spectral line weighted-sum-of-gray-gas (SLW) model was first developed by Denison and Webb ${ }^{[76-78]}$ and has been widely used for non-gray gas radiation heat transfer in combustion modeling. Similar to WSGG, the SLW method integrates the RTE over the gray gas rather than the wavenumber in the LBL method. Different from WSGGG, SLW uses the absorption coefficient line blackbody distribution function (ALBDF) to accurately capture the gray gas absorption coefficients and emission weighting factors for different thermal states, which is generally more accurate and flexible than the WSGG method. It is still under continuous development. Solovjov et al. ${ }^{[79,80]}$ developed the SLW-1 model, consisting of one single gray gas and also a clear gas. Good accuracy can be achieved by choosing an optimal gray gas absorption coefficient and its weight from the ALBDF. 
Table 2. Comparisons of gas radiation models.

\begin{tabular}{|c|c|c|c|c|c|c|}
\hline \multirow{2}{*}{ Model } & & \multirow{2}{*}{ Radiative property } & \multicolumn{2}{|c|}{ Homogeneous medium } & \multicolumn{2}{|c|}{ Inhomogeneous medium } \\
\hline & & & accuracy & efficiency & accuracy & efficiency \\
\hline \multirow{3}{*}{ Narrow band } & & absorption coefficient & excellent & poor & excellent & poor \\
\hline & SNB & transmissivity & excellent & general & general & general \\
\hline & SNBCK & absorption coefficient & excellent & general & general & general \\
\hline \multirow{2}{*}{ Wide band } & EWB & transmissivity & good & good & poor & good \\
\hline & WBCK & absorption coefficient & excellent & good & general & good \\
\hline \multirow{3}{*}{ Global } & WSGG & absorption coefficient & general & excellent & poor & excellent \\
\hline & SLW & absorption coefficient & excellent & excellent & good & excellent \\
\hline & FSCK & absorption coefficient & excellent & excellent & good & excellent \\
\hline
\end{tabular}

Pearson et al. ${ }^{[81,82]}$ updated ALBDF for $\mathrm{H}_{2} \mathrm{O}, \mathrm{CO}_{2}$ and $\mathrm{CO}$ using the HITEMP2010 spectroscopic database, and further derived an empirical function and tabulated data. The correlation function performs very well and the tabulated data is even better for considered various benchmark cases. Solovjov et al. ${ }^{[83]}$ developed a generalized SLW method by using ALBDF and the inverse ALBDF, which improves the efficiency in model implementation and eliminates the timeconsuming implicit calculations of the cross-sections from ALBDF for non-isotherm cases. More recently, Solovjov et $a l .{ }^{[84,85]}$ developed new kinds of correlated and scaled SLW models for non-uniform gaseous media and performed comprehensive investigations on their accuracies and provided recommendations for their applications.

In the WSGG and SLW models, the absorption coefficients are reduced to a few discrete values and the integration over the spectrum is realized by summing up the contributions of several assumptive gray gases. Based on the reordering concept, a continuous absorption coefficient distribution over the full spectrum model named FSK was proposed by Modest. ${ }^{[86]}$ Different from the narrow band and wide band models, the weights of Planck blackbody function over the full spectrum is considered in the FSK model and the solution of RTE can be converted from the wavelength space to the probability $k$-distribution space. The same accuracy of LBL can be achieved with only a little fraction of the computation time of LBL when using FSK model in homogeneous cases, ${ }^{[87]}$ as FSK model can reduce the number of times of solving RTE from the order of $10^{6}$ to $10^{1}$ without loss of accuracy when using ten points Gauss quadrature. However, the pressure, temperature and concentrations of gas species do not hold as constants but vary in practical engineering applications. To this direction, a full-spectrum correlated $k$-distribution (FSCK) model ${ }^{[86]}$ was proposed for inhomogeneous media. Wang et $a l .{ }^{[88-90]}$ constructed an FSK look-up table with four species $\mathrm{CO}_{2}, \mathrm{H}_{2} \mathrm{O}, \mathrm{CO}$ and soot and used an appropriate mixing model to get the $k$-distributions of the mixtures. Due to the overlapping effect of narrow bands of different gases, Modest and Riazzi ${ }^{[87]}$ proposed a new narrow-band mixing model based on the assumption of uncorrelatedness, thus allowing accurate assembly of mixture narrow-band and full-spectrum $k$-distributions. Based on the correlated $k$-distribution principle, Wang et al. ${ }^{[91]}$ updated the FSCK look-up table by calculating the correlated $k$-values directly from the tabulated values without the requirement of additional interpolations. Nevertheless, the amount of data increased exponentially with the number of gas species in the FSCK model. To reduce the data space of $k$-values, Zheng et al. ${ }^{[92]}$ developed a new model FSCK-RSM based on the response surface methodology (RSM). In this method, the response surface model of $k$ distributions is fitted using radial basis function and the amount of $k$-values can be reduced by 677 times for gas mixtures of $\mathrm{CO}_{2}, \mathrm{H}_{2} \mathrm{O}, \mathrm{CO}, \mathrm{C}_{2} \mathrm{H}_{2}$ and $\mathrm{C}_{2} \mathrm{H}_{4}$.

To sum up, the calculation accuracy and efficiency of gas radiative properties cannot be achieved simultaneously when various gas radiation models are used. The LBL calculation with the highest accuracy is the most computationally expensive among all the models, while WSGG with the highest efficiency cannot guarantee the calculation accuracy. FSK with its excellent computational efficiency can achieve the same high accuracy as LBL for homogeneous environments, but with large errors when dealing with inhomogeneous media. If the multi-scale FSCK is adopted to improve the calculation accuracy, the computation time will be increased as a penalty. Consequently, how to obtain the gas radiation characteristics both efficiently and accurately in any computing environment has become the focus of this field. According to the collected data from the literature, ${ }^{[31,35,61,71,86,93-}$ ${ }^{98]}$ we compare the performance of different gas radiation models in both homogeneous and inhomogeneous media in Table 2. As is shown, most models can achieve high computational accuracy for homogeneous media, with the SLW and FSCK models showing the best performance when calculation efficiency is considered as well. No radiation model can achieve the accuracy of LBL model for inhomogeneous media. The reasons are the absence of some spectral information, the gases mixing and the uncorrelated absorption coefficients for other gas radiation models in 


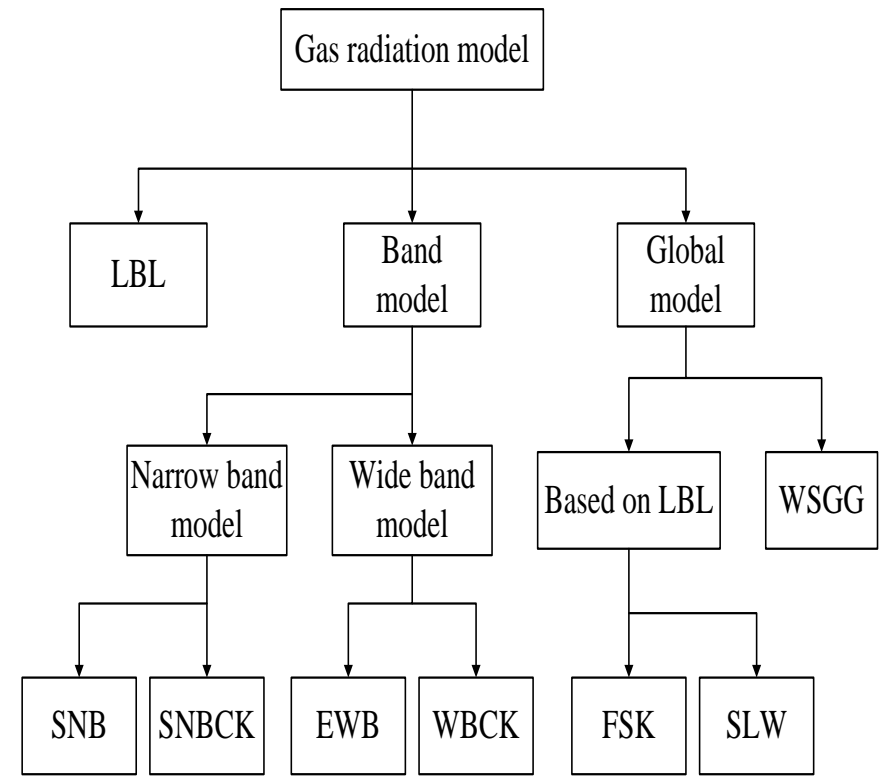

Fig. 2 The relationship of various non-gray gas radiation models.

inhomogeneous media. The hierarchical relationships of different non-gray gas radiation models are shown in Fig. 2.

There are two problems in solving radiative heat transfers of non-gray gases: (a) the establishment of non-gray gas radiation model as described above, and (b) the solutions of the RTE. It is difficult to solve the RTE because the described radiation intensity is variable in the spatial direction, and hence the RTE needs to be discretized not only in the normal but also in the angle direction. Analytical solutions of RTE can be only obtained for a few very simple objects, while in most cases only numerical solutions can be calculated. Two types of methods have been developed for solving the RTE. The first method is the ray tracing method as represented by the Zone Method, ${ }^{[99]}$ the Monte Carlo Method (MCM) ${ }^{[100-102]}$ and the Discrete Transfer Method. ${ }^{[103]}$ The second method is the global dispersion method based on differential RTE as represented by the Spherical Harmonics Method, ${ }^{[104,105]}$ the Discrete Ordinates Method (DOM) ${ }^{[106-112]}$ and the Finite Volume Method (FVM). ${ }^{[106,113-116]}$ For the first type method, an indirect solution of the RTE is obtained by calculating the radiation heat flux. For the second type method, a direct solution of the RTE is obtained via calculating the directional resolution of the radiative intensity. These methods have different characteristics in the solving process, grid requirement, computational complexity and computational accuracy. The Zone Method was first proposed by Hottel and Cohen, ${ }^{[99]}$ where the computational domain is divided into many control units called "zone" and the radiative heat flux of each unit is obtained individually. This method requires a lot of computational memory and time and thus cannot deal with problems that include scattering. $\mathrm{MCM}^{[86]}$ is always used for providing benchmark results to validate other methods. It has the advantages of simple programming, easy treatment of complex boundary conditions and good computational accuracy. However, it has several drawbacks including statistical errors, long computation time, and hence it is not suitable for engineering applications. In the Discrete Transfer Method ${ }^{[103]}$ the RTE is solved along a particular ray and it occupies less computational memory than that in MCM.

With the Spherical Harmonics Method, ${ }^{[104,105]}$ which is also known as PN approximation method, the radiation intensity is expanded by spherical harmonic function in angular space and RTE is converted into several coupled partial differential equations. The obtained differential equations are subsequently discretized and solved. In practical applications, the P1 and P3 methods are used with high frequency and only have good accuracy in the calculations of radiation heat transfer in optical thick media. The DOM was first proposed by Chandrasekhar ${ }^{[117]}$ in the study of interstellar and atmospheric radiation problems, and was then introduced by Fiveland ${ }^{[109,118]}$ and Truelove ${ }^{[119]}$ to solve the RTE. By writing the RTE in the form of each selected discrete direction, the differential equation discretized in the angular direction is solved in space by finite difference method, and then the numerical solution of radiation intensity of each space node in different angles is obtained in DOM. FVM is a development of DOM, with its basic idea discretization of the angle and space of the RTE by using finite volume method. This method was first proposed by Raithby and Chui ${ }^{[116]}$ and was applied to cartesian and cylindrical coordinate systems. Chai and Lee $^{[113]}$ used FVM to solve multidimensional radiant heat transfer problems. Compared with the DOM, the FVM discretization has the property of local conservation of element and is freer to discretize the angle. Therefore, FVM is easier to deal with irregular geometries. In addition to the aforementioned methods, others such as the Lattice Boltzmann Method (LBM), ${ }^{[120-122]}$ the Natural Element Method $(\mathrm{NEM})^{[123-125]}$ and the Meshless method ${ }^{[126,127]}$ are also under development.

In the inverse radiative transfer problems, reverse Monte Carlo (RMC) is extremely efficient to overcome the issue of normal MCM. For DOM, good results can be achieved with an adequate spatial and angular resolution, for most combustion problems, unless the geometry has a very large scale in one dimension relative to the other two. Once the gas radiation transfer is calculated, the radiation intensity in any direction that is not in the DOM quadrature scheme can be obtained in a post-process fashion by using the DOM+ISW (infinitely small weights) ${ }^{[128-130]}$ technique. For large-scale, particle-laden combustion systems, such as pulverized-coalfired furnaces, Zhou et al. ${ }^{[131,132]}$ proposed a Distributions of Ratios of Energy Scattered Or Reflected (DRESOR) method based on MCM. In this method, the distributions of energy fraction scattered by a volume element or reflected by a surface element in different directions are calculated as DRESOR values. To calculate the DRESOR values, an energy ray is tracked using the path-length method following the general procedure of the radiative energy absorption distribution method (READ). ${ }^{[133]}$ The radiative intensity at any point with high directional resolution can be obtained based 
on the DRESOR values. The DRESOR method has been successfully used to calculate radiative heat transfer in onedimensional (1D),$^{[134,135]}$ two-dimensional (2D ${ }^{[136]}$ and threedimensional (3D) ${ }^{[137]}$ systems with the particle media. Zheng et al. ${ }^{[138]}$ first combined DRESOR method with SNBCK model to study the radiative heat transfer of gas and soot mixtures in a 1D planar layer, and the SNBCK/DRESOR method showed a better efficiency than the SNBCK/DOM method when considering the wall reflection.

In Section 1, development of gas spectral line databases, gas radiation models and the RTE solution methods are reviewed in detail. In the forthcoming Sections 2 and 3, the equations of gas radiation models and the RTE solution methods will be detailed, respectively. In Section 4 , we will discuss the development of gas radiation model parameters for different gas species. In Section 5, numerical calculations are conducted for several cases using different gas radiation models and RTE solution methods in 1D, 2D and 3D systems. The accuracy and efficiency of different models in these cases will be discussed. Hopefully, this review will provide researchers with an up-to-date knowledge about the calculations of gas radiation heat transfer in combustion systems.

\section{Gas radiation models}

\subsection{LBL model}

The LBL model is the most accurate method for calculating gas radiative heat transfers, as it can provide accurate solutions when using a sufficiently accurate RTE solver and high spectral resolution. In the LBL model, the spectral absorption coefficient $\kappa_{\eta}$ is the sum of the absorption coefficients of all the overlapping spectral lines at the corresponding wavelength $\eta$, and $\kappa_{\eta}$ is expressed as ${ }^{[49]}$

$$
\kappa_{\eta}=N_{g} \sum_{i} S_{i}(T) F_{i}(\eta)
$$

where $N_{g}$ is the molecule number density of gas at the corresponding temperature and pressure, $S_{i}(T)$ the line intensity of the $i$ th spectral line at temperature $T$, and $F_{i}(\eta)$ the line shape function.

The molecule number density of gas $N_{g}$ can be obtained by

$$
N_{g}=7.3392315 \times 10^{21} \frac{P}{T}
$$

There are three kinds of line shape function $F_{i}(\eta)$ : the Lorentz line shape ${ }^{[139]}$ (collision broadening of spectral lines), the Doppler line shape ${ }^{[140]}$ (Doppler broadening effect of spectral lines at high temperature and/or low pressure) and the Voigt line shape ${ }^{[139]}$ (combined effects of collision broadening and Doppler broadening). In this paper, our discussion will be limited to the Lorentz line shape, since collision broadening usually dominates in general heat transfer applications.

The Lorentz line profile is expressed as

$$
F_{i}(\eta)=\frac{\gamma_{i}}{\pi\left[\left(\eta-\eta_{i}\right)^{2}+\gamma_{i}^{2}\right]}
$$

where $\eta_{i}$ is the center wavenumber of the $i$ th spectral line, $\gamma_{i}$ the half-width at half-maximum (HWHM) of the $i$ th spectral line, given by

$$
\gamma_{i}=\left(\frac{T_{0}}{T}\right)^{n}\left[\gamma_{\text {air }}\left(P_{0}, T_{0}\right)\left(P-P_{S}\right)+\gamma_{\text {self }}\left(P_{0}, T_{0}\right) P_{S}\right]
$$

where $P_{0}$ and $T_{0}$ are the reference pressure and temperature, respectively (for HITEMP 2010, $P_{0}=1 \mathrm{~atm}, T_{0}=296 \mathrm{~K}$ and for CDSD-1000 $P_{0}=1 \mathrm{~atm}, T_{0}=1000 \mathrm{~K}$ ). $n$ is the coefficient of the temperature dependence of the air-broadened half-width, $\gamma_{\text {air }}$ the air-broadened HWHM, $\gamma_{\text {self }}$ the self-broadened HWHM, $P$ the total pressure and $P_{S}$ the gas partial pressure.

The spectral line intensity $S_{i}(T)$ can be calculated by ${ }^{[57]}$

$$
S_{i}(T)=S_{i}\left(T_{0}\right) \exp \left(-\frac{E_{l}}{k_{B} T}+\frac{E_{l}}{k_{B} T_{0}}\right) \frac{1-\exp \left(-\frac{h c \eta_{i}}{k_{B} T}\right)}{1-\exp \left(-\frac{h c \eta_{i}}{k_{B} T_{r e f}}\right)} \frac{Q\left(T_{0}\right)}{Q(T)}
$$

where $E_{l}$ is the lower state energy, $k_{B}$ the Boltzmann constant, $h$ the Planck constant, $c$ the speed of light in vacuum and $Q$ the partition function.

When the absorption coefficient $\kappa_{\eta}$ over the whole spectrum at a certain spectral resolution is obtained using LBL as described above for given gas species concentrations and temperature distributions, the radiative intensity, the radiative source term and the heat flux can be calculated by solving the RTE.

\subsection{SNB model}

In 1957, Malkmus ${ }^{[43]}$ proposed that the distribution of spectral line intensity in a narrow band could be approximated by the exponent-tail reciprocal distribution:

$$
P(S)=\frac{1}{\ln R} S^{-1}\left[\exp \left(-\frac{S}{S_{M}}\right)-\exp \left(-\frac{R S}{S_{M}}\right)\right]
$$

where $P(S)$ is the probability density of line intensity, $R$ the ratio of the maximum spectral line intensity $S_{M}$ to the minimum spectral line intensity.

Based on the above distribution model and the Lorentz line shape function, the SNB model can give the narrow band averaged transmissivity for an isothermal homogeneous pathlength $L$ containing a radiative gas of a mole fraction $X$ and total pressure $P$, described by ${ }^{[45]}$

$$
\bar{\tau}_{\eta}(L)=\exp \left[-\frac{\pi B}{2}\left(\sqrt{1+\frac{4 S L}{\pi B}}-1\right)\right]
$$

where $B=2 \bar{\beta}_{\eta} / \pi^{2}, S=\bar{\kappa}_{\eta} X P$ and $\bar{\beta}_{\eta}=2 \pi \bar{\gamma}_{\eta} / \bar{\delta}_{\eta}$. The parameters $\bar{\kappa}_{\eta}, \bar{\gamma}_{\eta}$ and $\bar{\delta}_{\eta}$ are the average spectral line strength, the average spectral line half band-width and the average spectral line interval, respectively. They can be obtained by fitting the transmissivity results provided by the LBL model, ${ }^{[5,57]}$ and also can be directly calculated by Young's band mathematical model. ${ }^{[141]}$ It is worth noting that the HWHM of the line $\gamma_{i}$ in Eq. (4) is affected by both pressure $P$ and radiating gas concentrations $X$. Therefore, $P$ and $X$ affect the gas transmissivity and radiative heat transfer in Eq. (7) through both $B$ and $S$ in the SNB model.

Soufiani and Taine ${ }^{[56]}$ first calculated the SNB parameters $\bar{\kappa}_{\eta}, \bar{\gamma}_{\eta}$ and $\bar{\delta}_{\eta}$ based on HITRAN 1992 from 300 to $2900 \mathrm{~K}$ with a uniform bandwidth of $25 \mathrm{~cm}^{-1}$ between 150 and 9300 $\mathrm{cm}^{-1}$. $\mathrm{H}_{2} \mathrm{O}$ absorbs and emits radiation at all of the 367 narrowbands. $\mathrm{CO}_{2}$ has 96 radiating bands in the following four spectral regions: $450-1200 \mathrm{~cm}^{-1}$ (31 bands), $1950-2450 \mathrm{~cm}^{-1}$ 
(21 bands), 3300-3800 cm-1 (21 bands) and 4700-5250 $\mathrm{cm}^{-1}$ (23 bands). CO has 48 radiating bands in the following two spectral regions: $1750-2325 \mathrm{~cm}^{-1}$ (24 bands) and 3775-4350 $\mathrm{cm}^{-1}$ (24 bands). With the development of the database, Rivière and Soufiani ${ }^{[57]}$ updated the SNB parameters using HITEMP 2010 for $\mathrm{H}_{2} \mathrm{O}$ and CDSD-4000 for $\mathrm{CO}_{2}$ based on the LBL method.

Young ${ }^{[141]}$ derived new definitions for the path averages $\bar{\gamma}_{\eta}$ and $\bar{\delta}_{\eta}$ based on the traditional Curtis-Godson approximation. The new derivative models comprise more fundamental quantities than the Curtis-Godson approximation. The SNB parameters proposed by the Young's band model theory can be expressed as

$$
\begin{gathered}
\bar{\gamma}_{\eta}=\frac{1}{N} \sum_{i=1}^{N} \gamma(i) \\
\bar{\kappa}_{\eta}=\frac{1}{\Delta \eta} \sum_{i=1}^{N} S(i) \\
\bar{\delta}_{\eta}=\frac{2 \pi}{\bar{\kappa}_{\eta}}\left[\frac{1}{\Delta \eta} \sum_{i=1}^{N} \sqrt{S(i) \gamma(i)}\right]^{2}
\end{gathered}
$$

where $\Delta \eta$ is the narrow band width and $N$ the number of lines in this narrow band.

Qi et al. ${ }^{[59]}$ generated the SNB parameters for $\mathrm{C}_{2} \mathrm{H}_{4}$ and $\mathrm{C}_{2} \mathrm{H}_{2}$ using Young's band model based on HITRAN 2012 for the temperature range of 300 to $1700 \mathrm{~K}$ with an uniform bandwidth of $25 \mathrm{~cm}^{-1}$ between 150 and $9300 \mathrm{~cm}^{-1} . \mathrm{C}_{2} \mathrm{H}_{4}$ absorbs and emits radiation at all of the 367 narrow-bands. $\mathrm{C}_{2} \mathrm{H}_{2}$ has 52 radiating bands in the following two spectral regions: $675-1550 \mathrm{~cm}^{-1}$ (36 bands) and 2900-3275 $\mathrm{cm}^{-1}$ (16 bands).

Equation (7) is only applicable to homogeneous and isothermal species while the Curtis-Godson approximation method is needed for inhomogeneous or non-isothermal species. ${ }^{[142]}$ For the overlapping spectral bands of gas mixtures in the SNB model, the non-correlation hypothesis is adopted, that is, the spectral line distributions of different kinds of gases are statistically uncorrelated. In this case, the total transmissivity can be obtained by multiplying the transmissivity of each gas species proposed by Kim et al..$^{[143]}$ Since the transmissivity provided by the SNB model is not suitable for arbitrary RTE solver, the ray-tracing method ${ }^{[144]}$ is used to solve the integral RTE combined with the SNB model.

\subsection{SNBCK model}

The $k$-distribution model assumes that the Planck function is constant within a small spectral band interval. The spectral absorption coefficient is rearranged as a smooth monotonically rising curve, and the RTE is solved through the calculation of several Guass integral points.

Based on the Dirac-delta function, the probability distribution function $f(k)$ of the narrow band absorption coefficient $k$ is defined as

$$
f(k)=\frac{1}{\Delta \eta} \int_{\Delta \eta} \delta\left(k-\kappa_{\eta}\right) d \eta
$$

where $\delta\left(k-\kappa_{\eta}\right)$ is the Dirac-delta function:

$$
\delta(x)=\lim _{\delta \varepsilon \rightarrow 0}\left\{\begin{array}{l}
0,|x|>\delta \varepsilon \\
\frac{1}{2 \delta \varepsilon},|x|<\delta \varepsilon
\end{array}\right.
$$

The cumulative $k$ distribution function $g(k)$ is defined as

$$
g(k)=\int_{0}^{k} f(k) d k
$$

Hence, the mean narrow band transmissivity in homogeneous and isothermal species can be expressed as

$$
\begin{gathered}
\bar{\tau}_{\Delta \eta}(L)=\frac{1}{\Delta \eta} \int_{\Delta \eta} e^{-\kappa_{\eta} L} d \eta=\int_{0}^{\infty} e^{-k L} f(k) d k \\
=\int_{0}^{1} e^{-k(g) L} d g
\end{gathered}
$$

For inhomogeneous and non-isothermal species, the effects of temperature and pressure on the absorption coefficient must be considered. Assuming that the $k$ distribution is correlated in non-isothermal environments, relationship of the absorption coefficient and the corresponding cumulative $k$-distribution function at the same spectral position is unchanged. Thus, Eq. (14) can be rewritten as

$$
\begin{gathered}
\bar{\tau}_{\Delta \eta}(L)=\frac{1}{\Delta \eta} \int_{\Delta \eta} \exp \left(-\int_{0}^{L} \kappa_{\eta} d L\right) d \eta \\
=\int_{0}^{1} \exp \left(-\int_{0}^{L} k(L, g) d L\right) d g
\end{gathered}
$$

The SNBCK model is obtained by combining the SNB model with the associated $k$-distribution method. The gas averaged transmissivity in Eq. (7) can be expressed as the absorption coefficient distribution function $f(k)$ in the SNBCK model based on the inverse Laplace transformation: ${ }^{[61,145]}$

$$
f(k)=\frac{1}{2} k^{-3 / 2}(B S)^{1 / 2} \exp \left[\frac{\pi B}{4}\left(2-\frac{S}{k}-\frac{k}{S}\right)\right]
$$

Substituting the Eq. (16) into the Eq. (13), we can get

$$
\begin{gathered}
g(k)=\frac{1}{2}\left[1-\operatorname{erf}\left(\frac{a}{\sqrt{k}}-b \sqrt{k}\right)\right]+\frac{1}{2}\left[1-\operatorname{erf}\left(\frac{a}{\sqrt{k}}+\right.\right. \\
b \sqrt{k})] e^{\pi B}
\end{gathered}
$$

where $a=\sqrt{\pi B S} / 2, b=\sqrt{\pi B / S} / 2 . B$ and $S$ can be obtained in the SNB model and $\operatorname{erf}(x)=\frac{2}{\sqrt{\pi}} \int_{0}^{x} \exp \left(-t^{2}\right) d t$ is the error function.

After getting the $k$-distribution in the narrow band, the RTE is solved at each Gauss point and the weighted sum of the radiation intensity is obtained.

Since the information about the position of the absorption coefficient in the wavenumber-space is discarded during the generation of the $k$-distribution, any $k$-distribution model is only approximate rather than accurate when dealing with inhomogeneous and non-isothermal species. Modest and Riazzi ${ }^{[87]}$ proposed a method for calculating the $k$-distribution of mixed gases based on the assumption that the absorption coefficients of different gases are not correlated and on the transmissivity product model. ${ }^{[146]}$

Using the transmissivity product model of Taine and the product property of Laplace transform, the transmissivity of the mixed gas is calculated as

$$
\tau_{\text {mix }}=\mathscr{L}\left[f_{\text {mix }}(k)\right]=\prod_{i=1}^{N} \mathcal{L}\left[f_{i}(k)\right]
$$

For the two mixed gases, Eq. (18) can be expressed as 


$$
\begin{gathered}
\tau_{\text {mix }}=\mathscr{L}\left[f_{\text {mix }}(k)\right] \\
=\quad \int_{0}^{1} e^{-k_{1} L} d g_{1} \int_{0}^{1} e^{-k_{2} L} d g_{2} \\
=\left(\int_{g_{1}=0}^{1} \int_{g_{2}=0}^{1} e^{-\left[k_{1}\left(g_{1}\right)+k_{2}\left(g_{2}\right)\right] L} \mathrm{~d} g_{1} d g_{2}\right)
\end{gathered}
$$

To obtain an explicit expression of the cumulative distribution function $g(k)$, the integral property of the Laplace transform is used. Hence, the Laplace transform form of the $g(k)$ can be described by

$$
\begin{aligned}
\mathscr{L}\left[g_{\text {mix }}(k)\right] & =\mathscr{L}\left[\int_{0}^{k} f_{\text {mix }}(k) d k\right] \\
& =\left(\int_{g_{1}=0}^{1} \int_{g_{2}=0}^{1} e^{-\left[k_{1}\left(g_{1}\right)+k_{2}\left(g_{2}\right)\right] L} \mathrm{~d} g_{1} d g_{2}\right) \frac{1}{L} \\
& =\left(\int_{g_{1}=0}^{1} \int_{g_{2}=0}^{1} \frac{e^{-\left[k_{1}\left(g_{1}\right)+k_{2}\left(g_{2}\right)\right] L}}{L} \mathrm{~d} g_{1} d g_{2}\right)
\end{aligned}
$$

Using the inverse Laplace transform, we can get

$$
\begin{aligned}
g_{\text {mix }}\left(k_{\text {mix }}\right) & =\int_{g_{1}=0}^{1} \int_{g_{2}=0}^{1} H\left[k_{\text {mix }}-\left(k_{1}+k_{2}\right)\right] \mathrm{d} g_{1} d g_{2} \\
& =\int_{g_{1}=0}^{1} g_{2}\left(k_{\text {mix }}-k_{1}\right) d g_{1}
\end{aligned}
$$

Extending the number of gas species to more than two, Eq. (21) can be rewritten as

$$
\begin{aligned}
& g_{\text {mix }}\left(k_{\text {mix }}\right)=\int_{g_{1}=0}^{1} \cdots \int_{g_{N}=0}^{1} H\left[k_{\text {mix }}-\sum_{i=1}^{N} k_{i}\right] \\
& \mathrm{d} g_{1} \cdots d g_{N}
\end{aligned}
$$

Modest and Riazzi ${ }^{[87]}$ proved that the results calculated via Eq. (22) are much more accurate than that by $g\left(k_{\text {mix }} \mathrm{O}\left(\prod_{i=1}^{N} g_{i}\left(k_{i}\right)\right)_{\text {mix }}{ }^{[89]}\right.$ for $\mathrm{H}_{2} \mathrm{O}$ and $\mathrm{CO}_{2}$ gas mixtures.

\subsection{WBCK model}

The SNBCK model extracts the gas absorption coefficient from the transmissivity in the SNB model, which overcomes the difficulties of the SNB model. As a result, the SNBCK model can be used in any RTE solver, especially the efficient and accurate DOM. Since the bandwidth in the SNBCK model is $25 \mathrm{~cm}^{-1}$ that results in low computational efficiency, Liu et $a l .{ }^{[64]}$ developed the WBCK model by using a band lumping strategy to improve the efficiency of the SNBCK model. In the WBCK model, band lumping aggregates several (usually 5-20) contiguous narrow bands into a wide band. Instead of solving the RTE multiple times for each narrow band in the wideband, the radiative transfer over the wideband is calculated by concentrating several successive narrow bands into a single wideband. In general, the wider the lumped band, the higher the calculation efficiency, but at the cost of greater errors because the blackbody function cannot be treated as constant.

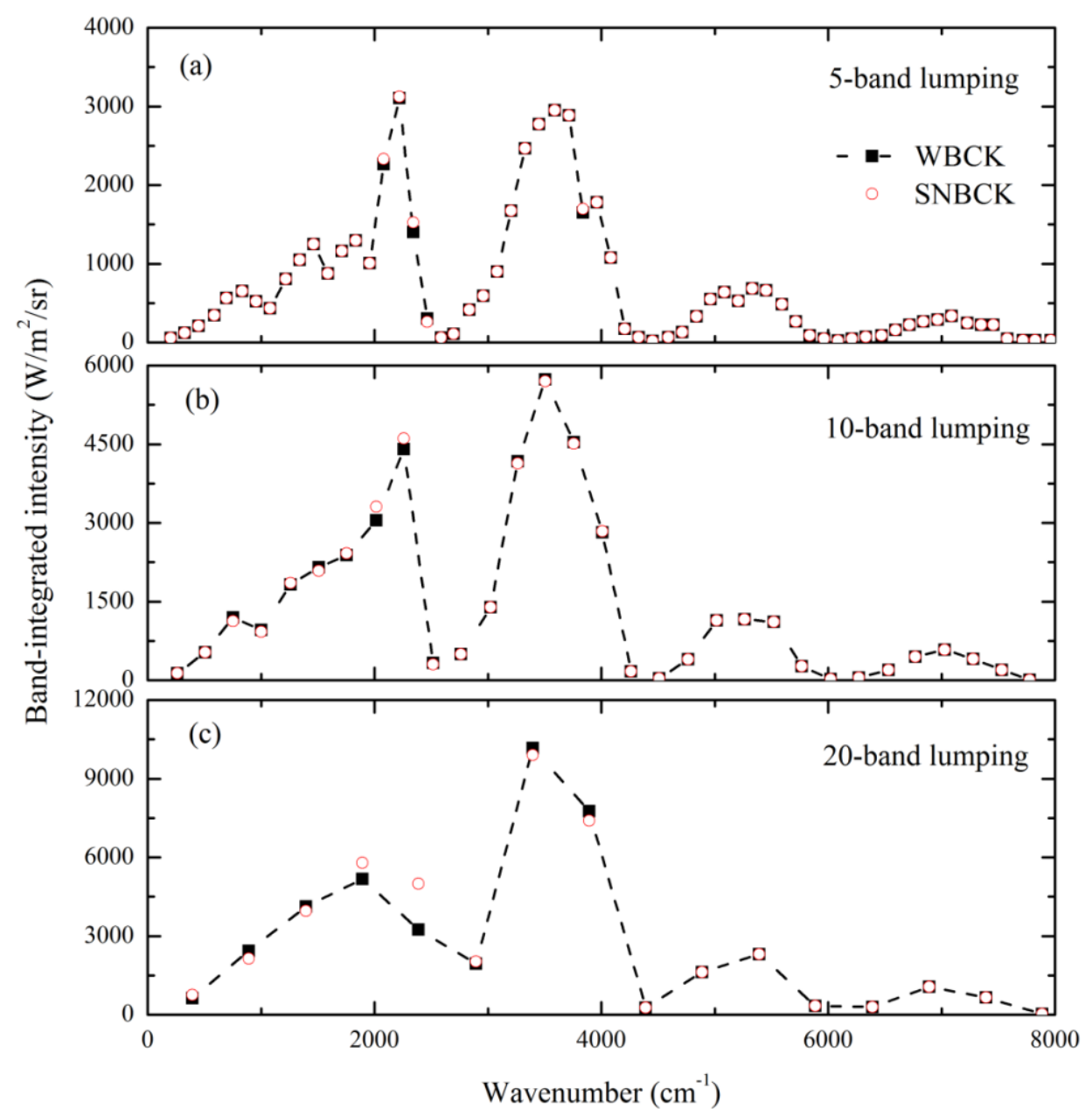

Fig. 3 Comparisons of band-integrated intensities calculated by the SNBCK and WBCK models: (a) $125 \mathrm{~cm}^{-1}$ bandwidth, (b) 250 $\mathrm{cm}^{-1}$ bandwidth and (c) $500 \mathrm{~cm}^{-1}$ bandwidth. (Reproduced with the permission form [64]. Copyright@American Institute of Aeronautics and Astronautics.) 
The mean gas transmissivity of $M$ successive narrow bands in a wide band is calculated as

$$
\bar{\tau}_{w b}=\frac{1}{M} \sum_{i=1}^{M} \bar{\tau}_{i}
$$

where $\bar{\tau}_{i}$ is the $i$ th mean narrow band transmissivity in the wide band calculated by the SNB model.

For narrow bands with different widths, the wideband gas transmissivity is the bandwidth weighted average of the gas transmissivity within the narrow band, rather than the simple algebraic average. According to the inverse Laplace transformation, the absorption coefficient distribution function in the wideband can be described as

$$
f_{w b}(k)=\frac{1}{M} \sum_{i=1}^{M} f_{i}(k)
$$

where the absorption coefficient distribution function $f_{i}(k)$ at each narrow band is calculated by the SNBCK model.

Subsequently, the cumulative $k$ distribution function at wideband $g_{w b}(k)$ is calculated as

$$
g_{w b}(k)=\frac{1}{M} \sum_{i=1}^{M} g_{i}(k)
$$

Lacis and Oinas ${ }^{[60]}$ and Liu et al..$^{[61,97]}$ proved that the gas absorption coefficient at the wideband could be obtained from the inversion of the above equation using the NewtonRaphson iteration method. To compare the results calculated by the SNBCK and WBCK models, Liu et al. ${ }^{[64]}$ carried out numerical calculations in a $1 \mathrm{D}$ planar layer for band-integrated radiation intensities. The parallel-plane layer was filled with $\mathrm{CO}_{2}, \mathrm{H}_{2} \mathrm{O}$ and $\mathrm{N}_{2}$, and was considered as a non-isothermal inhomogeneous medium. The thickness of the layer was $1.0 \mathrm{~m}$ and the temperature of the two walls was $300 \mathrm{~K}$ and nonemitting. The temperature of the gas mixture was given as $T=$ $1000+1400 \times 4 \times x / L-1400 \times 4 \times(x / L)^{2}$. The mole fraction of $\mathrm{CO}_{2}$ was $Y_{\mathrm{CO}_{2}}=0.05+0.1 \times 4 \times x / L-0.1 \times$ $4 \times(x / L)^{2}$, and the mole fraction of $\mathrm{H}_{2} \mathrm{O}$ was $Y_{\mathrm{H}_{2} \mathrm{O}}=0.6-$ $0.4 \times 4 \times x / L+0.4 \times 4 \times(x / L)^{2}$. The calculation space was divided into 80 uniform volumes. Fig. 3 shows the bandintegrated intensities calculated by the SNBCK and WBCK models with 5-band lumping, 10-band lumping and 20-band lumping. It can be seen that the WBCK predictions (5-band and 10-band lumping) are in excellent agreement with the

SNBCK predictions, while the errors of band-integrated intensity calculated by the 20-band lumping are larger than other cases. The large difference in Fig. 3(c) at $2387.5 \mathrm{~cm}^{-1}$ is due to the intense radiation and banded behavior of $\mathrm{CO}_{2}$ in the $4.3 \mu \mathrm{m}$ band. Obviously, the narrower bands are lumped, the less accurate the results are obtained with the WBCK model.

\subsection{WSGG model}

The principle of the WSGG model is to simulate the nongray radiation characteristics of real gases by adding a transparent gas to several gray gases as shown in Fig. 4. ${ }^{[14]}$ The parameters of classical WSGG models are obtained by fitting emissivity calculated by the LBL or SNB models. The accuracy of fitting is related to the accuracy of the benchmark models. To improve the accuracy and to broaden the general working conditions for the WSGG model, the correlation formula of the absorption coefficient changing with gas partial pressure ratio is obtained by fitting. The total emissivity of the gas can be weighted by $N$ gray gases, ${ }^{[67]}$

$$
\varepsilon=\sum_{i=0}^{N} a_{i}(T)\left(1-e^{-k_{i} P X L}\right)
$$

where $a_{i}$ is the weighting factor of the $i$ th gray gas when the absorption coefficient is $k_{i}$.

The weighting factor $a_{i}$ is considered as a polynomial function of temperature of order $J,^{[148]}$

$$
a_{i}=\sum_{j=1}^{J} b_{i, j} T^{j-1}
$$

where the coefficients $b_{i, j}$ are set as constants in the early WSGG models. Jonhansson et al..$^{[149]}$ fitted $b_{i, j}$ into a more accurate polynomial function of the $\mathrm{H}_{2} \mathrm{O}$-to- $\mathrm{CO}_{2}$ partial pressure ratio, and $T$ in Eq. (27) was replaced by $T / T_{\text {ref }}$ based on the normalized temperature $T_{r e f}=1200 \mathrm{~K}$.

$b_{i, j}$ in Eq. (27) is expressed as

$$
b_{i, j}=b 1_{i, j}+b 2_{i, j} \frac{P_{\mathrm{H}_{2} \mathrm{O}}}{P_{\mathrm{CO}_{2}}}+b 3_{i, j}\left(\frac{P_{\mathrm{H}_{2} \mathrm{O}}}{P_{\mathrm{CO}_{2}}}\right)^{2}
$$

Considering the "transparent window" effect in real gases, a transparent gas is introduced into the WSGG model $\left(k_{0}=0\right)$ and the weighting factor of the transparent gas is $a_{0}=1-$ $\sum_{i=0}^{N} a_{i}$.

The gray gas absorption coefficient is calculated by
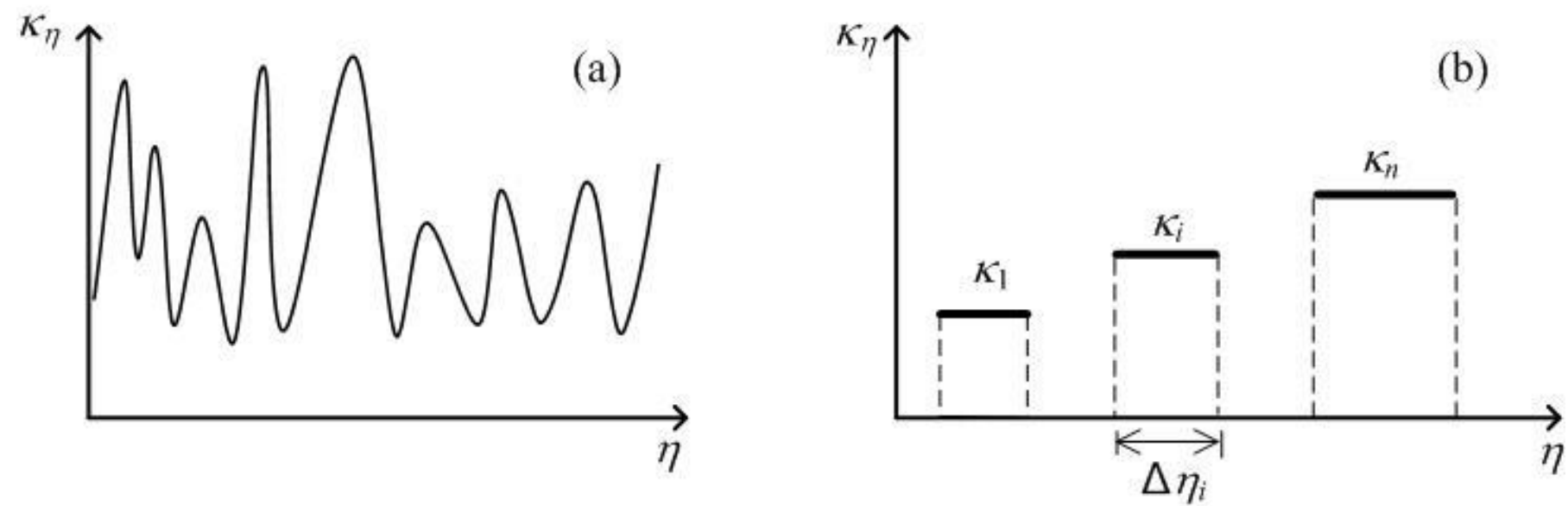

Fig. 4 (a) High-resolution absorption coefficient distribution of a real gas; (b) a representation of the WSGG model. (Reproduced with the permission from [147]. Copyright@2017 Chinese Physical Society.) 


$$
k_{i}=k 1_{i}+k 2_{i} \frac{P_{\mathrm{H}_{2} \mathrm{O}}}{\mathrm{P}_{\mathrm{CO}_{2}}}
$$

The RTE is solved for each gray gas after getting the absorption coefficient and weighting factor. Afterwards, the total heat flux can be obtained by using weighted sum of the heat flux of each gray gas. Since the absorption coefficients of the gases can be obtained directly from the WSGG model, it can be easily combined with the solution method of RTE in any form and thus the calculation efficiency is high.

\subsection{SLW model}

The SLW model is a particular case of the global model and an improved version of the WSGG method. In this method, the entire wavenumber range is portioned into many intervals by a set of supplemental cross-sections $\tilde{C}_{0}, \tilde{C}_{1}, \ldots, \tilde{C}_{n}$, which are logarithmically spaced between the minimum and maximum values. Within each interval, the mean absorption cross section is evaluated as

$$
C_{j}=\sqrt{\tilde{C}_{j-1} \tilde{C}_{j}}
$$

The spectral integration of the RTE in the SLW model needs the so-called F function the Absorption Line Blackbody Distribution Function (ALBDF),

$$
F\left(C, T \int_{\left\{\eta: C_{\eta}<C\right\}}^{\int d} E_{b \eta}\left(T_{b}\right) / E_{b}\left(T_{b} b_{g}\right.\right.
$$

Which denotes the fraction of the blackbody emissive power for the spectrum intervals where the cross-sections are smaller than $C$.

The spectral integration of the RTE yields the following equation for an absorbing and emitting medium (without scattering),

$$
\frac{d I_{J}}{d s}=-k_{j} I_{j}+a_{j} k_{j} I_{b}
$$

where $\kappa_{j}$ is the gray gas absorption coefficient, whose calculated from the ALBDF evaluated for the $j$-th crosssection interval,

$$
\begin{gathered}
a_{j}=F\left(\tilde{C}_{j}, T_{g}, T_{b}\right)-F\left(\tilde{C}_{j-1}, T_{g}, T_{b}\right) \\
a_{0}=F\left(C_{\text {min }}, T_{g}, T_{b}\right)
\end{gathered}
$$

These weights should be considered in the emissive powers of both gas elements and wall surface elements.

After solving the RTE for all representative gray gases in each interval, the total intensity can be calculated as the sum of all gray gas intensities,

$$
I=\sum_{j=0}^{n} I_{j}
$$

According to its definition, the ALBDF is a function of the absorption cross section and the given conditions of the medium (temperature, pressure, concentrations). It can be either calculated from a high-resolution spectroscopic database or a correlation. Pearson and $\mathrm{Webb}^{[82]}$ have recently updated the hyperbolic tangent correlations for $\mathrm{CO}_{2}, \mathrm{H}_{2} \mathrm{O}$ and CO using the HITEMP2010 database.

\subsection{FSK model}

The FSK model was proposed by Modest ${ }^{[86]}$ based on the reordering concept. In this model, the gas absorption coefficients are rearranged in a numerical order. A new artificial spectrum is constructed according to the frequency of the absorption coefficients in the spectral range, and then the distribution function of the absorption coefficient in the artificial spectrum is obtained. At the same time, the weight of Planck blackbody function over the full spectrum is considered.

For a homogeneous medium, the full-spectrum Planckfunction weighted $k$-distribution is expressed as

$$
f(T, k)=\frac{1}{I_{b}(T)} \int_{0}^{\infty} I_{b \eta}(T) \delta\left(k-\kappa_{\eta}\right) d \eta
$$

where $\delta(x)$ is the Dirac-delta function.

The reordering RTE can be obtained by multiplying the Dirac-delta function on both sides of the RTE and integrating over the full spectrum,

$$
\begin{aligned}
\frac{d I_{k}}{d s}= & k f(T, k) I_{b}-\left(k+\sigma_{s}\right) I_{k}+ \\
& \frac{\sigma_{s}}{4 \pi} \int_{4 \pi} I_{k}\left(\hat{s}^{\prime}\right) \Phi_{\eta}\left(\hat{s^{\prime}}, \hat{s}^{\prime}\right) d \Omega^{\prime}
\end{aligned}
$$

The boundary condition is

$$
\begin{aligned}
& I_{k}=I_{w k}=\varepsilon_{w} f\left(T_{w}, k\right) I_{b w}+ \\
& \left(1-\varepsilon_{w}\right) \frac{1}{\pi} \int_{\hat{n} \cdot \hat{s}<0} I_{k}|\hat{n} \cdot \hat{s}| d \Omega
\end{aligned}
$$

where $I_{k}=\int_{0}^{\infty} I_{\eta} \delta\left(k-\kappa_{\eta}\right) d \eta$ is the sum of the spectral radiation intensity when $k=\kappa_{\eta}$.

Equations (37) and (38) can be rewritten via dividing both sides by

$$
\begin{gathered}
f(T, k) \frac{d I_{g}}{d s}=k\left(I_{b}(T)-I_{g}\right)-\sigma_{s} \\
\left(I_{g}-\frac{1}{4 \pi} \int_{4 \pi} I_{g}\left(\hat{s}^{\prime}\right) \Phi_{\eta}\left(\hat{s^{\prime}}, \hat{s}^{\prime}\right) d \Omega^{\prime}\right) \\
I_{g}=I_{w g}=\varepsilon_{w} a\left(T_{w}, T, g\right) I_{b w}+ \\
\left(1-\varepsilon_{w}\right) \frac{1}{\pi} \int_{\hat{n} \cdot \hat{s}<0} I_{g}|\hat{n} \cdot \hat{s}| d \Omega
\end{gathered}
$$

where $\quad I_{g}=\frac{I_{k}}{f(T, k)}=\frac{\int_{0}^{\infty} I_{\eta} \delta\left(k-\kappa_{\eta}\right) d \eta}{f(T, k)} \quad$ and $\quad a\left(T_{w}, T, g\right)=$ $\frac{f\left(T_{w}, k\right)}{f(T, k)}=\frac{d g_{w}\left(T_{w}, k\right)}{d g(T, k)}$.

After a series of transformations, the RTE is finally transformed from the spectral space to the $g$ space. Consequently, the total radiation intensity can be obtained by

$$
I=\int_{0}^{\infty} I_{\eta} d \eta=\int_{0}^{1} I_{g} d g
$$

For an inhomogeneous medium, a thermodynamic state vector $\varphi$ is introduced in the FSK model, which contains all the parameters that affect the spectral absorption coefficient such as temperature, pressure and concentration. It is also assumed that the spectral absorption coefficients at any wavelength are correlated to each other in different physical states, i.e. $\kappa_{\eta}(\eta, \varphi)=k_{\eta}^{*}\left(\varphi, k_{\eta}\right)$.

Therefore, the full-spectrum $k$-distribution function and cumulative $k$-distribution in an inhomogeneous medium can be described as ${ }^{[86]}$

$$
\begin{gathered}
f(T, \varphi, k)=\frac{1}{I_{b}(T)} \int_{0}^{\infty} I_{b \eta}(T) \delta\left(k-\kappa_{\eta}(\varphi, \eta)\right) d \eta \\
g(T, \varphi, k)=\int_{0}^{k} f(T, \varphi, k) d k
\end{gathered}
$$


The temperature $T$ in the $f(T, \varphi, k)$ and $g(T, \varphi, k)$ is the Planck temperature and $\varphi$ represents the state of the corresponding value of $k$. The cumulative $k$-distribution function depends only on the Planck temperature based on the FSK model, that means:

$$
\begin{aligned}
g(T, \varphi, k) & =\int_{0}^{k} f(T, \varphi, k) d k=\int_{0}^{k^{*}} f\left(T, \varphi^{*}, k^{*}\right) d k^{*} \\
& =g\left(T, \varphi^{*}, k^{*}\right)
\end{aligned}
$$

The above formula shows that no matter what $k$ value is, the cumulative $k$-distribution is in the same $g$ space, as long as the temperature of the Planck function is the same.

In an inhomogeneous medium, it is necessary to determine an unified $g$ space for spectral integration of the RTE due to the change of state parameters. The Planck temperature and status vector in the unified reference $g$ space are usually called reference temperature $T_{r e f}$ and reference state $\varphi_{\text {ref }}$. Hence, Eq. (33) can be converted to the RTE in FSCK for an inhomogeneous medium

$$
\begin{gathered}
\frac{d I_{g}}{d s}=k^{*}\left(T_{r e f}, \varphi, g_{r e f}\right)\left[a\left(T, T_{r e f}, g_{r e f}\right) I_{b}(T)-I_{g}\right]- \\
\sigma_{s}\left(I_{g}-\frac{1}{4 \pi} \int_{4 \pi} I_{g}\left(\hat{s}^{\prime}\right) \Phi_{\eta}\left(\hat{s}, \hat{s}^{\prime}\right) d \Omega^{\prime}\right)
\end{gathered}
$$

where $a\left(T, T_{r e f}, g_{r e f}\right)=d g\left(T, \varphi_{r e f}, k\right) / d g\left(T_{r e f}, \varphi_{r e f}, k\right)$.

The $k$-distributions in the FSCK can be retrieved directly from the tabulated values, avoiding multiple computations and interpolation and thus saving considerable CPU time during the radiation calculation. Nevertheless, the radiation properties of gas mixtures still occupy a large amount of data space, whose size increases exponentially with the number of gas species in the FSCK model. A new model FSCK-RSM has been proposed by Shu et al. ${ }^{[22]}$ based on the response surface methodology (RSM). The $k$-distributions of FSCK is used to fit the response surface model based on the radial basis function and the radiative calculation efficiency is improved by avoiding multiple computations and interpolation in the FSCK-RSM.

To improve the fitting characteristics of the near-linear region, some additional polynomials and constraint conditions are adopted for FSCK-RSM in the radial basis function (RBF): ${ }^{[150]}$

$$
\begin{aligned}
\hat{f}(\boldsymbol{x})= & \sum_{j=1}^{M} \lambda_{j} \varphi\left(\left\|\boldsymbol{x}-\boldsymbol{x}^{(j)}\right\|\right)+\sum_{i=1}^{N} b_{i} P_{i}(\boldsymbol{x}) \\
& \sum_{j=1}^{M} P_{i}\left(\boldsymbol{x}^{(j)}\right) \cdot \lambda_{j}=0
\end{aligned}
$$

where $\hat{f}(\boldsymbol{x})$ is the predicted absorption coefficient, $x=$ $\left\{x_{1}, x_{2}, \ldots, x_{l}\right\}$ the vector of variables (reference temperature, local temperature and gas concentration), $l$ the number of variables, $\left\|\mathbf{x}-\mathbf{x}^{(j)}\right\|$ the Euclidean distance between an arbitrary state $\mathbf{x}$ and a sampling state $\mathbf{x}^{(j)}, M$ the number of sampling states, $\lambda=\left\{\lambda_{1}, \lambda_{2}, \ldots, \lambda_{M}\right\}$ the regression coefficient vector of radial basis function, $b=\left\{b_{1}, b_{2}, \ldots, b_{N}\right\}$ the regression coefficient of the additional polynomials, $N=\frac{1}{n !} \prod_{i=1}^{n}(l+i)$ the number of mutually orthogonal polynomial terms and $n$ the degree of polynomial.
The Latin hypercubes design method ${ }^{[151]}$ can be applied to obtain the global properties of the actual response problem through a minimum number of sampling states. Subsequently, $\lambda=\left\{\lambda_{1}, \lambda_{2}, \ldots, \lambda_{M}\right\}$ and $b=\left\{b_{1}, b_{2}, \ldots, b_{N}\right\}$ can be obtained by solving Eqs. (46) and (47). The fitting is evaluated by the R-Squared $\left(R^{2}\right)$ and normalized maximal relative errors $\left(e_{\max }\right)$. The FSCK-RSM can be seen as a machine learning method to predict the relation between the input and output of complex systems, which is exactly how to calculate the radiation characteristics of gas mixtures from spectral line data of spectral database by FSCK. Machine learning is a field of computer science that gives computer systems the ability to find relations between inputs and outputs and to learn from experiences without actually modeling the physical and chemical laws that govern the system. ${ }^{[152]}$ It is foreseeable that machine learning will be the main developed direction of FSCK model in the future.

Both SLW and FSK are global methods which treats the spectral integration over the full spectrum by only several gray gases. The difference between these two methods is that the SLW performs the spectral integration over discrete cross sections, while the FSK integrates over the Planck-weighted function (g) by Gaussian quadrature. This leads to different numerical treatments, especially for the gas absorption coefficient calculation. The ALBDF in the SLW method is used to get the $\mathrm{F}$ function value from the given cross-section (equivalent to absorption coefficient) value, and the FSK function in the FSK is used to obtain the absorption coefficient from given $\mathrm{g}$. The ALBDF is indeed the inverse function to the FSK function.

Despite the difference in numerical implementations of the FSK and SLW, they all originate from the same radiative transfer equation. Solovjov and Webb ${ }^{[153]}$ have demonstrated that as the number of gray gases in the SLW approaches infinity, the convectional SLW method yields the exact solution for the spectral integration of the RTE in isothermal and uniform media like the FSK method. They also proved that SLW and FSK are related and can be mutual transferred.

\section{RTE solution methods}

The RTE for the intensity field in an absorbing, emitting, and scattering gray enclosure with vacuum is ${ }^{[86]}$

$$
\begin{gathered}
\hat{s} \cdot \nabla I_{\eta}(r, \hat{s})=\kappa_{\eta} I_{b \eta}(r, \hat{s})-\beta_{\eta} I_{\eta}(r, \hat{s}) \\
+\frac{\sigma_{s \eta}}{4 \pi} \int_{4 \pi} I_{\eta}\left(r, \hat{s}_{i}\right) \Phi_{\eta}\left(\hat{s}_{i}, \hat{s}\right) d \Omega_{i}
\end{gathered}
$$

The source function $S_{\eta}\left(r^{\prime}, \hat{s}\right)$ is described by

$$
\begin{gathered}
S_{\eta}\left(r^{\prime}, \hat{s}\right)=\left(1-\omega_{\eta}\right) I_{b \eta}\left(r^{\prime}\right)+ \\
\frac{\omega_{\eta}}{4 \pi} \int_{4 \pi} I_{\eta}\left(r^{\prime}, \hat{s}_{i}\right) \Phi_{\eta}\left(\hat{s}_{i}, \hat{s}\right) d \Omega_{i}
\end{gathered}
$$

The boundary condition at any surface is written as

$$
I_{w \eta}\left(r_{w}, \hat{s}\right)=\varepsilon\left(r_{w}\right) I_{b \eta}\left(r_{w}\right)+\int_{\hat{n}, \hat{s}^{\prime}<0} \rho\left(r_{w}, \hat{s}^{\prime}, \hat{s}\right) I_{\eta}\left(r_{w}, \hat{s}^{\prime}\right) \mid \hat{n} \cdot
$$




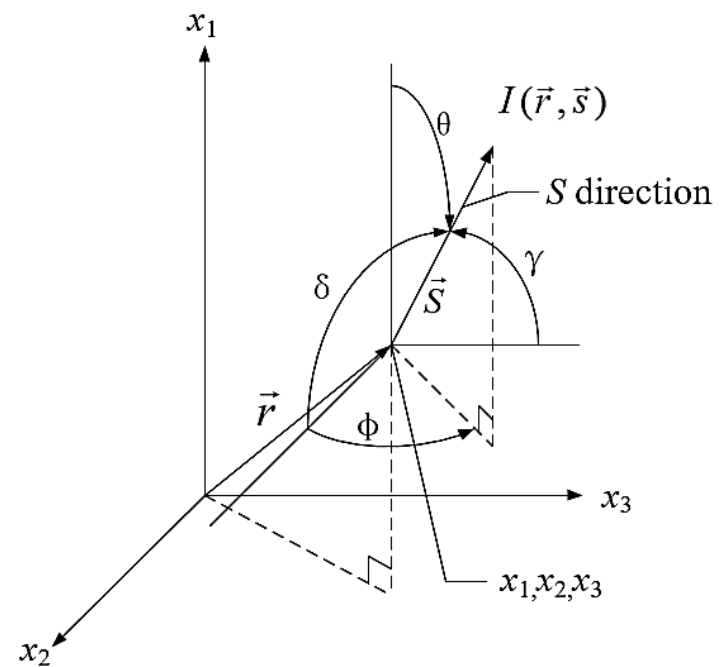

Fig. 5 Coordinate system and radiation intensity in the PN model. (Reproduced with the permission from [33]. Copyright@2021 CRC Press.)

\subsection{PN method}

If the radiation medium is assumed to be gray, the absorption and scattering coefficients do not change with space and the scattering is isotropic, the RTE can be expressed as

$$
\frac{d I}{d s}=\kappa I_{b}-\left(\kappa+\sigma_{s}\right) I+\frac{\sigma_{s}}{4 \pi} \int_{\Omega=0}^{4 \pi} I(s, \Omega) d \Omega
$$

Fig. 5 shows a schematic diagram of the radiation intensity in any direction at any spatial position in the Cartesian coordinate system. The radiation intensity in the $s$ direction can be expressed as a function of polar angle $\theta$ and azimuthal angle $\phi$, or direction cosine $l_{i}(i=1,2,3)$,

$$
\begin{gathered}
\frac{d I}{d s}=\cos \theta \frac{\partial I}{\partial x_{1}}+\sin \theta \cos \varphi \frac{\partial I}{\partial x_{2}}+\sin \theta \sin \varphi \frac{\partial I}{\partial x_{3}} \\
=l_{1} \frac{\partial I}{\partial x_{1}}+l_{2} \frac{\partial I}{\partial x_{2}}+l_{3} \frac{\partial I}{\partial x_{3}}
\end{gathered}
$$

where $l_{1}=\cos \theta, l_{2}=\cos \delta, l_{3}=\cos \gamma$.

When using optical thickness coordinates $d \tau_{i}=\left(\kappa+\sigma_{s}\right) d x_{i}$ and scattering albedo $\omega$, Eq. (51) can be rewritten as

$$
\sum_{i=1}^{3} l_{i} \frac{\partial I}{\partial \tau_{i}}+I=(1-\omega) I_{b}+\frac{\omega}{4 \pi} \int_{\Omega=0}^{4 \pi} I(s, \Omega) d \Omega
$$

In the PN method, the radiation intensity in any direction $s$ is expressed as the series of orthogonal spherical harmonic functions:

$$
I(s, \Omega)=\sum_{l=0}^{\infty} \sum_{m=-l}^{l} A_{l}^{m}(s) Y_{l}^{m}(\Omega)
$$

The spherical harmonic function approximation method is accurate when $l \rightarrow \infty . A_{l}^{m}(S)$ are the coefficients related to the spatial position and $Y_{l}^{m}(\Omega)$ are the normalized spherical harmonic functions related to the angular direction,

$$
Y_{l}^{m}(\Omega)=\left[\frac{2 l+1}{4 \pi} \frac{(l-|m|) !}{(l+|m|) !}\right]^{1 / 2} e^{j m \phi} P_{l}^{|m|}(\cos \theta)
$$

where $j=\sqrt{-1}, P_{l}^{|m|}(\cos \theta)$ is the first type of associated Legendre polynomials with the degree of freedom $l$ and the order $m$

$P_{l}^{|m|}(\mu)=\frac{\left(1-\mu^{2}\right)^{|m| / 2}}{2^{l} l !} \frac{d^{l-|m|}}{d \mu^{l-|m|}}\left(\mu^{2}-1\right)^{l}$

where $\mu=\cos \theta, P_{l}^{|m|}(\mu)=0$ when $|m|>l, P_{l}^{0}(\mu)=$ $P_{l}(\mu)$.

When applying the PN method, it is necessary to truncate the spherical harmonic function series of the above radiation intensity. In the radiation heat transfer calculations, generally $l=0, \quad 1 \quad$ (P1 approximation) or $l=0,1,2,3 \quad$ (P3 approximation) is used. Theoretically, other higher-order terms can be retained, while P3 approximation is sufficient for general engineering calculations. The accuracy of the evenorder approximations ( $\mathrm{P} 2, \mathrm{P} 4$, etc.) is slightly improved compared with the odd-order approximations of the lower first order. As it is difficult to apply boundary conditions, the oddorder approximations are used in actual applications.

In order to calculate the radiation intensity, $A_{l}^{m}(S)$ in the series must be calculated first. This can be obtained from the moment equation, derived by multiplying the local radiation intensity by the direction cosines (either alone or in combination) and subsequently integrating them in angular space. For example, the zero order, first order, second order and general order are as follows

$$
\begin{aligned}
& I^{(0)}(s)=\int_{\Omega=0}^{4 \pi} I(s, \Omega) d \Omega \\
& I^{(i)}(s)=\int_{\Omega=0}^{4 \pi} l_{i} I(s, \Omega) d \Omega \\
& I^{(i j)}(s)=\int_{\Omega=0}^{4 \pi} l_{i} l_{j} I(s, \Omega) d \Omega \\
& I^{(i j k l \ldots)}(s)=\int_{\Omega=0}^{4 \pi} l_{i} l_{j} l_{k} l_{l} \ldots I(s, \Omega) d \Omega
\end{aligned} \quad(i=1,2,3)
$$

The zero-moment divided by the light speed is the radiation energy density. The first moment is the radiation energy flow rate in the corresponding direction. The second moment divided by the light speed is the tensors of local stress and pressure. Moments of higher order have no practical physical significance.

Substituting the spherical harmonic function of radiation intensity into the moment equation, a specific model can be obtained after truncating certain terms and integrating them. The P3 method contains 20 coupled algebraic equations in 20 radiation intensity moments. With the P3 approximation method, the radiation intensity can be expressed as the corresponding moment,

$$
\begin{aligned}
& 4 \pi I(s, \theta, \varphi)=I^{(0)}+3 I^{(1)} \cos \theta+3 I^{(2)} \sin \theta \cos \varphi+3 I^{(3)} \sin \theta+\frac{5}{4}\left(3 I^{(11)}-I^{(0)}\right)\left(3 \cos ^{2} \theta-1\right)+15\left(I^{(12)} \cos \varphi+\right. \\
& \left.\left.I^{(13)} \sin \varphi\right) \cos \theta \sin \theta+\frac{15}{4}\left[\left(I^{(22)}-I^{(33)}\right) \cos 2 \varphi+2 I^{(23)}\right) \sin 2 \varphi\right] \sin ^{2} \theta+\frac{7}{4}\left(5 I^{(111)}-3 I^{(1)}\right)\left(5 \cos ^{3} \theta-3 \cos \theta+\right. \\
& \frac{21}{8}\left[\left(5 I^{(211)}-I^{(2)}\right) \cos \varphi+\left(5 I^{(311)}-I^{(3)} \sin \varphi\right)\right]\left(5 \cos ^{2} \theta-1\right) \sin \theta+\frac{105}{4}\left[\left(I^{(122)}-I^{(133)}\right) \cos 2 \varphi+\right. \\
& \left.\left.\left.2 I^{(123)} \sin 2 \varphi\right)\right] \cos \theta+\frac{35}{8}\left[\left(I^{(222)}-3 I^{(233)}\right) \cos 3 \varphi-\left(I^{(333)}-3 I^{(322)}\right) \sin 3 \varphi\right)\right] \sin ^{3} \theta
\end{aligned}
$$


along with the identities,

$$
\begin{gathered}
I^{(0)}=I^{(11)}+I^{(22)}+I^{(33)} \\
I^{(1)}=I^{(111)}+I^{(222)}+I^{(333)} \\
I^{(2)}=I^{(211)}+I^{(222)}+I^{(233)} \\
I^{(3)}=I^{(311)}+I^{(322)}+I^{(333)}
\end{gathered}
$$

The P1 model is much simpler and only includes the first four items of Eq. (58)

$$
\begin{gathered}
4 \pi I(s, \theta, \varphi)=I^{(0)}+3 I^{(1)} \cos \theta+3 I^{(2)} \sin \theta \cos \varphi+ \\
3 I^{(3)} \sin \theta \sin \varphi
\end{gathered}
$$

Therefore, the P1 method is much simpler than the P3 method and thus more widely used.

To make the equation closed, an expression of these moments is also needed, which can be obtained by solving the corresponding moment equation in the RTE. The original RTE, namely the zero-order moment equation, can also be written as follows:

$$
\sum_{i=1}^{3} l_{i} \frac{\partial I}{\partial \tau_{i}}+I=(1-\omega) I_{b}+\frac{\omega}{4 \pi} I^{(0)}
$$

Multipling the above equation by the single direction cosine or multiple direction cosines and integrating in the angle space, the moment equation for the P3 method is obtained,

$$
\begin{gathered}
\sum_{i=1}^{3} \frac{\partial I^{(i)}}{\partial \tau_{i}}=(1-\omega)\left(4 \pi I_{b}-I^{(0)}\right) \\
\sum_{i=1}^{3} \frac{\partial I^{(i j)}}{\partial \tau_{i}}=-I^{(j)}(3 \text { equations: } j=1,2,3) \\
\sum_{i=1}^{3} \frac{\partial I^{(i j k)}}{\partial \tau_{i}}=-I^{(j k)}+\frac{4 \pi}{3} \delta_{j k}\left[(1-\omega) I_{b}+\right. \\
\left.\frac{\omega}{4 \pi} I^{(0)}\right](9 \text { equations: } j, k=1,2,3) \\
\sum_{i=1}^{3} \frac{\partial I^{(i j k l)}}{\partial \tau_{i}}=-I^{(j k l)}(27 \text { equations: } j, k, l=1,2,3)
\end{gathered}
$$

where $\delta_{j k}$ is Kronecker operator.

For the P1 method, only the first two equations above are included. $I^{(i j k l)}$ in P3 method and $I^{(i j)}$ in P1 method need to be approximated because they are unknown quantities, which can be obtained by substituting the truncated radiation intensity expression into the definition of moment,

$$
\begin{gathered}
I^{(i j k l)}=\frac{1}{7}\left(I^{(i j)} \delta_{k l}+I^{(i k)} \delta_{j l}+I^{(i l)} \delta_{i l}+I^{(k l)} \delta_{j k}+\right. \\
\left.I^{(j l)} \delta_{i k}\right)-\frac{1}{35}\left(\delta_{i l} \delta_{k l}+\delta_{i l} \delta_{j k}+\delta_{i k} \delta_{j l}\right) \\
I^{(i j)}=\frac{1}{3} \delta_{i j} I^{(0)}
\end{gathered}
$$

Consequently, $I^{(11)}=I^{(22)}=I^{(33)}=I^{(0)} / 3$ and $I^{(i j)}=0$.

For an opaque gray wall with a wall emissivity of $\varepsilon$, the outward radiation intensity consists of emission and reflection parts,

$$
I_{o}(\Omega)=\varepsilon I_{b, w}+\frac{1}{\pi} \int_{\Omega_{i}=0}^{2 \pi} \rho I\left(\Omega_{i}\right) l_{i} d \Omega_{i}
$$

where $l_{i}$ is the cosine between the direction of the radiation intensity and the normal direction of the wall.

The general Marshak boundary condition is
$\int_{\Omega=0}^{2 \pi} I_{o}(\Omega) Y_{l}^{m}(\Omega) d \Omega=\int_{\Omega=0}^{2 \pi}\left[\varepsilon I_{b, w}+\right.$

$\left.\frac{1}{\pi} \int_{\Omega_{i}=0}^{2 \pi} \rho I\left(\Omega_{i}\right) l_{i} d \Omega_{i}\right] d \Omega$

\subsection{DOM}

In the DOM, the angle space is discretized into $n$ portions, and there is a partial differential equation in each angle direction. The integral at the angle can be replaced by a numerical integral, namely

$$
\int_{4 \pi} f(\hat{\boldsymbol{s}}) d \Omega \approx \sum_{i=1}^{n} w_{i} f\left(\hat{\boldsymbol{s}}_{i}\right)
$$

where $w_{i}$ is the integral weight in the $\hat{\boldsymbol{s}}_{i}$ direction.

Then the RTE can be rewritten as

$$
\hat{\boldsymbol{s}}_{i} \cdot \nabla I_{i}=k I_{b}-\beta I_{i}+\frac{\sigma_{s}}{4 \pi} \sum_{j=1}^{n} w_{j} I_{j} \Phi, \quad i=1,2, \ldots, n
$$

with the boundary condition:

$$
I_{w, i}=\varepsilon I_{b w}+\frac{\rho}{\pi} \sum_{\widehat{\boldsymbol{n}} \cdot \hat{\boldsymbol{s}}_{j}<0} w_{j} I_{w, j}\left|\widehat{\boldsymbol{n}} \cdot \hat{\boldsymbol{s}}_{j}\right|, \widehat{\boldsymbol{n}} \cdot \hat{\boldsymbol{s}}_{\boldsymbol{i}}>0
$$

Each ray in the $s$ direction intersects the boundary of the region twice: once it is emitted from the wall $\left(\widehat{\boldsymbol{n}} \cdot \hat{\boldsymbol{s}}_{i}>0\right)$; the other reaches the wall and is absorbed or reflected $\left(\widehat{\boldsymbol{n}} \cdot \hat{\boldsymbol{s}}_{i}<\right.$ 0 ). The first order equation of this equation requires only one boundary condition. A total of $n$ coupled systems of first order linear partial differential equations can be solved by analytical or numerical methods. If there is scattering in the calculation, all equations are coupled regardless of whether the wall is reflecting or not, and generally multiple iterative steps are needed to obtain the solution. If the calculation problem is radiation-equilibrium and the temperature distribution is unknown, an iterative solution is required even when there is no scattering or wall reflection. When the temperature distribution is known and scattering and wall reflectivity are not taken into account, each direction only needs to be iterated once to obtain the result.

After getting the radiation intensity, the values of the integral variables can be solved by integrating the values in all directions. The radiative heat flux in the medium or on the wall is

$$
\boldsymbol{q}_{r}=\int_{4 \pi} I \hat{\boldsymbol{s}} d \Omega \approx \sum_{i=1}^{n} w_{i} I_{i} \hat{\boldsymbol{s}}_{i}
$$

Incident radiative flux $G$ is calculated according to the following formula:

$$
G=\int_{4 \pi} I d \Omega=\sum_{i=1}^{n} w_{i} I_{i}
$$

The choice of integral method is arbitrary, but generally there are some restrictions for the discretization of the direction and weights. Popular methods include S2, S4, S6 and S8, among which any direction is

$$
\hat{\boldsymbol{s}}_{i}=\xi_{i} \hat{\boldsymbol{\imath}}+\eta_{i} \hat{\boldsymbol{\jmath}}+\mu_{i} \widehat{\boldsymbol{k}}
$$

Table 3 lists the values when the direction cosine is positive, covering one-eighth of the angular space. 
Table 3. Direction cosine and weight coefficients in the DOM. ${ }^{[86]}$

\begin{tabular}{|c|c|c|c|c|}
\hline $\begin{array}{l}\text { Discrete } \\
\text { method }\end{array}$ & $\begin{array}{l}\nabla(k \nabla T) \\
=\nabla \cdot q_{r}\end{array}$ & $\boldsymbol{k}$ & $\begin{array}{l}\nabla \cdot q_{r} \\
=\kappa\left(4 \sigma T^{4}\right. \\
-G)\end{array}$ & $\boldsymbol{\kappa}$ \\
\hline \multirow[t]{2}{*}{$\mathrm{S}_{2}$} & 0.5773503 & 0.5773503 & 0.5773503 & 1.5707963 \\
\hline & 0.2958759 & 0.2958759 & 0.9082483 & 0.5235987 \\
\hline \multirow[t]{5}{*}{$\mathrm{S}_{4}$} & 0.2958759 & 0.9082483 & 0.2958759 & 0.5235987 \\
\hline & 0.9082483 & 0.2958759 & 0.2958759 & 0.5235987 \\
\hline & 0.1838670 & 0.1838670 & 0.9656013 & 0.1609517 \\
\hline & 0.1838670 & 0.6950514 & 0.6950514 & 0.3626469 \\
\hline & 0.1838670 & 0.9656013 & 0.1838670 & 0.1609517 \\
\hline \multirow{8}{*}{$\mathrm{S}_{6}$} & 0.6950514 & 0.1838670 & 0.6950514 & 0.3626469 \\
\hline & 0.6950514 & 0.6950514 & 0.1838670 & 0.3626469 \\
\hline & 0.9656013 & 0.1838670 & 0.1838670 & 0.1609517 \\
\hline & 0.1422555 & 0.1422555 & 0.9795543 & 0.1712359 \\
\hline & 0.1422555 & 0.5773503 & 0.8040087 & 0.0992284 \\
\hline & 0.1422555 & 0.8040087 & 0.5773503 & 0.0992284 \\
\hline & 0.1422555 & 0.9795543 & 0.1422555 & 0.1712359 \\
\hline & 0.5773503 & 0.1422555 & 0.8040087 & 0.0992284 \\
\hline \multirow{5}{*}{$\mathrm{S}_{8}$} & 0.5773503 & 0.5773503 & 0.5773503 & 0.4617179 \\
\hline & 0.5773503 & 0.5773503 & 0.1422555 & 0.0992284 \\
\hline & 0.8040087 & 0.1422555 & 0.5773503 & 0.0992284 \\
\hline & 0.8040087 & 0.5773503 & 0.1422555 & 0.0992284 \\
\hline & 0.9795543 & 0.1422555 & 0.1422555 & 0.1712359 \\
\hline
\end{tabular}

According to the discrete direction above, the RTE in the Cartesian coordinate system can be expressed as

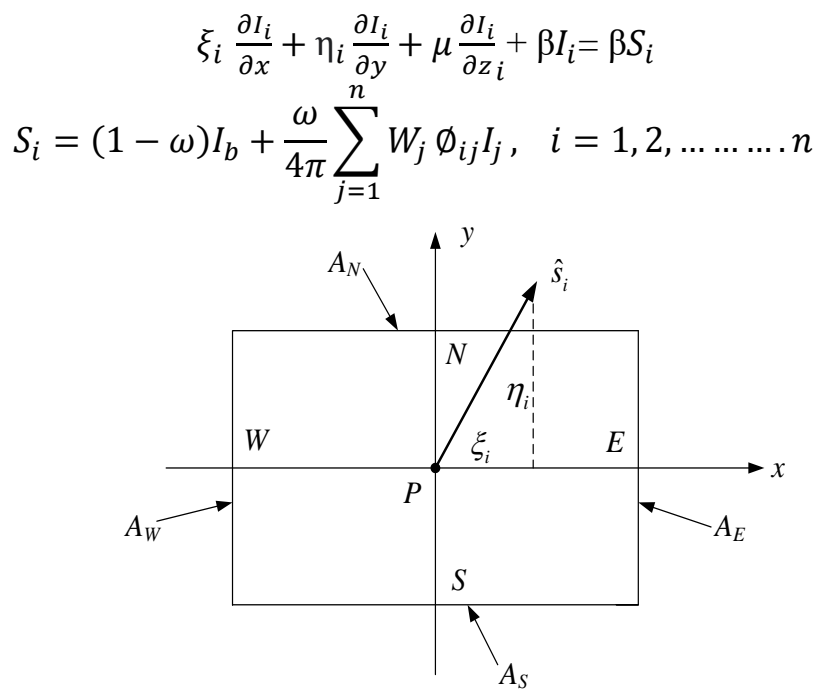

Fig. 6 Two dimensional control volume in DOM. (Reproduced with the permission from [86]. Copyright@2013 Elsevier Ltd.)

For a $2 \mathrm{D}$ region, there is no gradient in the $z$ direction. Finite volume method is adopted to calculate the above equations. The control volume is shown in Fig. 6. Integrating Eq. (76) in this unit, one can get

$\xi_{i}\left(A_{E} I_{E i}-A_{W} I_{W i}\right)+\eta_{i}\left(A_{N} I_{N i}-A_{S} I_{s i}\right)=-\beta I_{P i}+\beta V S_{P i}$ (78) where $\tau=5, \mathrm{Nc}=0.01$ are the mean values in the unit.

As there are too many unknown parameters in the above equation, which need to be reduced by associating the center and boundary values. The following equation is usually used

$$
I_{p i}=\gamma_{y} I_{N i}+\left(1-\gamma_{y}\right) I_{S i}=\gamma_{x} I_{E i}+\left(1-\gamma_{x}\right) I_{W i}
$$

where $\gamma_{x}, \gamma_{y}$ are constants in the range of 0.5 to 1 . When the value is 0.5 , it is a diamond scheme; when the value is 1 , it is a first-order upwind format or step scheme. When the calculation starts at the bottom left in Fig. 6, both $\xi_{i}$ and $\eta_{i}$ are positive.

By substituting Eq. (79) into Eq. (78), we can get

$$
I_{p i}=\frac{\beta V S_{p i}+\xi_{i} A_{E W} I_{W i} / \gamma_{x}+\eta_{i} A_{N S} I_{S i} / \gamma_{y}}{\beta V+\xi_{i} A_{E} / \gamma_{x}+\eta_{i} A_{N} / \gamma_{y}}
$$

where $A_{E W}=\left(1-\gamma_{x}\right) A_{E}+\gamma_{x} A_{W}$ and $A_{N S}=\left(1-\gamma_{y}\right) A_{N}+$ $\gamma_{y} A_{S}$

After getting $I_{p i}$ of each node, the radiation intensity of the right interface and upper interface of the element can be obtained according to the above equations. The same calculation needs to be repeated three times from the other corners when the first quadrant cycle is over.

If the wall is black and without scattering, the radiation intensity of each node in each direction needs to be calculated only once. If there are reflections on the wall or the medium is scatterable, multiple iterations are required. After each iteration, the radiation intensity of the wall and the radiation source term in the medium are updated and subsequently recalculated until the radiation intensity is converged.

\subsection{FVM}

The DOM cannot guarantee the conservation of radiant energy because of the simple numerical integration in angular space, even though the finite volume method is used in spatial discretization. The principle of FVM is to use the finite volume method in both Cartesian and angular coordinates. Therefore, the conservation of radiant energy is guaranteed and the moment and half moment can be accurately calculated in any volume.

Fig. 7 shows the spatial unit and solid angle unit distribution after the two dimensional spatial discretization. Integrating RTE in space element and solid angle, it is able to get

$$
\begin{gathered}
\int_{\Omega_{i}} \int_{V} \frac{\partial I}{\partial s} d V d \Omega=\int_{\Omega_{i}} \int_{V}\left(\kappa I_{b}-\beta I\right) d V d \Omega+ \\
\int_{\Omega_{i}} \int_{V} \frac{\sigma_{s}}{4 \pi} \int_{4 \pi} \Phi\left(\hat{\boldsymbol{s}}^{\prime}, \hat{\boldsymbol{s}}\right) d \Omega^{\prime} d V d \Omega
\end{gathered}
$$

The left side can be simplified to

$$
\int_{\Omega_{i}} \int_{V} \frac{\partial I}{\partial s} d V d \Omega=\int_{\Omega_{i}} I \boldsymbol{s} \cdot \boldsymbol{n} d \Gamma d \Omega
$$

where $\Gamma$ is the boundary of the control volume.

Assuming that the radiation intensity is constant at the element interface and the solid angle, Eq. (81) can be rewritten as 

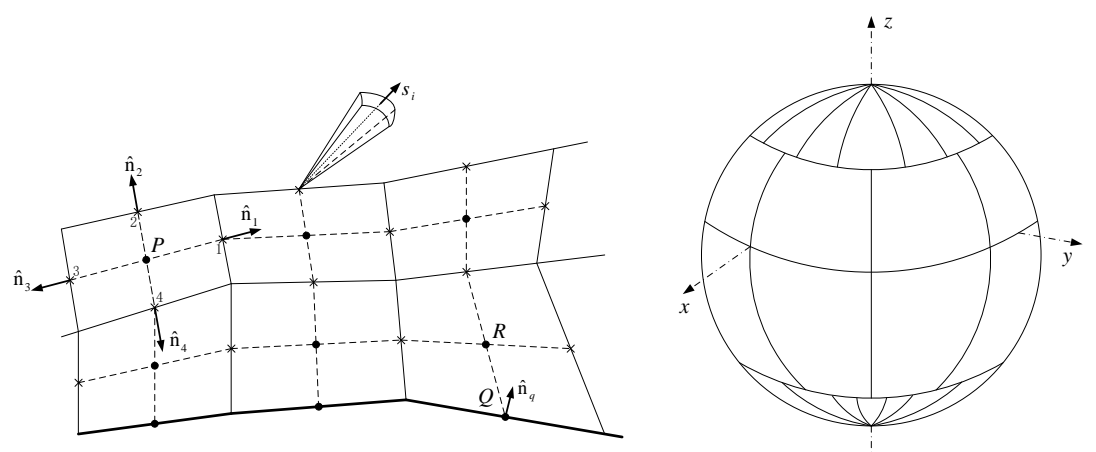

Fig. 7 Spatial and angular discretization in FVM. (Reproduced with the permission from [86]. Copyright@2013 Elsevier Ltd.)

$$
\begin{gathered}
\sum_{k} I_{k i}\left(\boldsymbol{s}_{i} \cdot \boldsymbol{n}_{k}\right) A_{k}=\beta_{p}\left(S_{p i}-I_{p i}\right) V \Omega_{i}, \\
S_{p i}=\left(1-\omega_{p}\right) I_{b p}+\frac{\omega_{p}}{4 \pi} \sum_{j=1}^{n} I_{p j} \bar{\Phi}_{i j}, \\
\bar{\Phi}_{i j}=\frac{1}{\Omega_{i}} \int_{\Omega_{i}} \int_{\Omega_{j}} \bar{\Phi}\left(\boldsymbol{s}^{\prime}, \boldsymbol{s}\right) d \Omega^{\prime} d \Omega, \\
\boldsymbol{s}_{i}=\int_{\Omega_{i}} \boldsymbol{s} d \Omega
\end{gathered}
$$

where subscripts $k, p$ represent the center of the unit interface $A_{k}$ and the unit center point.

Adopting the step scheme, Eq. (83) can be transformed into

$$
I_{p i}=\frac{\beta_{p} S_{p i} V \Omega_{i}+\sum_{k, \text { in }} I_{k i}\left|s_{i} \cdot \boldsymbol{n}_{k}\right| A_{k}}{\beta_{p} V \Omega_{i}+\sum_{k, \text { out }}\left|\boldsymbol{s}_{i} \cdot \boldsymbol{n}_{k}\right| A_{k}}
$$

with the boundary condition

$$
I_{w}=\varepsilon I_{b w}+(1-\varepsilon) \frac{\sum_{i, \text { in }} I_{w i}\left|s_{i} \cdot n_{w}\right|}{\sum_{i, \text { out }}\left|s_{i} \cdot n_{w}\right|}
$$

When the radiation intensity in all directions is solved, the incident radiation and heat flux in the medium can be calculated by the following formula:

$$
G_{p}=\sum_{i} I_{p i} \Omega_{i}, \quad q_{r p}=\sum_{i} I_{p i} s_{i}
$$

\subsection{DRESOR method}

In standard DOM, we can only get the radiation intensity in a relatively small number of directions, which are prescribed in the angular quadrature scheme used in the calculation. Also the DOM+ISW technique is available and can be easily used to obtain radiation intensity along any direction by postprocessing. In the PN method, even the directional resolution of intensity can be higher, the truncation of the orthogonal series causes errors. MCM can deal with complex cases and take into account all the important effects in the radiation heat transfer simulations without making an approximation. The accuracy of MCM is determined by the tradeoff between the computational time and the statistical error. ${ }^{[154]}$ However, the MCM can only provide precious information of radiative heat exchange between different grids of the computing object, while a high directional resolution of the radiation intensity is not MCM's advantage. The DRESOR method was proposed by Zhou et al. ${ }^{[135,155]}$ based on MCM, in which the angular distributions of intensity at one or more points or even anywhere in the medium, can be calculated without the need of radiation equilibrium according to the given temperature distribution and radiative characteristics in the medium. ${ }^{[132]}$

In the DRESOR method, the source function $S_{\eta}\left(r^{\prime}, \hat{s}\right)$ in Eq. (49) is rewritten as

$$
\begin{gathered}
S_{\eta}\left(r^{\prime}, s\right)=\frac{1}{4 \pi \beta_{\eta}}\left[4 \pi\left(1-\omega_{\eta}\right) \beta_{\eta} I_{b \eta}\left(r^{\prime}\right)+\right. \\
\left.\beta_{\eta} \omega_{\eta} \int_{4 \pi} I_{\eta}\left(r^{\prime}, s_{i}\right) \Phi_{\eta}\left(s_{i}, s\right) d \Omega_{i}\right]=\frac{1}{4 \pi \beta_{\eta}}\left[Q_{e \eta}\left(r^{\prime}\right)+f_{1}\left(r^{\prime}, s\right)\right]
\end{gathered}
$$

where $Q_{e \eta}\left(r^{\prime}\right)=4 \pi \beta_{\eta}\left(1-\omega_{\eta}\right) I_{b \eta}\left(r^{\prime}\right)$ and $f_{1}\left(r^{\prime}, s\right)=$ $\sigma_{s \eta} \int_{4 \pi} I_{\eta}\left(r^{\prime}, s_{i}\right) \Phi_{\eta}\left(s_{i}, s\right) d \Omega_{i}$. The energy scattered by the medium, $f_{1}\left(r^{\prime}, s\right)$, is the source of the indirect contribution to the radiation intensity. ${ }^{[156]}$ Its contribution to the radiation intensity can be treated in the same way as the energy emitted by $Q_{e \eta}\left(r^{\prime}\right)$.

The boundary condition in Eq. (50) is rewritten as

$$
\begin{aligned}
I_{w \eta}\left(r_{w}, s\right) & =\varepsilon\left(r_{w}\right) I_{b \eta}\left(r_{w}\right)+\frac{1}{\pi} f_{2}\left(r_{w}, s\right) \\
& =\frac{1}{\pi}\left[Q_{e \eta}\left(r_{w}\right)+f_{2}\left(r_{w}, s\right)\right]
\end{aligned}
$$

where $\frac{1}{\pi} f_{2}\left(r_{w}, s\right)=\int_{n \cdot s /<0} \rho^{\prime \prime}\left(r_{w}, s^{\prime}, s\right) I_{\eta}\left(r_{w}, s^{\prime}\right)|n \cdot s| d \Omega^{\prime}$, $Q_{e \eta}\left(r_{w}\right)=\pi \varepsilon\left(r_{w}\right) I_{b \eta}\left(r_{w}\right)$. The contribution of the reflected energy $f_{2}\left(r_{w}, s\right)$ to the radiation intensity after being projected onto the surface can be treated in the same way as the energy emitted by the wall $Q_{e \eta}\left(r_{w}\right)$.

Substituting Eqs. (87) and (88) into the integral form of the Eq. (48) yields

$I_{\eta}(r, s)=\frac{1}{\pi}\left[\pi \varepsilon\left(r_{w}\right) I_{b \eta}\left(r_{w}\right)+f_{2}\left(r_{w}, s\right)\right] \exp \left[-\int_{0}^{S} \beta_{\eta} d s^{\prime \prime}\right]+$
$\int_{0}^{S} \frac{1}{4 \pi}\left[4 \pi \beta_{\eta}\left(1-\omega_{\eta}\right) I_{b \eta}\left(r^{\prime}\right)+f_{1}\left(r^{\prime}, s\right)\right] \exp \left[-\int_{0}^{S^{\prime}} \beta_{\eta} d s^{\prime \prime}\right] d s^{\prime}$

where $I_{b \eta}\left(r_{w}\right)$ and $I_{b \eta}\left(r^{\prime}\right)$ are the direct parts in the total intensity $I_{\eta}(r, \hat{s})$.

In the DRESOR method, $f_{1}\left(r^{\prime}, s\right)$ and $f_{2}\left(r_{w}, s\right)$ are the indirect parts, namely $R_{d}^{S}$, and the DRESOR values are used for a quantitative description of the integral parts in Eqs. (49) and (50).

Therefore, the equation for $I_{\eta}(r, \hat{s})$ by the DRESOR method is described as 


$$
\begin{aligned}
& I_{\eta}(r, \hat{s})=\frac{1}{\pi}\left\{\pi \varepsilon\left(r_{w}\right) I_{b \eta}\left(r_{w}\right)+\int_{W} R_{d}^{s}\left(r_{w}{ }^{\prime}, r_{w}, \hat{s}\right)\left[\pi \varepsilon\left(r_{w}{ }^{\prime}\right) I_{b \eta}\left(r_{w}{ }^{\prime}\right)\right] d A^{\prime}\right. \\
& \left.+\int_{V} R_{d}^{s}\left(r^{\prime \prime}, r_{w}, \hat{s}\right)\left[4 \pi \beta_{\eta}\left(1-\omega_{\eta}\right) I_{b \eta}\left(r^{\prime \prime}\right)\right] d V^{\prime \prime}\right\} \exp \left[-\int_{0}^{s} \beta_{\eta} d s^{\prime \prime}\right] \\
& +\int_{0}^{s} \frac{1}{4 \pi}\left\{4 \pi \beta_{\eta}\left(1-\omega_{\eta}\right) I_{b \eta}\left(r^{\prime}\right)+\int_{W} R_{d}^{s}\left(r_{w}{ }^{\prime}, r^{\prime}, \hat{s}\right)\left[\pi \varepsilon\left(r_{w}{ }^{\prime}\right) I_{b \eta}\left(r_{w}{ }^{\prime}\right)\right] d A^{\prime}\right. \\
& \left.\quad+\int_{V} R_{d}^{s}\left(r^{\prime \prime}, r^{\prime}, \hat{s}\right)\left[4 \pi \beta_{\eta}\left(1-\omega_{\eta}\right) I_{b \eta}\left(r^{\prime \prime}\right)\right] d V^{\prime \prime}\right\} \exp \left[-\int_{0}^{s^{\prime}} \beta_{\eta} d s^{\prime \prime}\right] d s^{\prime}
\end{aligned}
$$
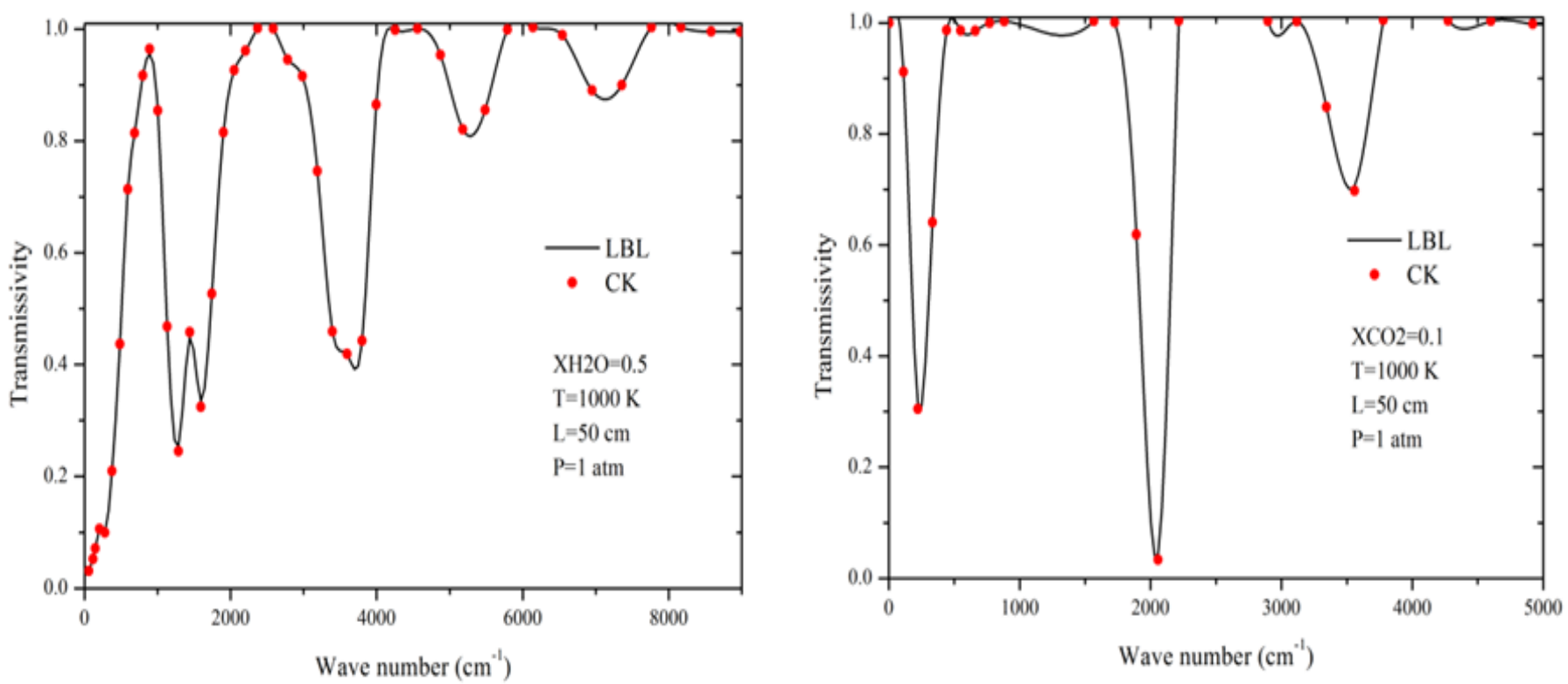

Fig. 8 Transmissivity of an isothermal and homogeneous column of $\mathrm{H}_{2} \mathrm{O} / \mathrm{N}_{2}$ and $\mathrm{CO}_{2} / \mathrm{N}_{2}$ mixtures at variable spectral resolution (the same as for CK parameters). (Reproduced with the permission form [56]. Copyright@1996 Elsevier Ltd.)

where $R_{d}^{S}\left(r_{w}{ }^{\prime}, r_{w}, \hat{s}\right), R_{d}^{S}\left(r^{\prime \prime}, r_{w}, \hat{s}\right), R_{d}^{S}\left(r_{w}{ }^{\prime}, r^{\prime}, \hat{s}\right)$ and $R_{d}^{s}\left(r^{\prime \prime}, r^{\prime}, \hat{s}\right)$ are the ratios of the energy scattered or reflected by a unit area or volume around the point of the second vector $\left(r_{w}\right.$ and $\left.r^{\prime}\right)$ into an unit solid angle around the direction $\hat{s}$ to that emitted from an unit area or volume around the point of the first vector $\left(r_{w}{ }^{\prime}\right.$ and $\left.r^{\prime \prime}\right)$ multiplied by $4 \pi$.

For the given distributions of $\kappa(r), \sigma_{s}(r), \varepsilon\left(r_{w}\right)$ and $\rho\left(r_{w}, \hat{s}^{\prime}, \hat{s}\right)$, if the DRESOR values including $R_{d}^{s}\left(r_{w}{ }^{\prime}, r_{w}, \hat{s}\right)$, $R_{d}^{S}\left(r^{\prime \prime}, r_{w}, \hat{s}\right), R_{d}^{S}\left(r_{w}{ }^{\prime}, r^{\prime}, \hat{s}\right)$ and $R_{d}^{S}\left(r^{\prime \prime}, r^{\prime}, \hat{s}\right)$ are known, the angular distribution of radiation intensity $I_{\eta}(r, \hat{s})$ can be described by a function of the $I_{b \eta}\left(r^{\prime}\right)$ medium radiation and the $I_{b \eta}\left(r_{w}{ }^{\prime}\right)$ surface radiation. The DRESOR values can be calculated by the path-length method, which tracks every ray in the procedure. ${ }^{[131]}$

\section{Gas radiation model parameters}

In this section, we discuss the development of gas radiation model parameters for different gas species.

\subsection{NB model parameters}

Soufiani and Taine ${ }^{[56]}$ first presented the narrow band parameters of $\mathrm{H}_{2} \mathrm{O}, \mathrm{CO}_{2}$ and $\mathrm{CO}$ at a spectral interval $25 \mathrm{~cm}^{-1}$ from 150 to $9300 \mathrm{~cm}^{-1}$ at temperatures up to $2500 \mathrm{~K}$. The parameters of $\mathrm{H}_{2} \mathrm{O}^{[157]}$ and $\mathrm{CO}_{2}{ }^{[158]}$ were generated from the
HITRAN 92 and the data of Flaud et al. ${ }^{[159]}$ for $\mathrm{H}_{2} \mathrm{O}$ near 2.7 $\mu \mathrm{m}$. The spectroscopic data for CO were from Refs. ${ }^{[160,161]}$ The CK parameters were generated with a varying spectral interval, so that the relative change in spectral equilibrium intensity could be kept within $4 \%$ in the whole temperature range. Transmissivity of an isothermal and homogeneous medium was calculated by the SNB and CK models and compared with LBL. Fig. 8 compares the narrow-band transmissivities calculated using the $\mathrm{CK}$ and LBL models and the difference of the two model results for $\mathrm{H}_{2} \mathrm{O}\left(X_{\mathrm{H} 2 \mathrm{O}}=0.5\right)$ and $\mathrm{CO}_{2}$ $\left(X_{\mathrm{CO} 2}=0.1\right)$ at $T=1000 \mathrm{~K}$. Under uniform conditions, the SNB and CK methods have good agreement in the whole spectrum.

With the development of spectroscopic databases, Rivière and Soufiani ${ }^{[57]}$ updated the band model parameters for $\mathrm{H}_{2} \mathrm{O}$, $\mathrm{CO}_{2}, \mathrm{CO}$ and $\mathrm{CH}_{4}$ at high temperatures. The results calculated by the new parameters were compared with those of the LBL, and with those of the earlier model parameters ${ }^{[56]}$ in terms of band averaged spectral transmissivities, Planck mean absorption coefficients, and total emissivities. The HITEMP 2010 database ${ }^{[29]}$ was selected for calculating the $\mathrm{H}_{2} \mathrm{O}$ and $\mathrm{CO}$ absorption spectra. For generating the detailed absorption spectrum of $\mathrm{H}_{2} \mathrm{O}$ in the LBL solution, a progressive line wing cutoff was used: $500 \mathrm{~cm}^{-1}$ for temperatures below $2100 \mathrm{~K}, 20$ $\mathrm{cm}^{-1}$ in the temperature range $2100-3300 \mathrm{~K}, 100 \mathrm{~cm}^{-1}$ for temperatures above $3300 \mathrm{~K} .500 \mathrm{~cm}^{-1}$ was used as line wing 

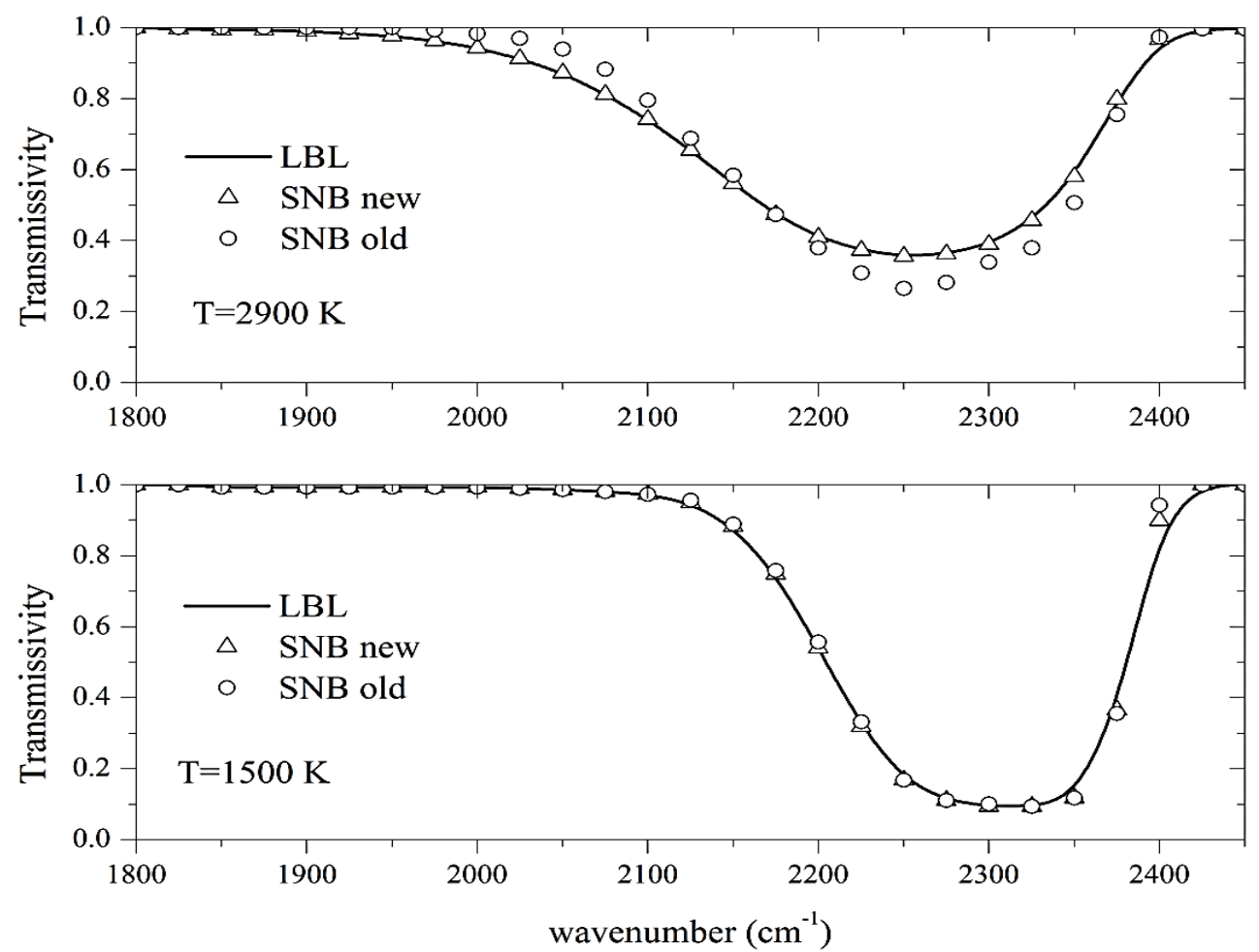

Fig. $9 \mathrm{CO}_{2}$ transmissivity in the $4.3 \mu \mathrm{m}$ region at $1500 \mathrm{~K}$ and $2900 \mathrm{~K} . X_{\mathrm{CO} 2}=0.1, L=10 \mathrm{~cm}, \mathrm{P}=1 \mathrm{~atm}$. (Reproduced with the permission form [57]. Copyright@2012 Elsevier Ltd.)

cutoff for CO. The CDSD-4000 database ${ }^{[23]}$ and the line wing cutoff $50 \mathrm{~cm}^{-1}$ were used for $\mathrm{CO}_{2}$. The database provided by Perrin and Soufiani ${ }^{[162]}$ was selected for calculating the $\mathrm{CH}_{4}$ absorption spectra, which were obtained from statistical extensions under the framework of the effective Hamiltonian method.

Both the Goody method ${ }^{[139]}$ and the Malkmus exponentialtailed line intensity distribution function ${ }^{[43]}$ were used in the SNB model. The spectral ranges for $\mathrm{H}_{2} \mathrm{O}, \mathrm{CO}_{2}, \mathrm{CO}$ and $\mathrm{CH}_{4}$ were $50-11250 \mathrm{~cm}^{-1}, 250-8300 \mathrm{~cm}^{-1}, 1600-6425 \mathrm{~cm}^{-1}$ and $25-$ $6200 \mathrm{~cm}^{-1}$, respectively. The transmissivities for these four gas species at different temperatures were calculated by LBL, old $\mathrm{SNB}^{[56]}$ and new SNB models. As shown in Fig. 9, the comparison between the old and new SNB results shows that the difference between them increased with rising temperatures. This behavior reflected the differences between the old and new spectral database, as well as differences in the

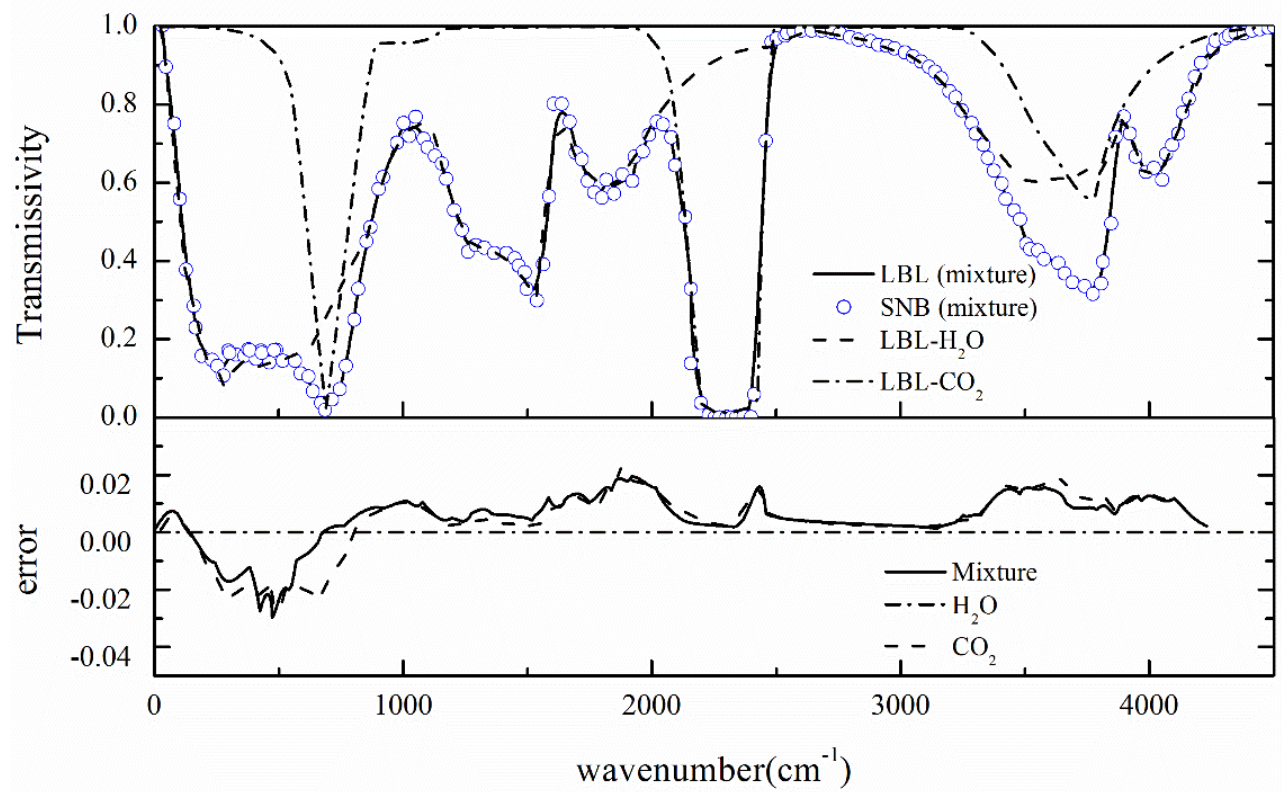

Fig. 10 Transmissivity of an isothermal and homogeneous column of $\mathrm{H}_{2} \mathrm{O}$ and $\mathrm{CO}_{2}$ mixture with $X_{\mathrm{H} 2 \mathrm{O}}=0.2, X_{\mathrm{CO} 2}=0.1, L=100 \mathrm{~cm}, \mathrm{~T}$ $=2000 \mathrm{~K}$ and $\mathrm{P}=1 \mathrm{~atm}$. (Reproduced with the permission form [57]. Copyright $@ 2012$ Elsevier Ltd.) 
internal partition functions. These differences were attributed to the lack of hot lines above $1500 \mathrm{~K}$ in the old EM2C database ${ }^{[158]}$ for $\mathrm{CO}_{2}$ at the spectral region $1900-2350 \mathrm{~cm}^{-1}$. After getting the new SNB parameters for $\mathrm{H}_{2} \mathrm{O}, \mathrm{CO}_{2}, \mathrm{CO}$ and $\mathrm{CH}_{4}$, they checked the accuracy of the SNB model in the case of variable compositions and mole fractions in the mixture. The narrow-band transmissivities calculated by the new SNB and LBL models as well as the relative errors of the two models for $\mathrm{H}_{2} \mathrm{O}$ and $\mathrm{CO}_{2}$ mixtures when $X_{\mathrm{H} 2 \mathrm{O}}=0.2$ and $X_{\mathrm{CO} 2}=0.1$ at $\mathrm{T}$ $=2000 \mathrm{~K}$ are shown in Fig. 10. The transmissivity of the mixed gas was calculated by the product of individual transmissivity based on the uncorrelation assumption, which has been proved in Ref. ${ }^{[146]}$ Fig. 10 shows the new SNB predictions agreed very well with the LBL predictions with errors below $3 \%$. The transmissivity product model does not introduce higher errors than the differences calculated for the pure species.

To investigate the radiative contribution of the gaseous fuels in the radiative feedback toward the pool fire surface, Consalvi and $\mathrm{Liu}^{[58]}$ established the Malkmus narrow band parameters for nine fuels (methane, methanol, ethane, ethylene, propane, propylene, heptane, methyl methacrylate and toluene) based on the NIST measurements. ${ }^{[163-165]}$ The total emissivities of methane, defined as $\varepsilon=\frac{1}{I_{b}(T)} \int_{0}^{\infty}(1-$ $\left.\exp \left(-\kappa_{\eta} L\right)\right) I_{b \eta}(T) d \eta$ were calculated by the NB database, the NIST database, the EM2C database, ${ }^{[162]}$ HITRAN 2012 and the data from Brosmer and Tien ${ }^{[166]}$ and compared in Fig. 11 for assessing the accuracy of the NB database. It can be seen that the NB database predictions agree very well with the other four database predictions below $600 \mathrm{~K}$, while the results calculated by the HITRAN 2012 are lower than those by the other databases above $600 \mathrm{~K}$. Subsequently, the distributions of temperature and concentration in the axis of methane pool fires ${ }^{[167,168]}$ were extracted and the radiative intensity distributions of pool fires were calculated by the proposed
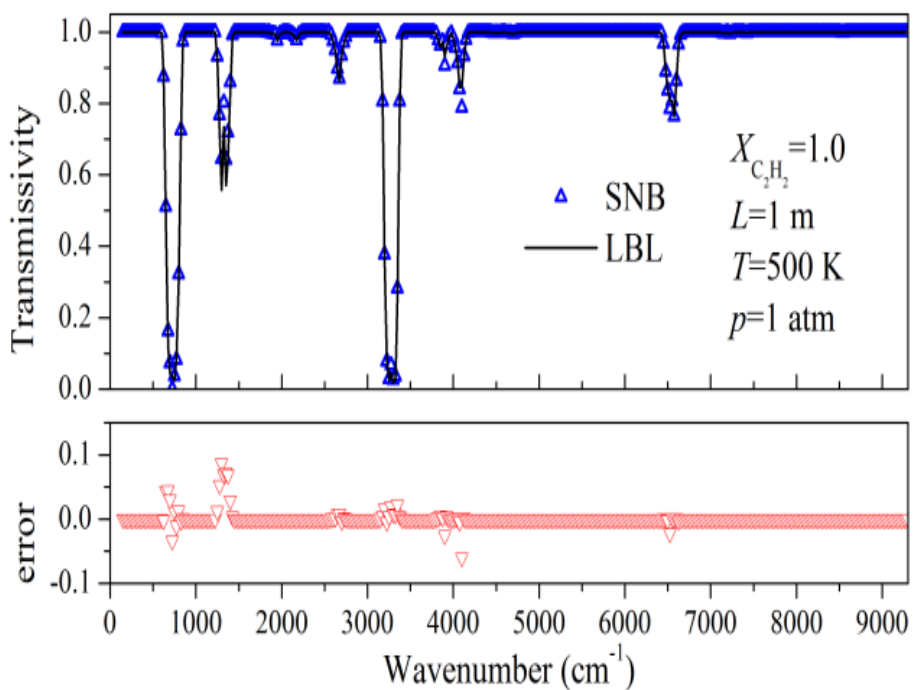

(a)
Malkmus NB database, considering the methane radiation reabsorption.

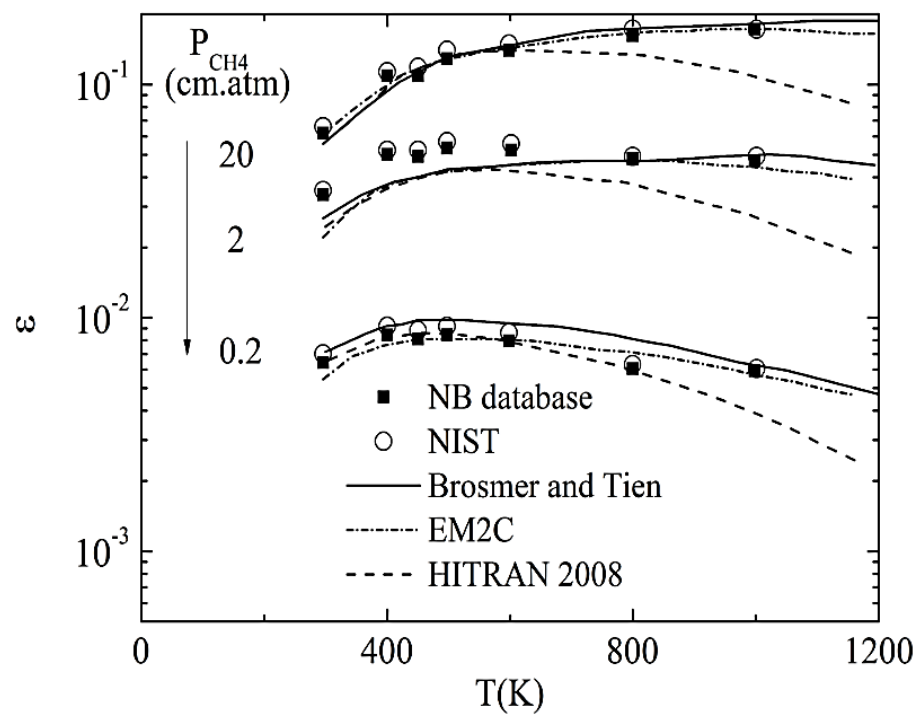

Fig. 11 The total emissivities of $\mathrm{CH}_{4}$ calculated by the NB database, the NIST database, the EM2C database, HITRAN 2012 and the data from Brosmer and Tien. ${ }^{[166]} \mathrm{CH}_{4}$ was diluted by $\mathrm{N}_{2}$ with a total pressure of $1 \mathrm{~atm}$. (Reproduced with the permission form [58]. Copyright@2012 Elsevier Ltd.)

To investigate the radiation involvement of hydrocarbon $\mathrm{C}_{2} \mathrm{H}_{4}$ and its partial oxidation product $\mathrm{C}_{2} \mathrm{H}_{2}$ on soot formation in flames, Qi et al. ${ }^{[59]}$ generated the SNB model parameters for $\mathrm{C}_{2} \mathrm{H}_{4}$ and $\mathrm{C}_{2} \mathrm{H}_{2}$ based on HITRAN 2012. The radiation characteristics of $\mathrm{C}_{2} \mathrm{H}_{2}$ covered all of the 367 narrow bands between 150 and $9300 \mathrm{~cm}^{-1}$ while $\mathrm{C}_{2} \mathrm{H}_{4}$ had 52 narrow bands in the two spectral regions: $675-1550 \mathrm{~cm}^{-1}$ (36 bands) and $2900-3275 \mathrm{~cm}^{-1}$ (16 bands). The narrow-band transmissivities of $\mathrm{C}_{2} \mathrm{H}_{2}$ and $\mathrm{C}_{2} \mathrm{H}_{4}$ calculated by the SNB and LBL models are compared in Fig. 12. The results calculated by the LBL and SNB agree well with each other, with maximum absolute error of $0.87 \%$.

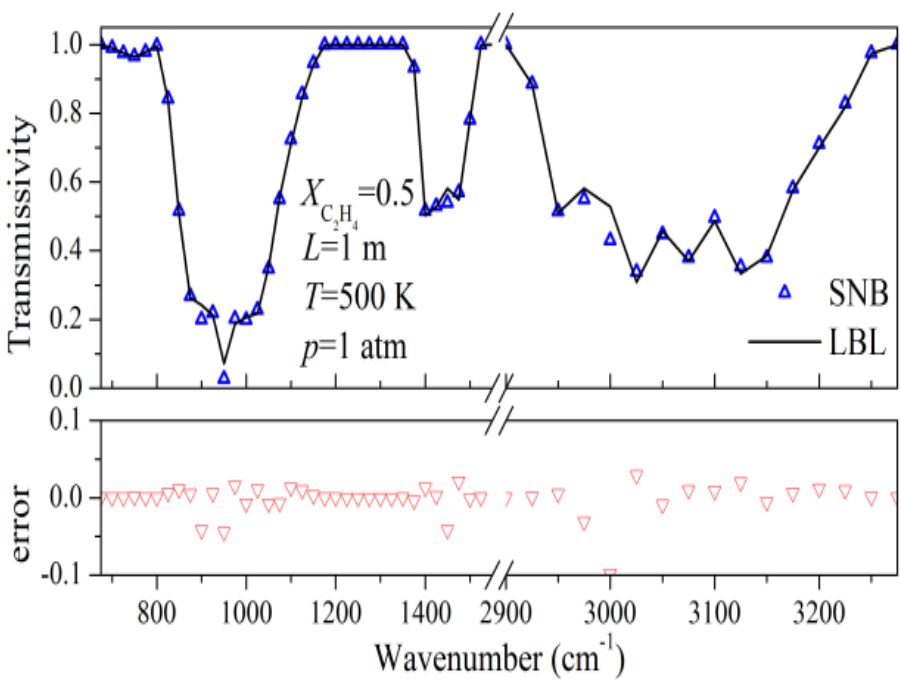

(b)

Fig. $12 \mathrm{C}_{2} \mathrm{H}_{2}$ and $\mathrm{C}_{2} \mathrm{H}_{4}$ transmissivities along an isothermal and homogeneous path: (a) $X_{\mathrm{C} 2 \mathrm{H} 2}=1.0, L=1 \mathrm{~m}, T=500 \mathrm{~K}$; (b) $X_{\mathrm{C} 2 \mathrm{H} 4}=0.5$, $L=1 \mathrm{~m}, T=500 \mathrm{~K}$. (Reproduced with the permission form [59]. Copyright@2016 Elsevier Ltd.) 


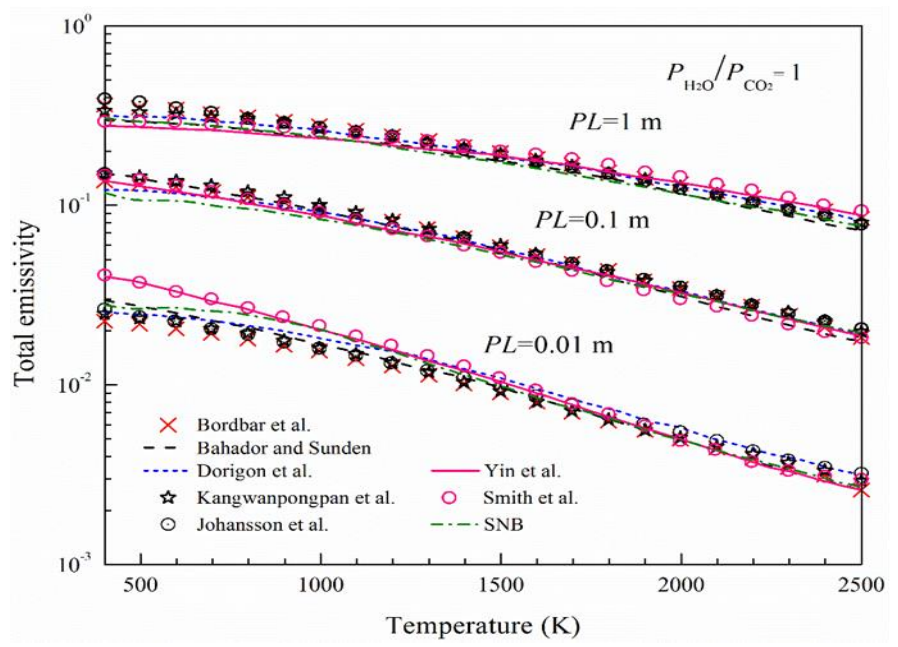

(a)

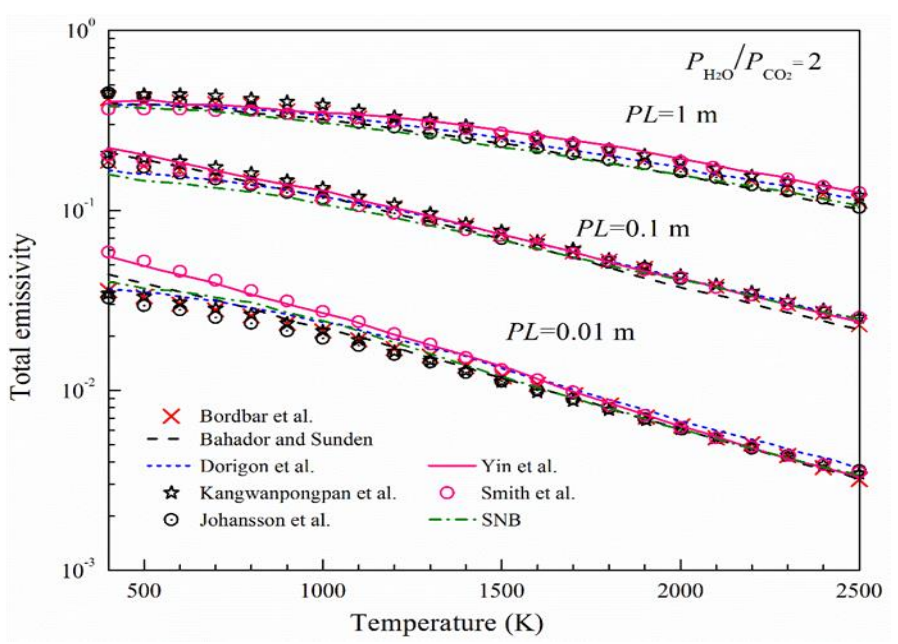

(b)

Fig. 13 The distributions of the total emissivity as a function of temperature calculated by different WSGG models at two pressure ratios (a) $P_{\mathrm{H} 2 \mathrm{O}} / P_{\mathrm{CO} 2}=1$; (b) $P_{\mathrm{H} 2 \mathrm{O}} / P_{\mathrm{CO} 2}=2 .{ }^{[147]}$

The data of temperatures and gas species concentrations along the axis of a laminar ethylene/air diffusion flame with 194 $\mathrm{mL} / \mathrm{min}_{2} \mathrm{H}_{4}$ and $284 \mathrm{~L} / \mathrm{min}$ air ${ }^{[169,170]}$ were extracted, and the mole fractions and temperatures along the centerline of the flame were used to fill a $1 \mathrm{D}$ enclosure. The radiative heat fluxes and sources were studied in this 1D enclosure. The results showed that although $\mathrm{CO}_{2}$ and $\mathrm{H}_{2} \mathrm{O}$ dominated the radiative heat transfer process, at the base of the flame, the two hydrocarbons, $\mathrm{C}_{2} \mathrm{H}_{2}$ and $\mathrm{C}_{2} \mathrm{H}_{4}$, together contributed nearly two fifth of the heat sources.

Table 4. Summary of different WSGG model coefficients. ${ }^{[147]}$

\begin{tabular}{|c|c|c|c|c|}
\hline & $\begin{array}{l}\text { Temperature } \\
\text { range }(\mathrm{K})\end{array}$ & $P_{\mathrm{H} 2 \mathrm{O}} / P_{\mathrm{CO} 2}$ & $\begin{array}{l}\text { pressure } \\
\text { path- } \\
\text { length } \\
(\mathrm{atm} \cdot \mathrm{m})\end{array}$ & $\begin{array}{l}\text { Referen } \\
\text { ce } \\
\text { model }\end{array}$ \\
\hline Smith et al. ${ }^{[70]}$ & $600-2400$ & 1,2 & $0.001-10$ & EWB \\
\hline $\begin{array}{l}\text { Bahador and } \\
\text { Sunden }^{[171]}\end{array}$ & $500-2500$ & 1,2 & $0.001-10$ & LBL \\
\hline $\begin{array}{l}\text { Johansson et } \\
a l .^{[149]}\end{array}$ & $500-2500$ & $0.125-2$ & $0.001-60$ & SNB \\
\hline Yin et al. ${ }^{[172]}$ & $500-3000$ & $\begin{array}{l}0.125, \\
0.25, \quad 0.5, \\
0.75,1,2, \\
4\end{array}$ & $0.001-60$ & LBL \\
\hline $\begin{array}{l}\text { Kangwanpong } \\
\text { pan et al. }{ }^{[173]}\end{array}$ & $400-2500$ & $0.125-4$ & $0.001-60$ & LBL \\
\hline $\begin{array}{l}\text { Dorigon et } \\
a l .^{[75]}\end{array}$ & $400-2500$ & 1,2 & $\begin{array}{l}0.0001- \\
10\end{array}$ & LBL \\
\hline $\begin{array}{l}\text { Bordbar et } \\
a l^{[148]}\end{array}$ & $300-2400$ & $0.01-4$ & $0.001-60$ & LBL \\
\hline
\end{tabular}

\subsection{WSGG model coefficients}

In the WSGG model, the total emissivities are calculated by the LBL. Subsequently, fitting Eq. (26) yields the absorption coefficient $k_{i}$ and the weighting factor $a_{i}$. So far, many researchers have proposed different parameters based on different molar fraction ratios of $\mathrm{H}_{2} \mathrm{O}$ and $\mathrm{CO}_{2}$ using different databases, as summarized in Table 4.

Fig. 13 compares the total emissivities calculated by the WSGG model coefficients of Table 4 with the SNB benchmark predictions at two pressure ratios (a) $P_{\mathrm{H} 2 \mathrm{O}} / P_{\mathrm{CO} 2}=1$; (b) $P_{\mathrm{H} 2 \mathrm{O}} / P_{\mathrm{CO} 2}=2$ for temperatures between $400 \mathrm{~K}$ and $2500 \mathrm{~K}$, and for three pressure path-lengths: $1.0,0.1$ and $0.01 \mathrm{~atm} \cdot \mathrm{m}$. It can be seen that the predictions of all the references are in excellent agreement with the SNB prediction at high pressure path-length, while the predictions of Yin et al. ${ }^{[172]}$ and Smith et $a l .{ }^{[70]}$ are quite different from the benchmark model at low pressure path-length. With the increase of $P_{\mathrm{H} 2 \mathrm{O}} / P_{\mathrm{CO} 2}$, the models of Johansson et al. ${ }^{[149]}$ and Kangwanpongpan et al. ${ }^{[173]}$ yield more favorable predictions at high temperatures.

\section{Discussion}

In this section, the numerical calculations are conducted for several cases using several gas radiation models and the RTE solution methods reviewed in the past Sections, for $1 \mathrm{D}, 2 \mathrm{D}$ and 3D systems.

\section{$5.11 \mathrm{D}$ cases}

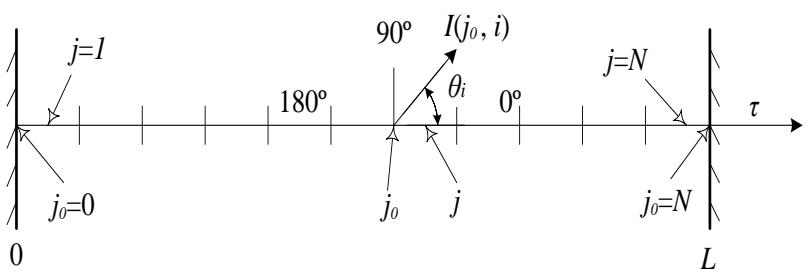

Fig. 14 A parallel-plane medium with two boundaries.

To evaluate the accuracy and efficiency of the gas radiation models and RTE solutions, the simplest case 1D plane-parallel medium was first investigated. As shown in Fig. 14, various absorbing-emitting gas mixtures $\left(\mathrm{H}_{2} \mathrm{O}, \mathrm{CO}_{2}, \mathrm{CO}\right.$, et al.) were studied when calculating the thermal radiation heat transfer in 
a one-dimensional layer. The thickness of the medium was $L$ homogeneous/inhomogeneous gases. For all the cases, the and the medium was divided uniformly into $N$ total pressure is set as 1 atm and the emissivity of the two walls elementsnumbered by $j=1 \ldots N$. The polar angle $\theta$ (in $[0, \pi]$ ) is 1.0 . The temperatures and gas species mole fractions are was divided uniformly into $M$ parts, and the angular shown in Table 5. Case 5 is an air combustion simulation, in

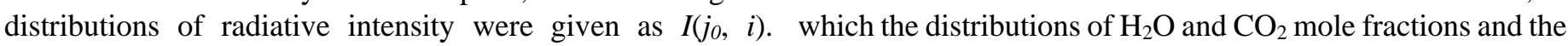
Numerical calculations were carried out at the different spatial and angular discretization for different methods.

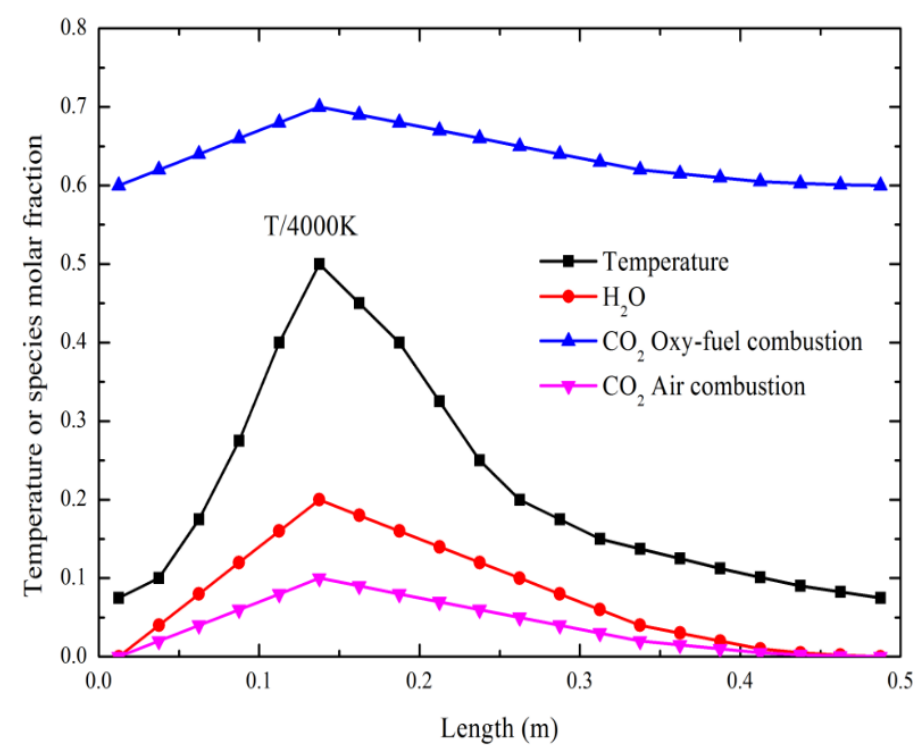

Fig. 15 The temperature and gas species mole fraction distributions of Cases 5-6 in Table 5 for air and oxy-fuel combustion. (Reproduced with the permission form [31]. Copyright@2017 Elsevier Ltd.)

Several classical cases are discussed for one-dimensional planar with isothermal/nonisothermal,

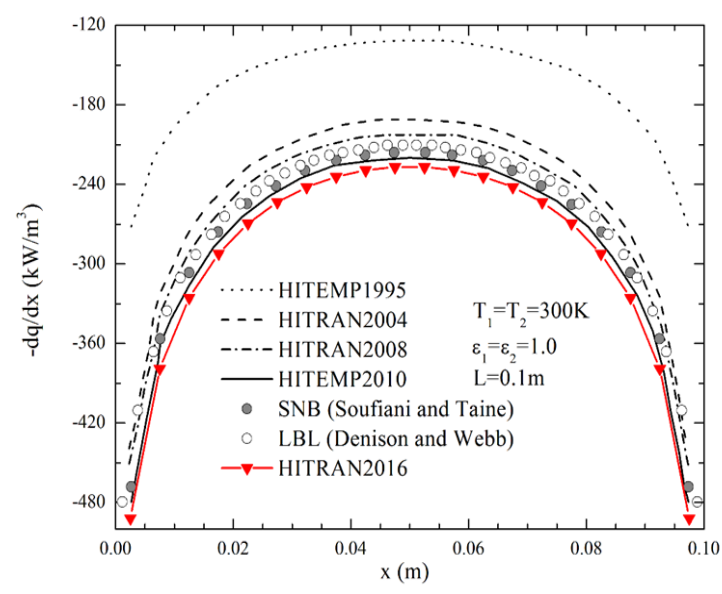

(a) temperature are given in Ref. ${ }^{[144]}$ Case 6 is an oxy-fuel combustion simulation, in which the $\mathrm{CO}_{2}$ mole fraction is larger than the $\mathrm{H}_{2} \mathrm{O}$ mole fraction while the temperature is unchanged.

Table 5. Simulated test cases. ${ }^{[31,174]}$

\begin{tabular}{llllll}
\hline Case & Medium & $L(\mathrm{~m})$ & $T(\mathrm{~K})$ & $X_{\mathrm{H} 2 \mathrm{O}}$ & $X_{\mathrm{CO} 2}$ \\
1 & $\mathrm{H}_{2} \mathrm{O}$ & 0.1 & 1000 & 1.0 & - \\
2 & $\mathrm{CO}_{2}$ & 0.1 & 2000 & - & 0.5 \\
3 & $\mathrm{CO}_{2}$ & 0.5 & 1500 & - & 0.1 \\
4 & $\mathrm{H}_{2} \mathrm{O}$ & 1.0 & 1000 & $4(1-$ & - \\
& & & & $x / L) x / L$ &
\end{tabular}

$\begin{array}{llllll}5 & \mathrm{H}_{2} \mathrm{O}+ & 0.5 & \text { Fig.15 } & \text { Fig.15 } & \text { Fig.15 } \\ & \mathrm{CO}_{2} & & & & \\ & \mathrm{H}_{2} \mathrm{O}+ & 0.5 & \text { Fig.15 } & \text { Fig.15 } & \text { Fig.15 } \\ \mathrm{CO}_{2} & & & & \\ & & & & & \end{array}$

In Case 1, the 1D parallel-plane layer was filled with $\mathrm{H}_{2} \mathrm{O}$, which was considered as an isothermal homogeneous medium. As shown in Fig. 16(a), the LBL results were given by ref. ${ }^{[76]}$ based on HITRAN 1992. The black line results were calculated by the LBL based on HITEMP 1995, HITRAN 2004, HITRAN 2008, and HITEMP 2010, respectively. The SNB results were calculated using the dataset of Soufiani and

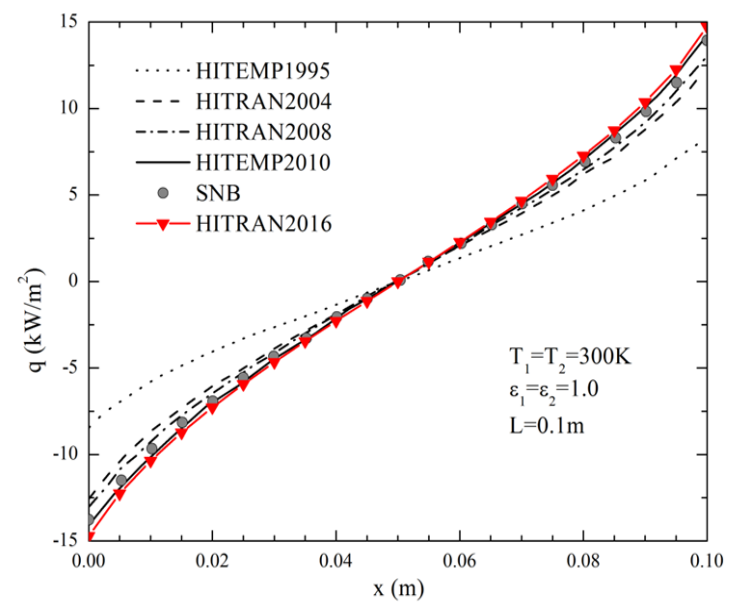

(b)

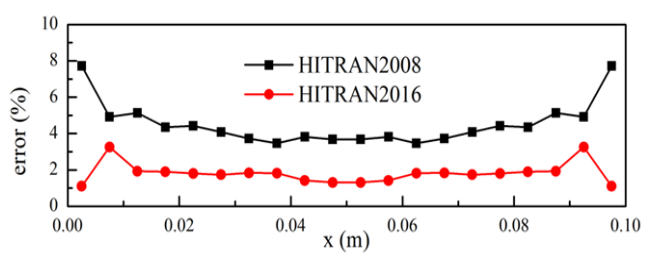

(c)

Fig. 16 Distributions of the radiative sources, heat fluxes and normalized deviations for Case 1: $T=1000 \mathrm{~K}, X_{\mathrm{H} 2 \mathrm{O}}=1.0$ and $L=0.1 \mathrm{~m}$. 

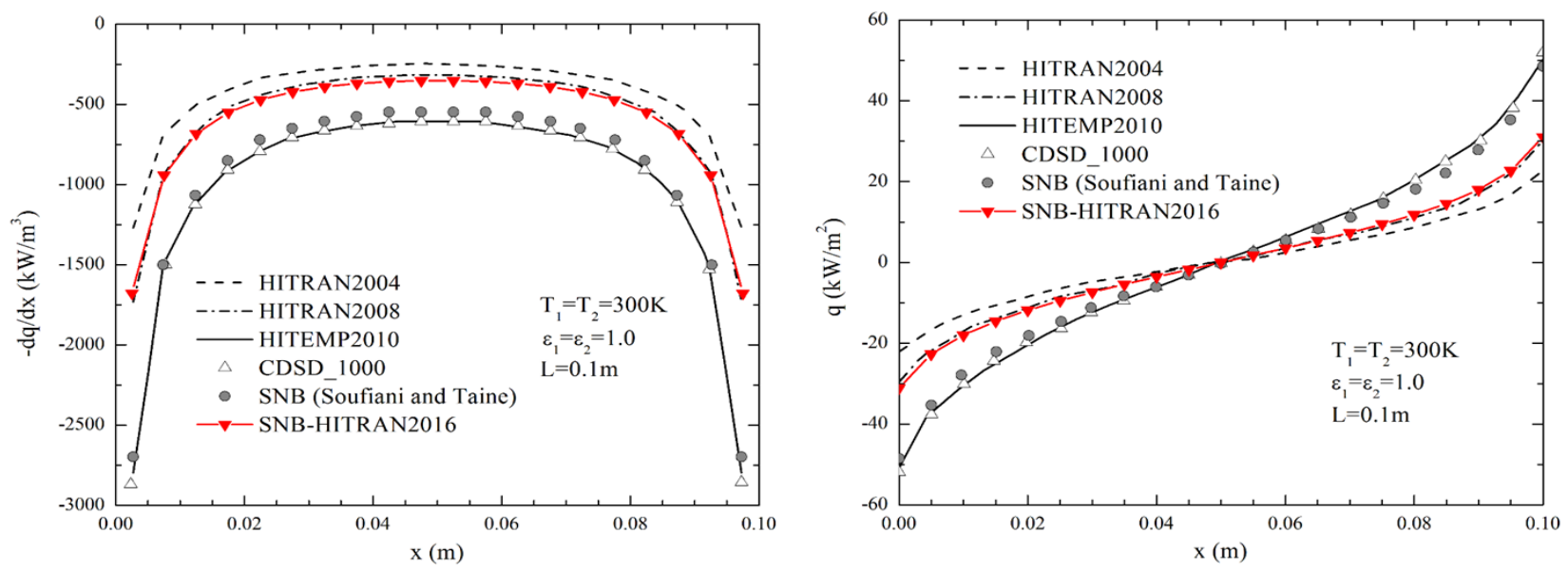

(a)

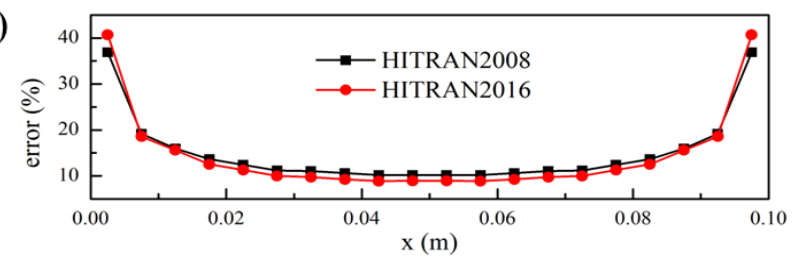

(b)

(c)

Fig. 17 Distributions of the radiative sources, heat fluxes and the normalized deviations for Case 2: $T=2000 \mathrm{~K}, X_{\mathrm{CO} 2}=0.5$ and $L=0.1$ m.

Taine. ${ }^{[56]}$ The red line results were calculated by the SNB model based on HITRAN 2016. The normalized deviation of the calculated radiative source by HITRAN 2016 and HITRAN 2008 comparing to that by HITEMP 2010, which is used as the benchmark, is defined as: $\operatorname{error}(\%)=$ $\left|\frac{\nabla \cdot q_{\text {bench }}-\nabla \cdot q_{H I T R A N}}{\nabla \cdot q_{\text {bench, } \text { max }}} \|\right|$. It can be seen that the HITRAN 2016 and SNB predictions of radiative sources were in excellent

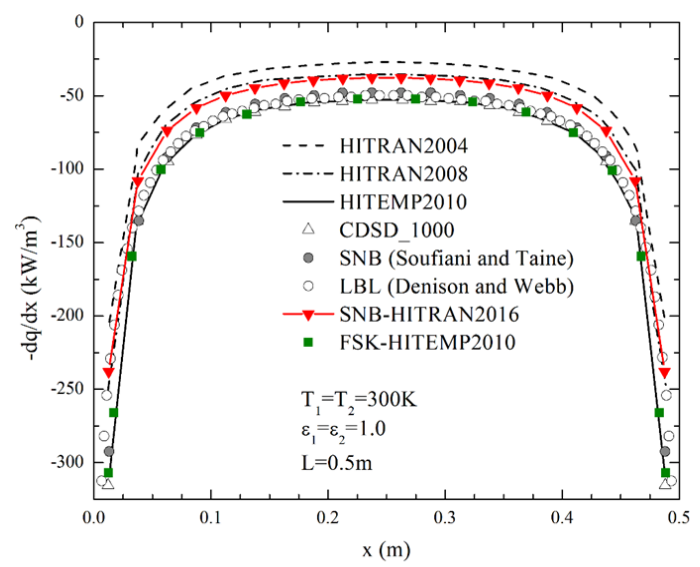

(a)
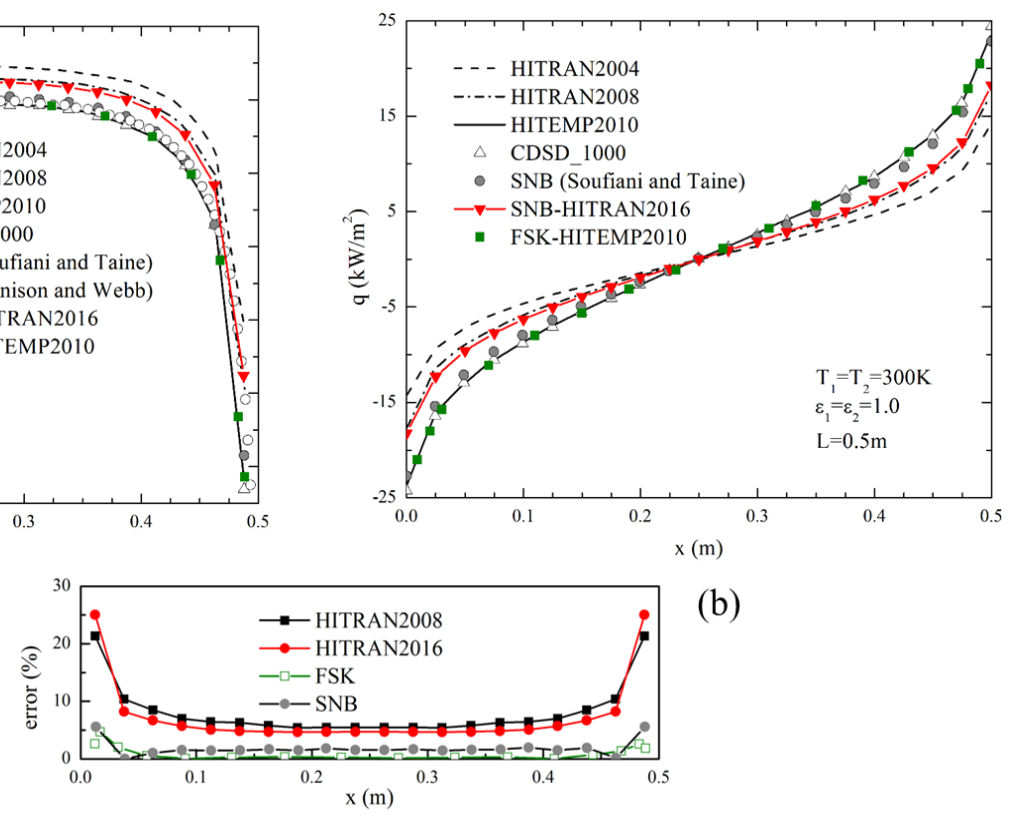

(b)

(c)

Fig. 18 Distributions of the radiative sources, heat fluxes and the normalized deviations for Case $3: T=500 \mathrm{~K}, X_{\mathrm{CO} 2}=0.1$ and $L=0.5$ m. 
In Case 2, an isothermal homogeneous medium filled with $\mathrm{CO}_{2}$ was studied and shown in Fig. 17. It can be seen that the CDSD 1000 and SNB predictions were in excellent agreement with the HITEMP 2010 predictions. However, the radiative sources calculated by the HITRAN databases were lower than those by HITEMP 2010. Fig. 17(c) shows that the average normalized deviations of HITRAN 2016 (14.54\%) was only slightly lower than those of HITRAN 2008 (15.13\%), indicating the new HITRAN database did not improve the calculations for $\mathrm{CO}_{2}$ at high temperatures.

In Case 3, the temperature and mole fraction of $\mathrm{CO}_{2}$ were reduced and the path-length was increased to $0.5 \mathrm{~m}$. The differences between the HITRAN and HITEMP predictions (see Fig. 18) are smaller than those in Fig. 17 due to the reduced grid resolution and lower temperature. The average deviations of HITRAN 2016 (7.43\%) and HITRAN 20008 $(8.21 \%)$ have significant improvements over Fig. 17(c). The FSK results given by Ref. ${ }^{[175]}$ based on HITEMP 2010 were added in Fig. 18. As show in Fig. 18(c), the average normalized deviation of FSK $(1.13 \%)$ was only lower than that of SNB (1.85\%), indicating the solution of FSK model provided closest solutions to the benchmark LBL model.

In Case 4, an isothermal inhomogeneous medium filled with $\mathrm{H}_{2} \mathrm{O}$ was studied. The mole fractions of the of $\mathrm{H}_{2} \mathrm{O}$ were set as parabolic distributions. The distributions of the radiative sources and heat fluxes calculated by LBL and SNB based on different databases are shown in Fig. 19. The results calculated

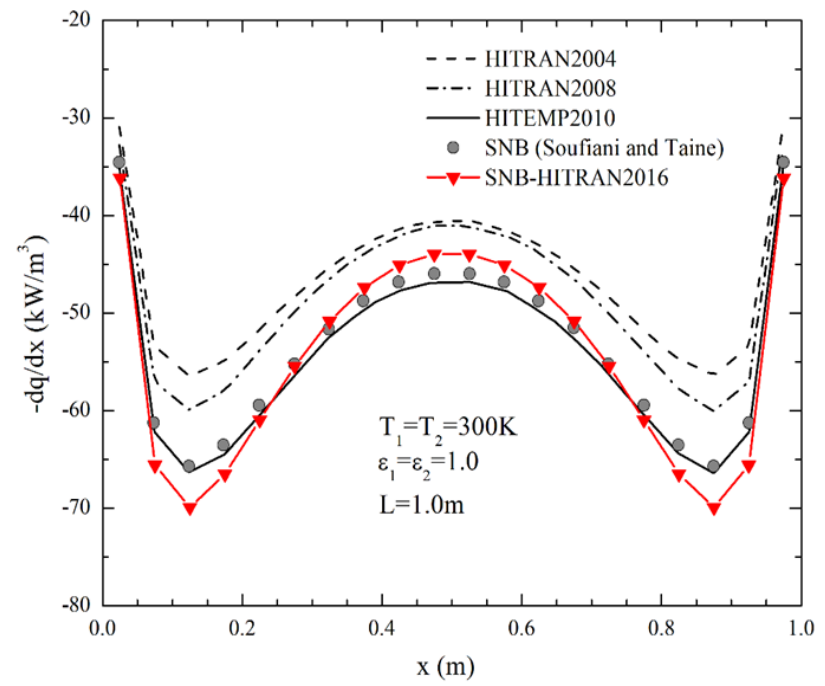

(a) by HITRAN 2016 showed better performance than those of HITRAN 2004 and HITRAN 2008. The average normalized deviation of HITRAN 2016 (2.98\%) was lower than that of HITRAN 2008 (8.65\%).

In Case 5, a non-isothermal inhomogeneous medium filled with $\mathrm{H}_{2} \mathrm{O}$ and $\mathrm{CO}_{2}$ was investigated. Distributions of temperature and gas mole fraction in Case 5 represented a counterflow fuel-air diffusion flame as given in Ref. ${ }^{[144]}$ The maximum temperature was $2000 \mathrm{~K}$ and located $x=0.14 \mathrm{~m}$. In Fig. 20(a), the radiative sources calculated by the HITRAN databases agreed well with the HITEMP results at low temperature regions, where $x=0-0.1 \mathrm{~m}$ and $0.2-0.5 \mathrm{~m}$, however, quite different in the high temperature regions. The reason is the missing hot lines of $\mathrm{CO}_{2}$ in HITRAN databases at high temperature. The SNB parameters of Soufiani and Taine ${ }^{[56]}$ contained the data from EM2C database, and hence resulted in excellent agreement with the LBL-HITEMP 2010.

Along with the development of oxy-fuel combustion technology, the $\mathrm{CO}_{2}$ concentration was increased by 6 times in Case 6, comparing to the air combustion counterpart. Due to the high $\mathrm{CO}_{2}$ concentration, the CDSD-1000 database was used in the LBL, while the spectral line parameters of $\mathrm{H}_{2} \mathrm{O}$ were taken from HITEMP 2010. The two databases were combined and denoted as HITEMP 2010+CDSD 1000 database. SNB calculations were conducted using the parameters of Soufiani and Taine ${ }^{[56]}$ and the HITRAN 2016 database. WSGG calculations were obtained from Yin et al $^{[172]}$

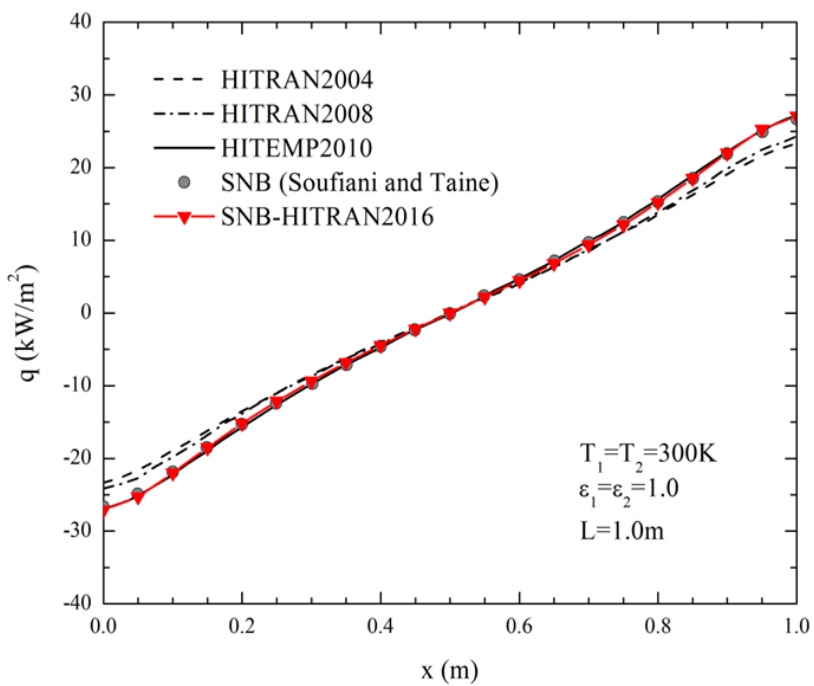

(b)

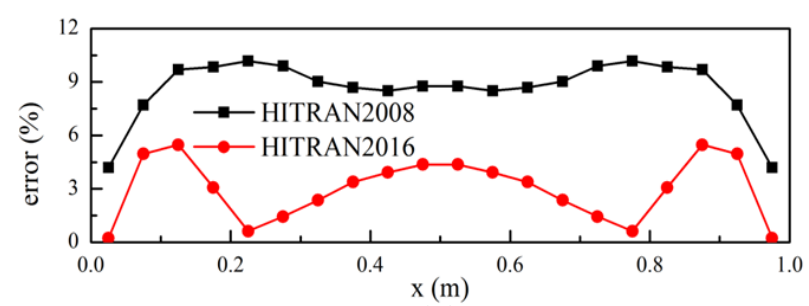

(c)

Fig. 19 Distributions of the radiative sources, heat fluxes and the normalized deviations for Case $4: T=1000 \mathrm{~K}, X_{\mathrm{H} 20}=4(1-\mathrm{x} / \mathrm{L}) \mathrm{x} / \mathrm{L}$ and $L=1.0 \mathrm{~m}$. 


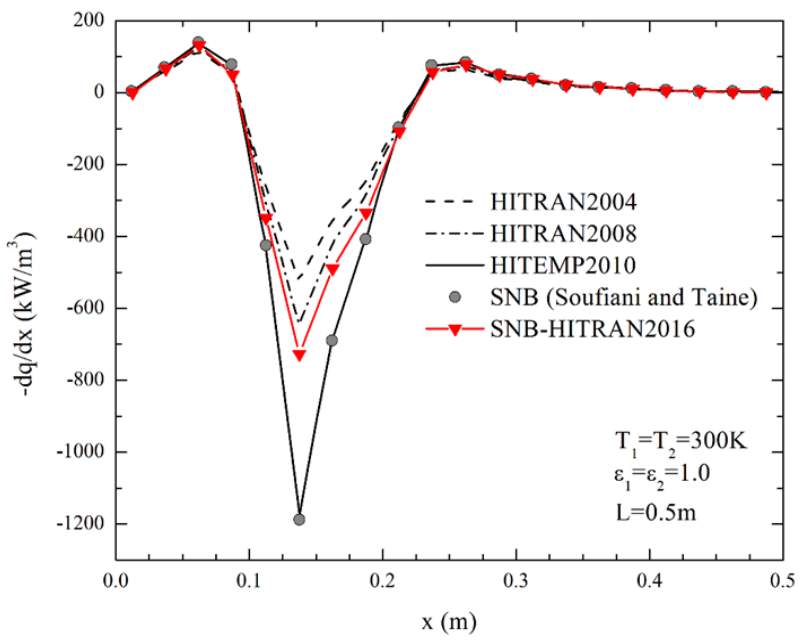

(a)

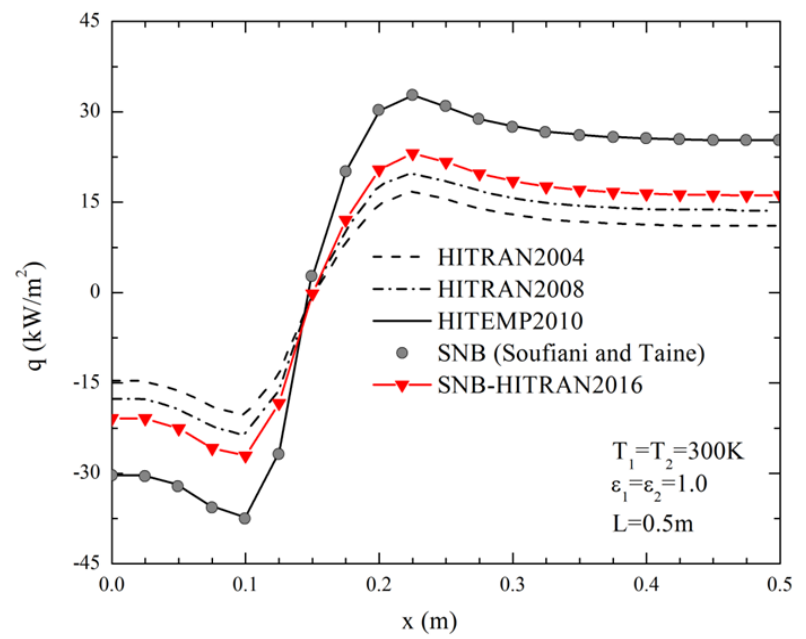

(b)

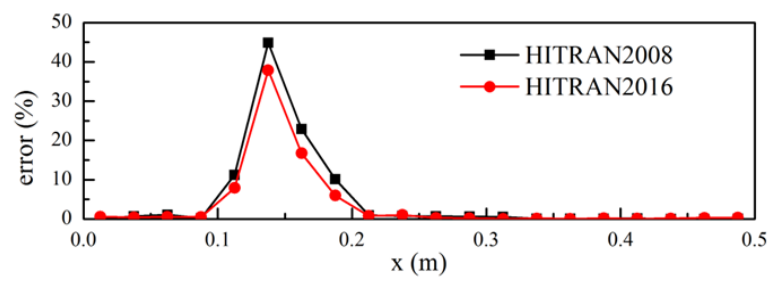

(c)

Fig. 20 Distributions of the radiative sources, heat fluxes and the normalized deviations for Case 5 (air combustion).

and Bordbar et al. ${ }^{[148]}$ The FSK results were given by Ma et excellent average normalized deviation (0.26\%), significantly $a l .{ }^{[175]}$ combining the spectral collocation method. Fig. 21(a) lower than those of other models. The average normalized shows that the LBL results calculated by HITEMP 2010 and deviation of FSK (5.31\%) was lower than those of WSGG SNB results calculated by Soufiani and Taine ${ }^{[56]}$ agreed very Bordbar et al. ${ }^{[148]}(6.07 \%)$ and WSGG Yin et al. ${ }^{[172]}(7.98 \%)$. well with the HITEMP 2010+CDSD 1000 results. As shown This indicated that FSK had better accuracy comparing to in Fig. 21(c), the SNB predictions of Soufiani and Taine ${ }^{[56]}$ had WSGG in non-isothermal inhomogeneous conditions.

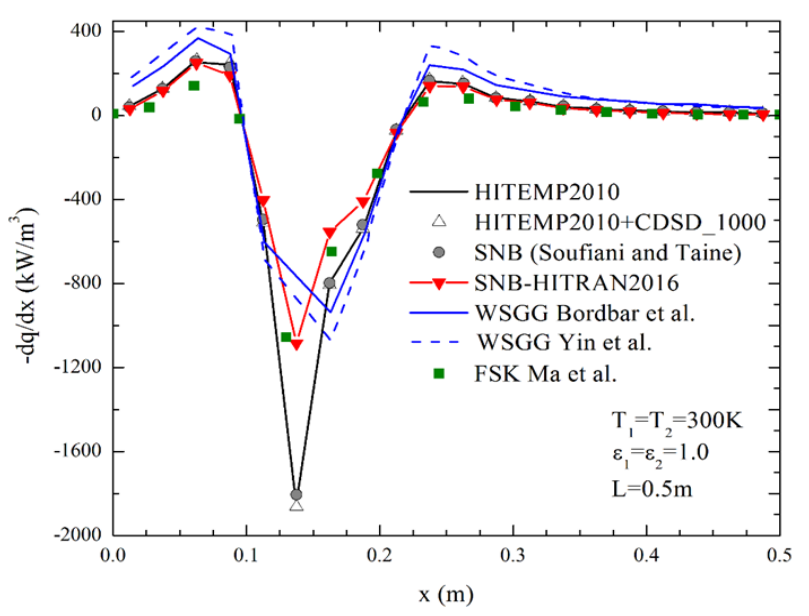

(a)

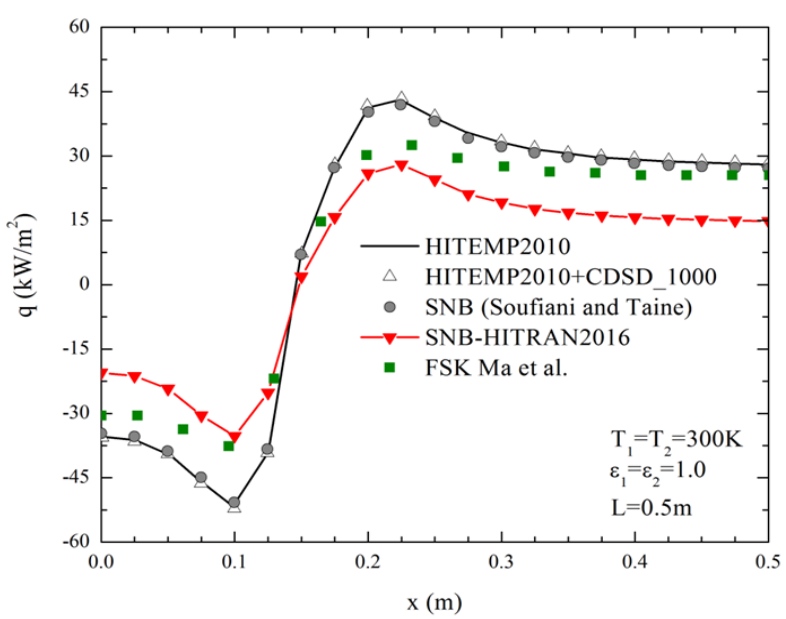

(b)

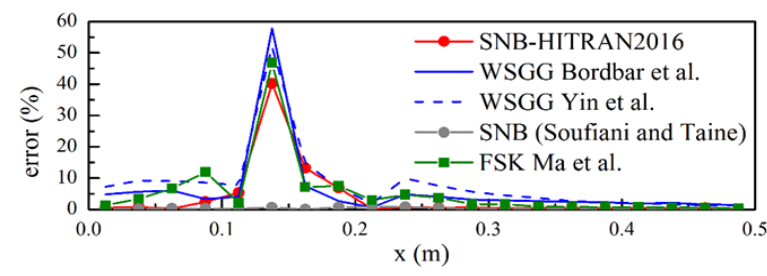

(c)

Fig. 21 Distributions of the radiative sources, heat fluxes and the normalized deviations for Case 6 (oxy-fuel combustion). 
Table 6. Temperature, mole fractions of $\mathrm{H}_{2} \mathrm{O}$ and $\mathrm{CO}_{2}$ and soot volume fractions. ${ }^{[138,176]}$

\begin{tabular}{ccccc}
\hline Case & $T(\mathrm{~K})$ & $X_{\mathrm{H} 2 \mathrm{O}}$ & $X_{\mathrm{CO} 2}$ & $X_{\text {SOOT }}$ \\
\hline 7 & {$[4 x(L-x)+0.8] \times 1000$} & $2 \times[0.4 x(L-x)+0.06]$ & $0.4 x(L-x)+0.06$ & {$[40 x(L-x)+6] \times 10^{-7}$} \\
8 & {$[4 x(L-x)+0.8] \times 1000$} & $2 \times[0.4 x(L-x)+0.06]$ & $0.4 x(x-L)+0.06$ & {$[40 x(L-x)+6] \times 10^{-8}$} \\
9 & {$[4 x(x-L)+1.8] \times 1000$} & $2 \times[0.4 x(L-x)+0.16]$ & $0.4 x(x-L)+0.16$ & {$[4 x(x-L)+1.6] \times 10^{-6}$} \\
10 & {$[4 x(x-L)+1.8] \times 1000$} & $2 \times[0.4 x(L-x)+0.16]$ & $0.4 x(x-L)+0.16$ & {$[4 x(x-L)+1.6] \times 10^{-7}$} \\
\hline
\end{tabular}

In real combustion systems, the combustion products contain not only $\mathrm{H}_{2} \mathrm{O}, \mathrm{CO}_{2}$ and $\mathrm{CO}$ but also soot. Moreover, the boundaries cannot be ideal black cold walls. Due to the band radiation of the gases combined with the continuous radiation of the soot and the existence of wall reflection, it is hard to calculate the radiative heat transfer accurately for gas and soot mixtures. Hence, four cases with $\mathrm{H}_{2} \mathrm{O}, \mathrm{CO}_{2}$ and soot mixtures are reviewed.

The cases were summarized in Table 6: the mole fraction of $\mathrm{H}_{2} \mathrm{O}$ was twice the mole fraction of $\mathrm{CO}_{2}$ and the soot volume fractions were taken from Bressloff. ${ }^{[176]}$ The SNB parameters of $\mathrm{H}_{2} \mathrm{O}$ and $\mathrm{CO}_{2}$ were provided by Soufiani and Taine ${ }^{[56]}$ and the soot absorption coefficient was developed by Hottel and Sarofim. ${ }^{[67]}$ The radiative sources calculated by the

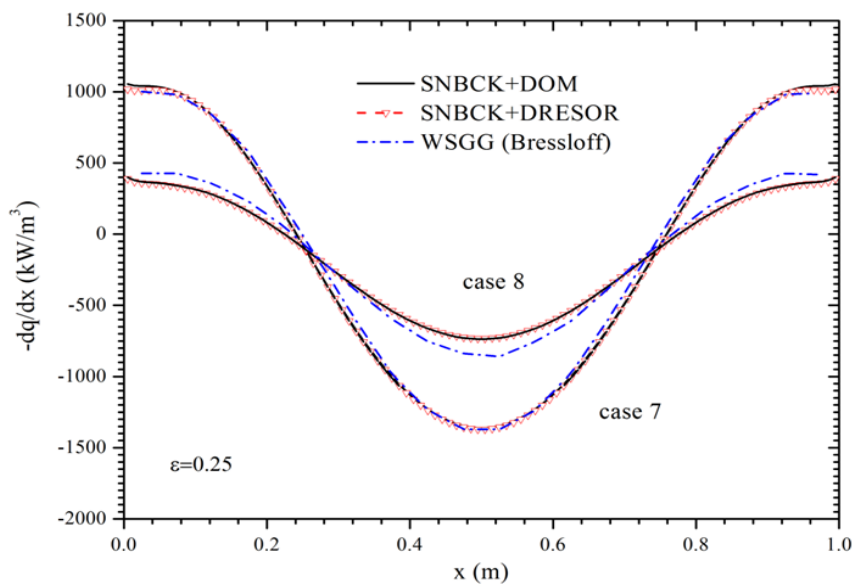

(a)
SNBCK/DOM, SNBCK/DRESOR ${ }^{[138]}$ and $\mathrm{WSGG}^{[176]}$ for a non-isothermal inhomogeneous medium with cold walls $\varepsilon=0.25$ are shown in Fig. 22(a). Compared to the low soot volume fraction Case 8 , both the radiation absorption at the boundary and the radiation emission of the mixture at the middle length were enhanced in the high soot volume fraction Case 7. As shown in Fig. 22(b), the SNBCK/DRESOR predictions of radiative source were in excellent agreement with the SNBCK/DOM predictions, while the radiative source calculated by the WSGG were quite different from the SNBCK/DOM predictions. The main reason for the big difference between WSGG and SNBCK was that the spectral database used in WSGG ${ }^{[176]}$ was from an outdated Ref. ${ }^{[177]}$
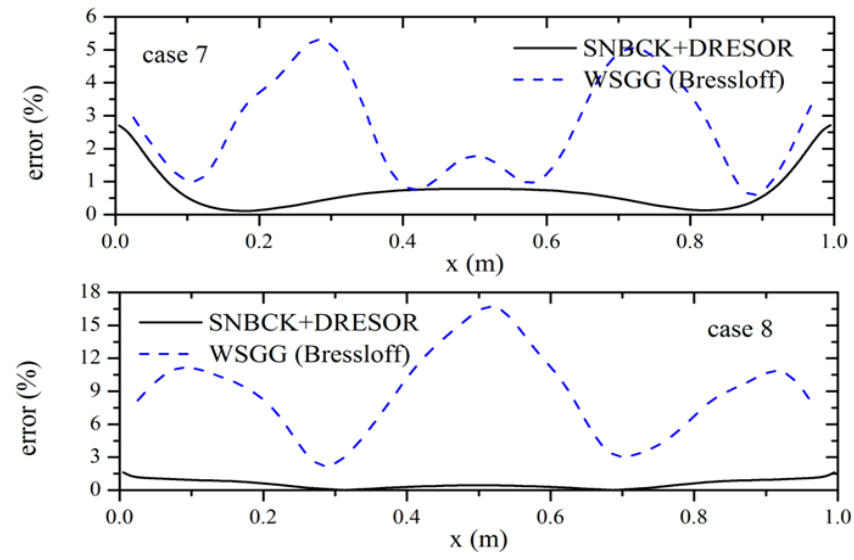

(b)

Fig. 22 Distributions of the radiative sources and the normalized deviations for Cases 7 and 8 with wall temperature $0 \mathrm{~K}$ and $\varepsilon=0.25$.

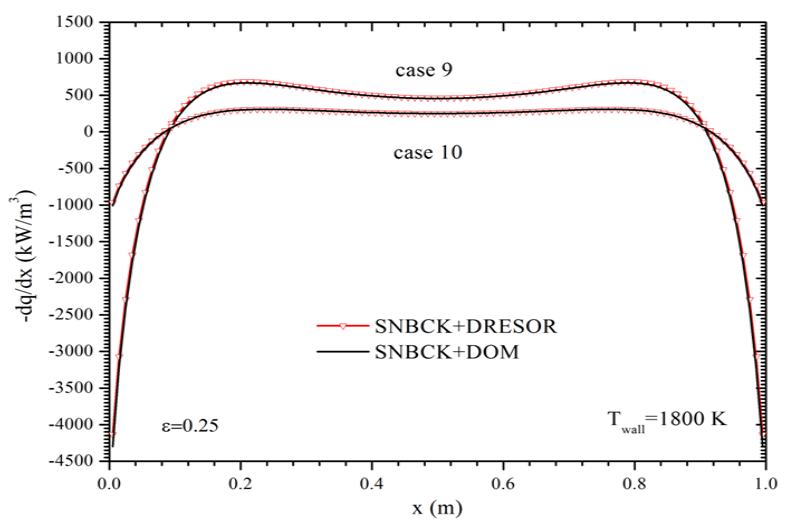

(a)

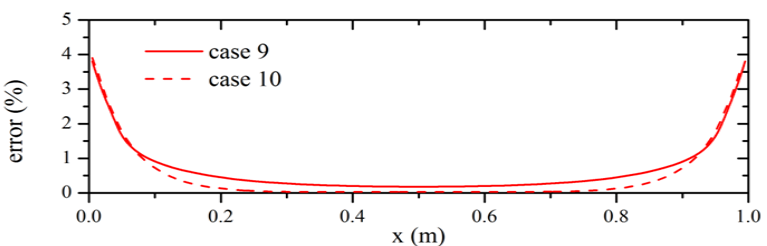

(b)

Fig. 23 Distributions of the radiative sources and the normalized deviations for Cases 9 and 10 with wall temperature $1800 \mathrm{~K}$ and $\varepsilon=0.25$. 
In Cases 9 and 10, the wall temperature was increased to $1800 \mathrm{~K}$ and the emissivity was set 0.25 . As shown in Fig. 23(a), there is a narrow area near the boundary where the emission is stronger than the absorption, for the reason that gas and soot mixture became a total absorber when the wall temperature was higher than the medium temperature. The maximum deviation of SNBCK/DRESOR was only $3.9 \%$ at the boundary. The computation time for Case 9 in Fig. 23 was $45.36 \mathrm{~s}$ using SNBCK/DRESOR after removing the computation time of the DRESOR values, while the computation time for SNBCK/DOM was $519.71 \mathrm{~s}$ using the same number of discrete directions. Consequently, SNBCK/DRESOR showed clearly advantageous computational efficiency than SNBCK/DOM in nonisothermal inhomogeneous cases.

The directional intensities at the middle position and left wall calculated by DRESOR and DOM are plotted in Fig. 24 . To keep the same computation time, we lowered the number of directions in DOM. The DRESOR predictions exhibited good agreement with the DOM predictions based on the same spectral database. More importantly, DRESOR had a higher directional resolution than DOM when using the same computation time as shown in Fig. 24.

1D spherical symmetry of the flame structure (shown in Fig. 25) is a canonical flame used to study laminar flame propagations, ${ }^{[178,179]}$ droplet combustion ${ }^{[180,181]}$ and flame speed measurements. ${ }^{[182]}$ Following discussion will be displayed on the performance of the non-gray radiation heat transfer models in this $1 \mathrm{D}$ spherical geometry for several cases. The spherical enclosure is filled with $\mathrm{H}_{2} \mathrm{O}$ and $\mathrm{CO}_{2}$. For all the spherical cases, the total pressure is $1 \mathrm{~atm}$ and the emissivity of the wall is 1.0. The spherical diameter is $1 \mathrm{~m}$, and the temperature and gas species mole fractions are given in Table 7 .

In Case 11, three gray conditions $\kappa=0.2,2,20$ were used to assess the calculation results of different RTE solution methods in the 1D spherical coordinate system. Fig. 26 shows that the P1 predictions agreed very well with the DOM predictions in the case of optically thin $\kappa=0.2$, while the relative error of P1 and DOM methods increased with the rising absorption coefficient increasing (to 2.0 and 20). Therefore, it is evident that the feature of the P1 method has an impact on its accuracy in the gray gas radiation, especially for optically thick cases.

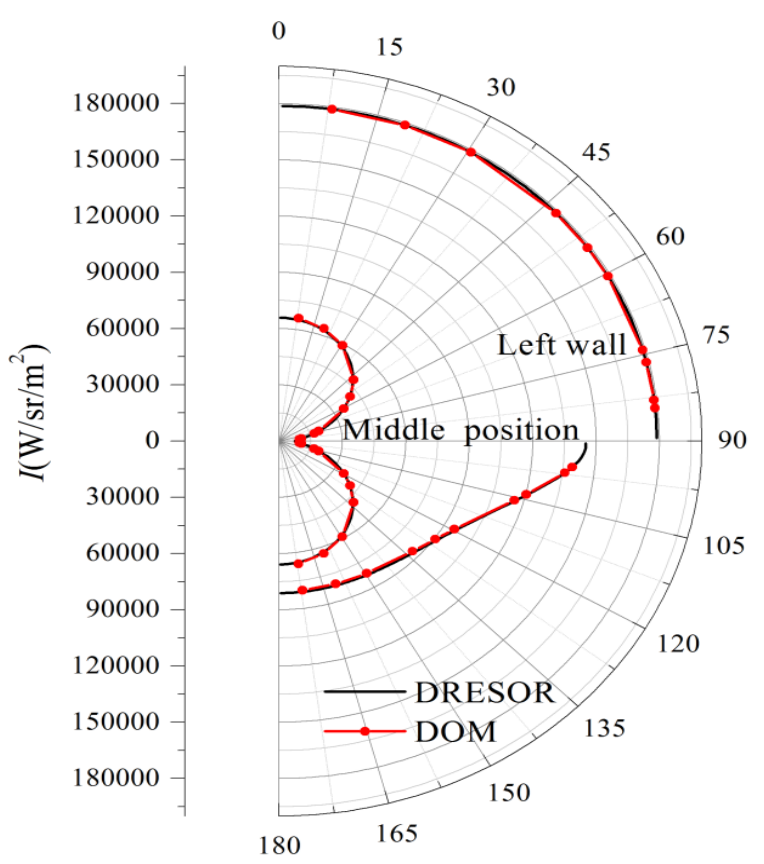

Fig. 24 Distribution of the directional intensity at the different positions calculated by DRESOR and DOM for Case 9 .

Table 7. The temperatures, mole fractions of $\mathrm{H}_{2} \mathrm{O}$ and $\mathrm{CO}_{2}$ used in 1D spherical geometry.

\begin{tabular}{lllll}
\hline Case & $T(\mathrm{~K})$ & $T_{w}(\mathrm{~K})$ & $X_{\mathrm{CO} 2}$ & $X_{\mathrm{H} 2 O}$ \\
\hline 11 & 1500 & 300 & gray & gray \\
12 & 1500 & 300 & 0.2 & - \\
13 & - & 300 & - & - \\
& $24000 r^{2}+1200$ & & $1.6 r^{2}+0.8 r+0$. & $1.6 r^{2}+0.8 r+$ \\
& $0 r+500$ & & 6 & 0.1 \\
\hline
\end{tabular}
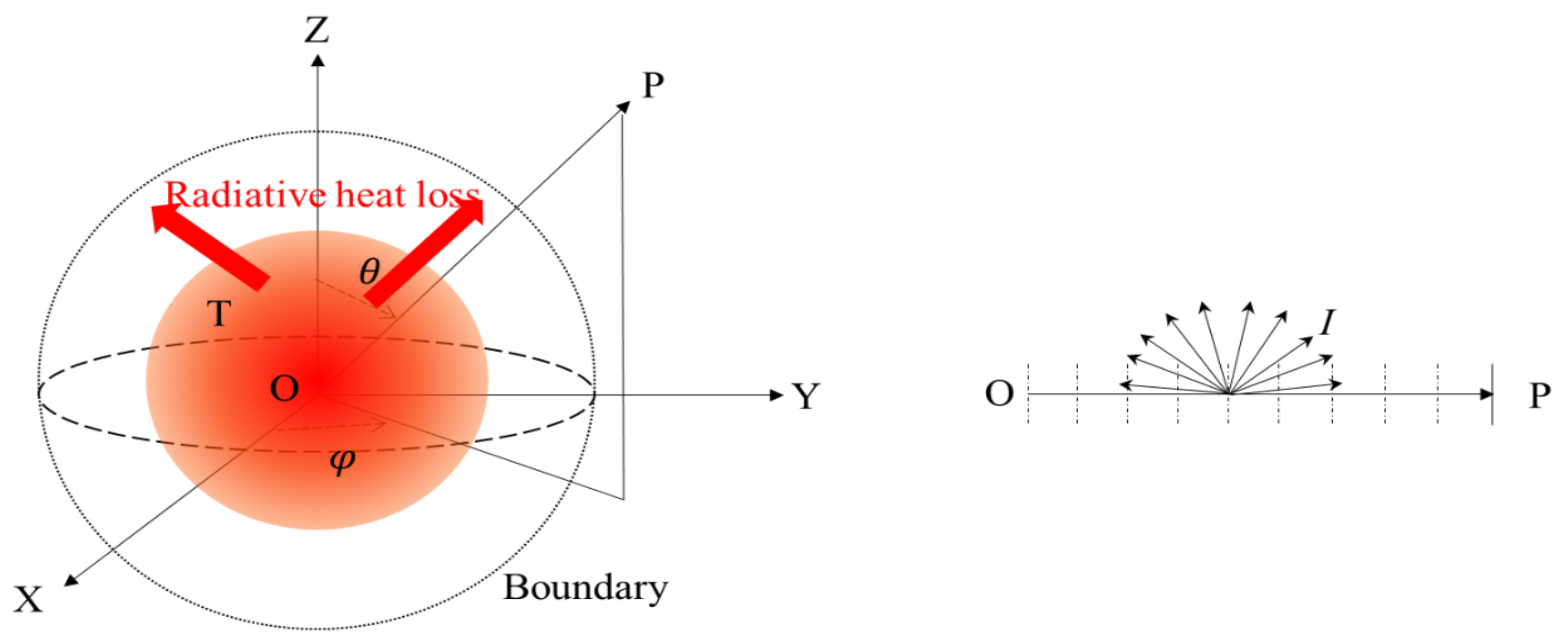

Fig. 25 Configuration for a spherical flame and 1D radiation model. (Reproduced with the permission form [183]. Copyright@2019 Elsevier Ltd.) 


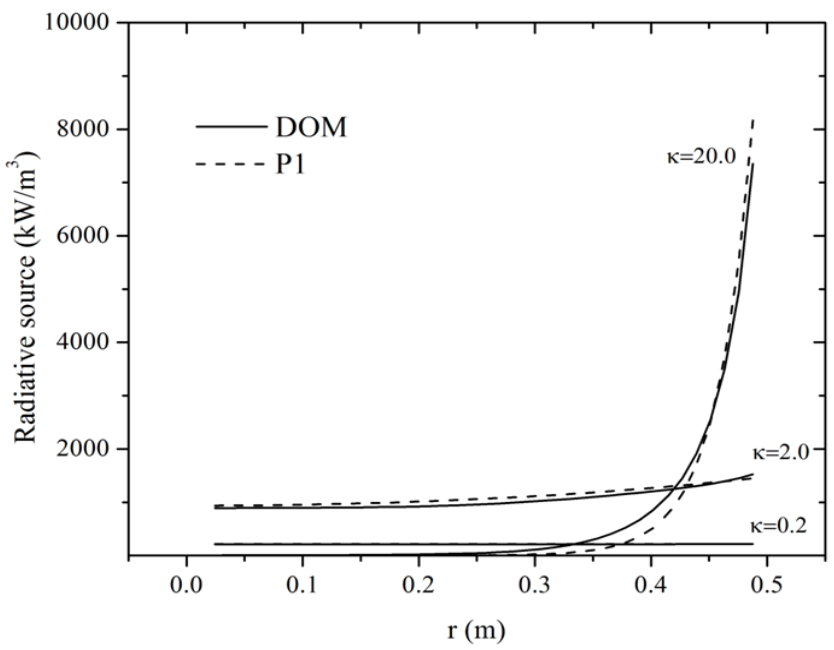

(a)

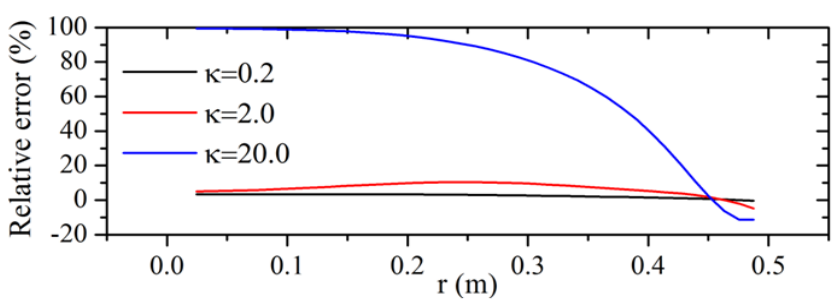

(b)

Fig. 26 Radiative heat sources and relative errors of the P1 and DOM methods for three gray cases $\kappa=0.2,2,20$ in the $1 \mathrm{D}$ spherical coordinate system.

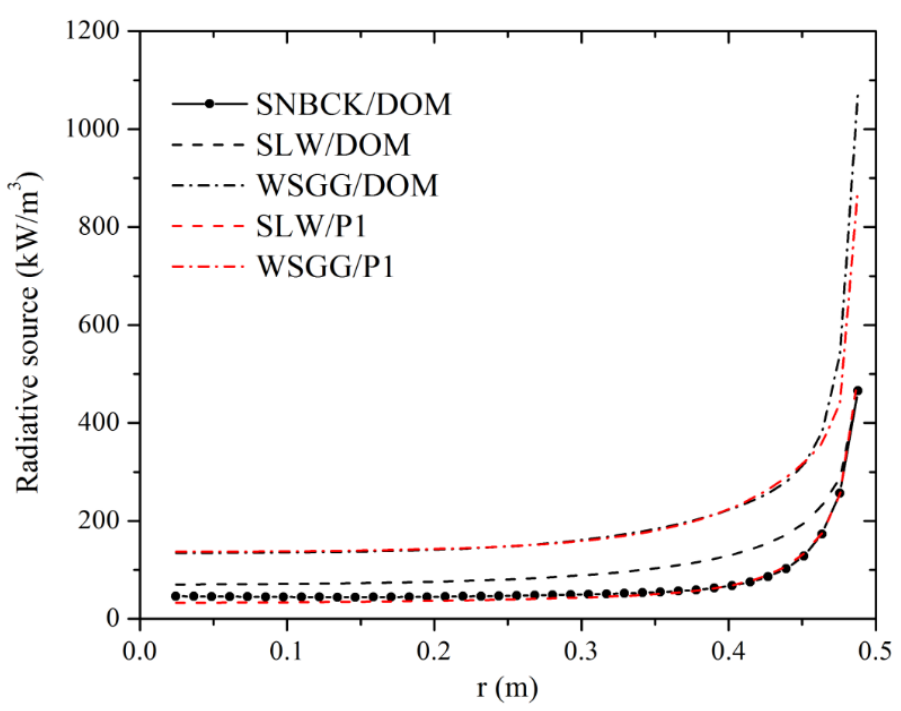

Fig. 27 Distributions of the radiative sources for Case 12: $T=1500 \mathrm{~K}, T_{w}=300 \mathrm{~K}$ and $X_{\mathrm{CO} 2}=0.2$.

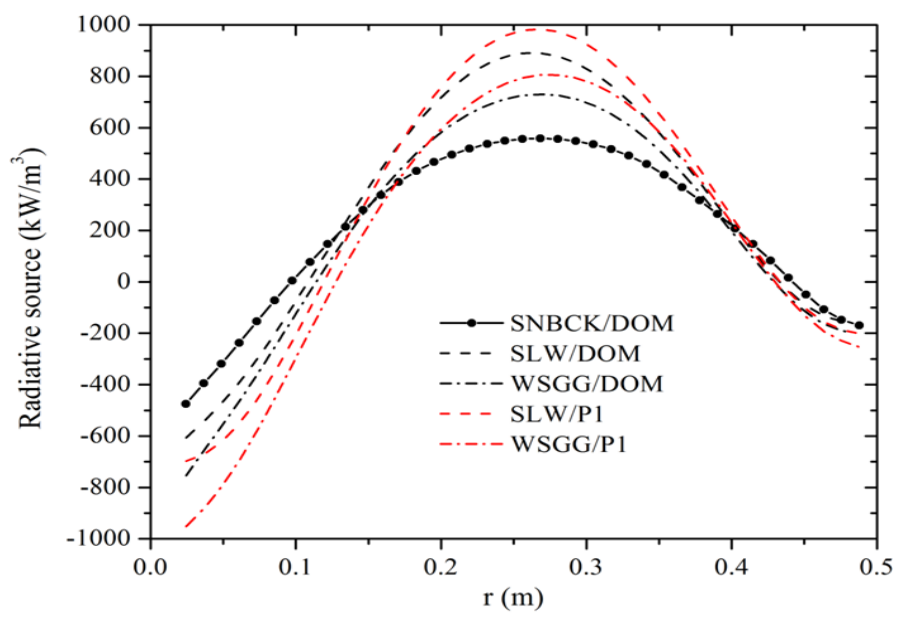

(a)
In Case 12, an isothermal homogeneous medium filled with pure $\mathrm{CO}_{2}$ was investigated with its mole concentration 0.2. The radiative sources calculated by the SNBCK/DOM, SLW/DOM, WSGG/DOM, SLW/P1 and WSGG/P1 methods are shown in Fig. 27. The results of SNBCK/DOM were used as benchmark. The SLW model used in this case was developed by Pearson and Webb ${ }^{[82]}$ based on HITEMP 2010, while the WSGG model used in this case was developed by Johansson et al. ${ }^{[149]}$ As shown in Fig. 27, the predicted radiative sources of the WSGG/DOM and WSGG/P1 were about three times that of the SNBCK/DOM. The reason is that the WSGG model is optimized for oxy-fuel $\mathrm{H}_{2} \mathrm{O}$ and $\mathrm{CO}_{2}$ conditions. The SLW/P1 predictions agreed very well with the SNBCK/DOM predictions, while the SLW/DOM overpredicted the radiative sources.

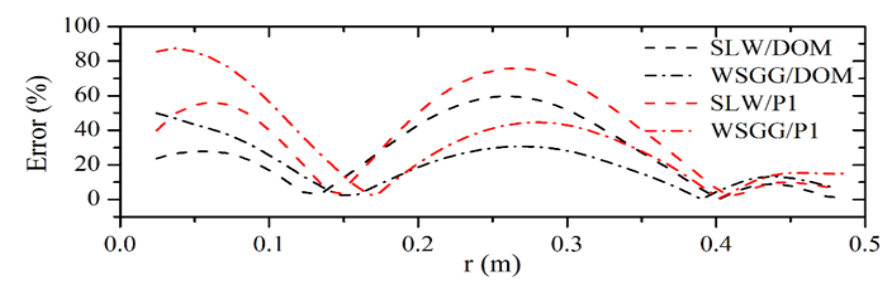

(b)

Fig. 28 Distributions of the radiative sources for Case 13: $T=-24000 r^{2}+12000 r+500, T_{w}=300 \mathrm{~K}, X_{H 2 O}=-1.6 r^{2}+0.8 r+0.1$ and $X_{C O 2}=-$ 1.6ri2 $+0.8 r+0.6$. (Reproduced with the permission form [183]. Copyright@2019 Elsevier Ltd.) 
In Case 13, a non-isotherm and inhomogeneous medium filled with $\mathrm{H}_{2} \mathrm{O}$ and $\mathrm{CO}_{2}$ was studied. The temperatures and the mole fractions of the $\mathrm{H}_{2} \mathrm{O}$ and $\mathrm{CO}_{2}$ were set as parabolic distributions. For this case, the multiplication method coupled with the reference temperature and mole fractions was needed when using the SLW model. Fig. 28 shows the predictions of SLW/DOM, WSGG/DOM, SLW/P1 and WSGG/P1 are larger than the predictions of SNBCK/DOM in the high temperature region, while an opposite trend in the low temperature region. The average errors of SLW/DOM and WSGG/DOM predictions were $26.4 \%$ and $20.03 \%$, respectively, which were less than those of SLW/P1 (37.83\%) and WSGG/P1 (34\%). Therefore, P1 performs better than DOM in the pure gas case, and on the contrary, DOM is more accurate than P1 for nonisotherm and inhomogeneous gas mixture cases.

\section{$5.22 \mathrm{D}$ cases}

The studied 2D rectangular enclosure had a dimension of 1.0 $\mathrm{m}$ length $\times 0.5 \mathrm{~m}$ width for all the cases and a boundary condition of cold black wall. Five test cases are discussed in this section and the temperature distributions and gas species mole fractions are shown in Table 8. For the non-isotherm and inhomogeneous Cases 15 and 17, the temperature and gas mole fractions were described as

$$
T(x, y)=1200 \times[0.3333(1-2|x-0.5|)(1-4 \mid y-
$$

$c(x, y)=c_{0}[4(1-2|x-0.5|)(1-4|y-0.25|)+1]$

where $c_{0}$ was 0.02 for Case 15 and 0.04 for Case 17. Fig. 29 (a) shows the temperature distributions obtained via Eq. (91), with the trend of lower edges and a higher center.

For the combustion flame Case 18, the temperature was given as follows

$T(x, y)=T_{0}+(14000 x-400)\left(1-3 y_{0}^{2}+2 y_{0}^{3}\right) x \leq 0.1$

$T(x, y)=T_{0}-\frac{10000}{9}(x-1)\left(1-3 y_{0}^{2}+2 y_{0}^{3}\right) x \geq 0.1$

where $y_{0}=|0.25-y| / 0.25$ and $T_{0}=800 \mathrm{~K}$. Fig. 29(b) displays the temperature distributions obtained via Eq. (93).

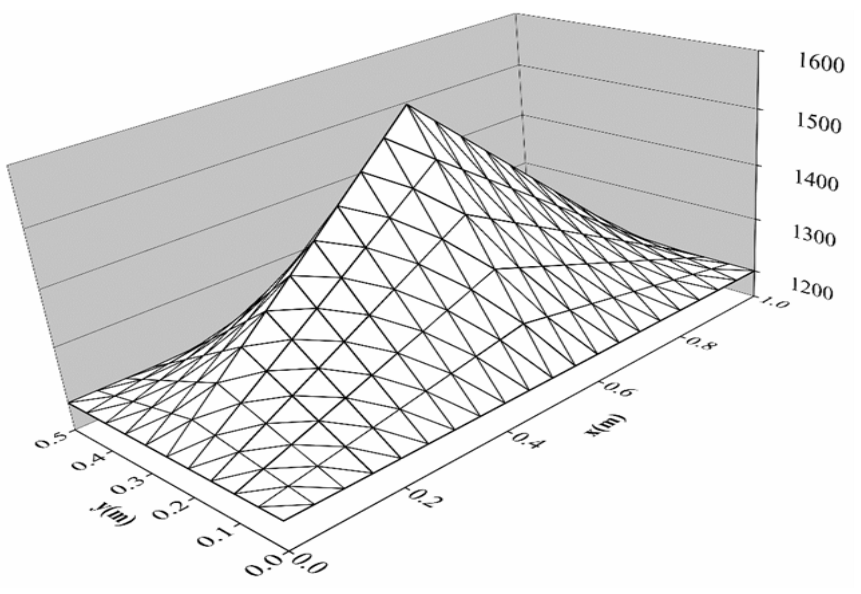

(a)
Table 8. Temperature, mole fractions of $\mathrm{H}_{2} \mathrm{O}$ and $\mathrm{CO}_{2}$ used in the 2D cases. ${ }^{[94,145]}$

\begin{tabular}{lllll}
\hline Case & $\begin{array}{l}\text { gas } \\
\text { species }\end{array}$ & Temperature $(\mathrm{K})$ & Mole fraction & \\
\hline 14 & $\mathrm{CO}_{2}$ & isothermal: 1000 & homogeneous: 0.1 & \\
15 & $\mathrm{CO}_{2}$ & $\begin{array}{l}\text { non-isothermal: } \\
\text { Eq. (91) }\end{array}$ & $\begin{array}{l}\text { inhomogeneous: } \\
(91)\end{array}$ & Eq. \\
& & isothermal: 1000 & homogeneous: 0.2 & \\
16 & $\mathrm{H}_{2} \mathrm{O}$ & non-isothermal: & inhomogeneous: & Eq. \\
17 & $\mathrm{H}_{2} \mathrm{O}$ & Eq. (91) & $(91)$ & \\
& & non-isothermal: & homogeneous: & \\
18 & $\mathrm{H}_{2} \mathrm{O}+\mathrm{CO}_{2}$ & Eq. (93) & $0.2 \mathrm{H}_{2} \mathrm{O}+0.1 \mathrm{CO}_{2}$ & \\
& & & &
\end{tabular}

In Case 14, an isothermal homogeneous medium filled with pure $\mathrm{CO}_{2}$ was investigated with the temperature $1000 \mathrm{~K}$ and $\mathrm{CO}_{2}$ mole fraction 0.1. Fig. 30 shows the radiative sources along the centerline in the $x$ and $y$ direction calculated by the LBL, SNB, SNBCK, WSGG and SLW models. The LBL results were taken from by Chu et al. ${ }^{[94]}$ based on HITEMP 2010. The SNB results were calculated using the dataset of Soufiani and Taine. ${ }^{\left[{ }^{[6]}\right.}$ Calculations of the SNBCK model were conducted also using the data of Soufiani and Taine. ${ }^{[56]}$ The seven-point Gausse Lobatto quadrature was used in the SNBCK model as well. The weighting factors of the WSGG model in Case 14 were provided by Smith $e t$. al. ${ }^{[70]}$ In the SLW model, 15 cross-sections logarithmically spaced from $3 \times 10^{-5}$ to $600 \mathrm{~m}^{2} / \mathrm{mol}$ for $\mathrm{CO}_{2}$ and from $3 \times 10^{-5}$ to $60 \mathrm{~m}^{2} / \mathrm{mol}$ for $\mathrm{H}_{2} \mathrm{O}$ were used. ${ }^{[76]}$ The SNB and SNBCK predictions were in excellent agreement with the LBL predictions. The difference of results between SNB and SNBCK was small because the same SNB parameters were used. The average relative errors of WSGG and SLW were about 3 times of those of SNB and SNBCK as shown in Fig. 30(c).

In Case 15, the medium is a non-isothermal and inhomogeneous, filled with $\mathrm{CO}_{2}$ at the temperature distribution as shown in Fig. 29(a). Fig. 31 shows that the

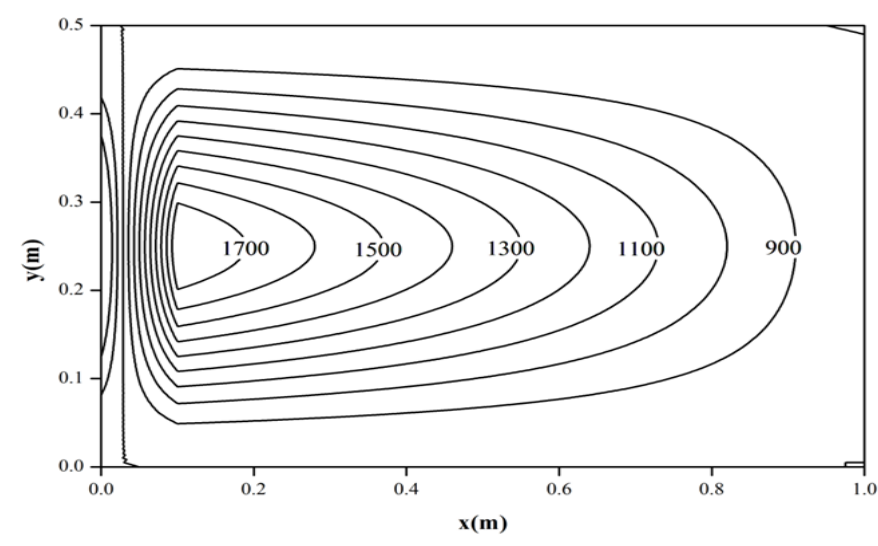

(b)

Fig. 29 The temperature distributions in (a) Case 15 and 17, and (b) Case 18. (Reproduced with the permission form [145]. Copyright@1999 Elsevier Science B.V.) 


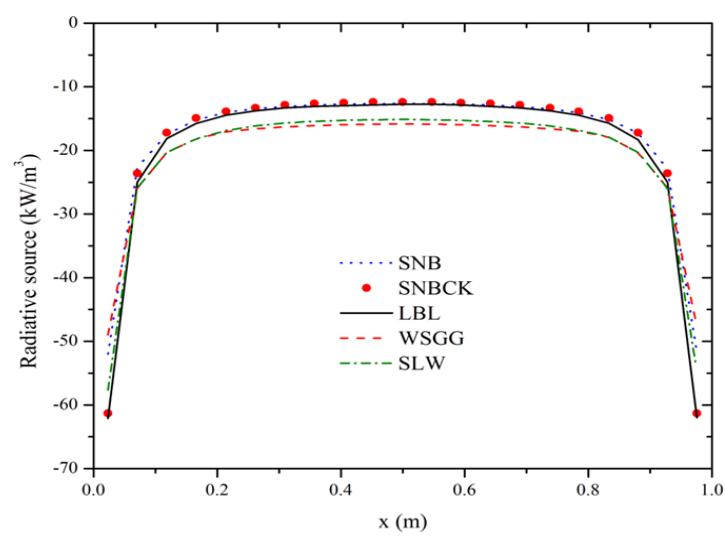

(a)

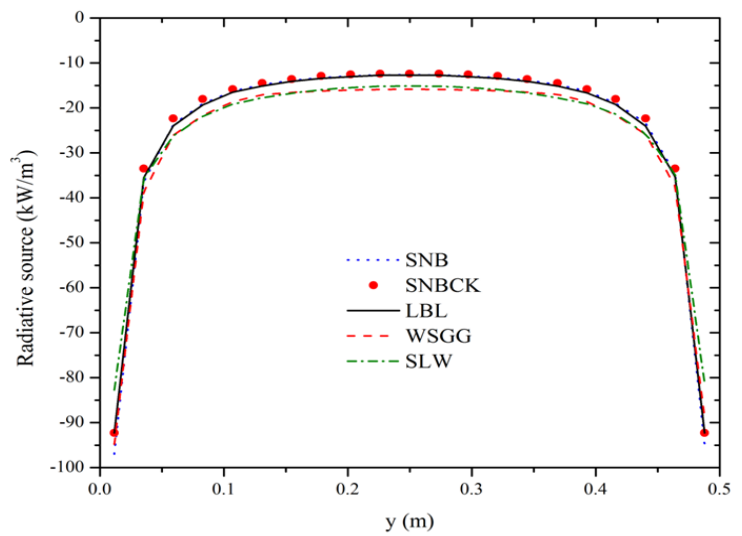

(b)

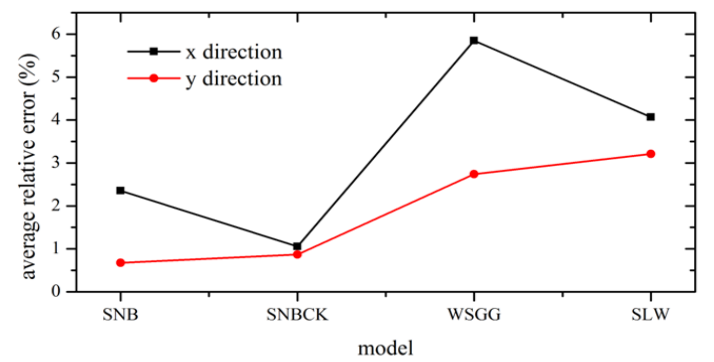

(c)

Fig. 30 Distributions of the radiative sources along the enclosure centerline in the (a) $x$ direction, (b) $y$ direction and (c) average relative error for Case 14.

maximum radiative source appears at the central coordinate point due to the highest temperature at the center of the enclosure. The predictions of SNB and SNBCK had better

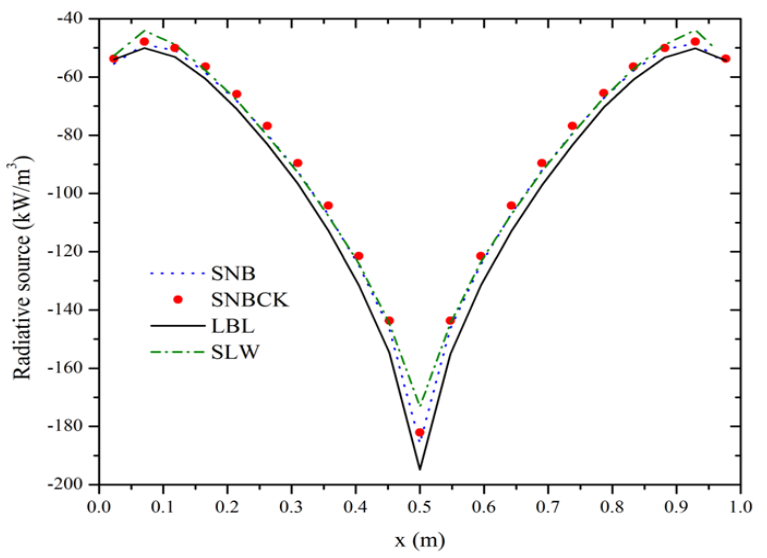

(a) performance than that of SLW at the borders and the center, but the SLW performed better than SNB and SNBCK at other grids.

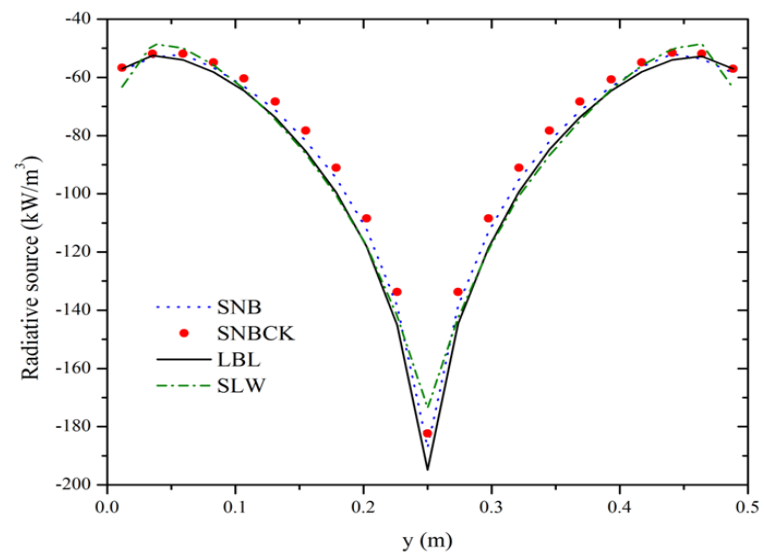

(b)

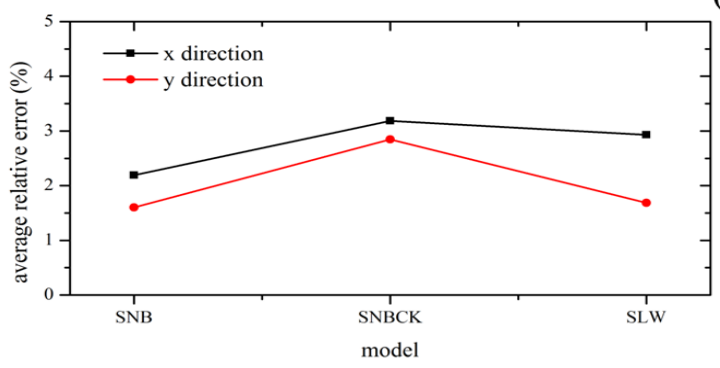

(c)

Fig. 31 Distributions of the radiative sources along the enclosure centerline in (a) $x$ direction, (b) $y$ direction and (c) average relative errors for Case 15. 


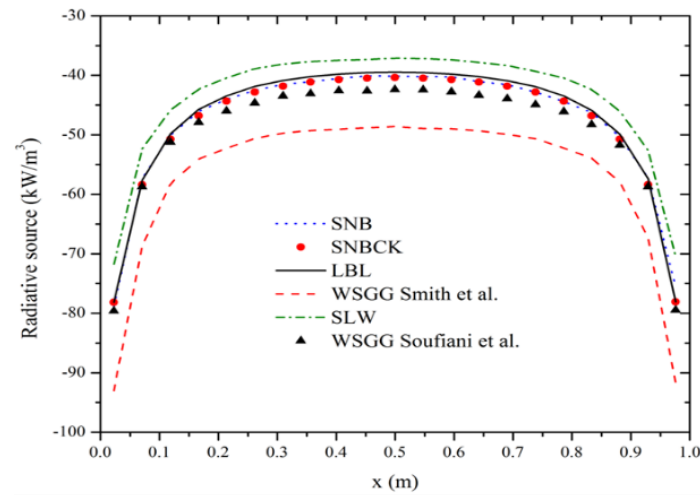

(a)

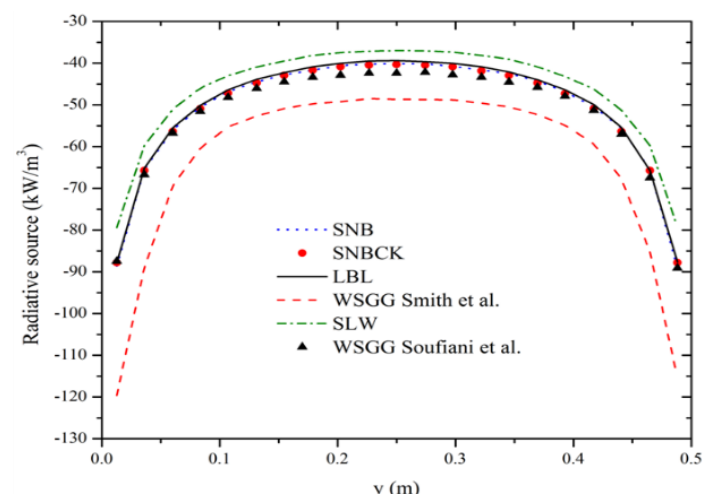

(b)

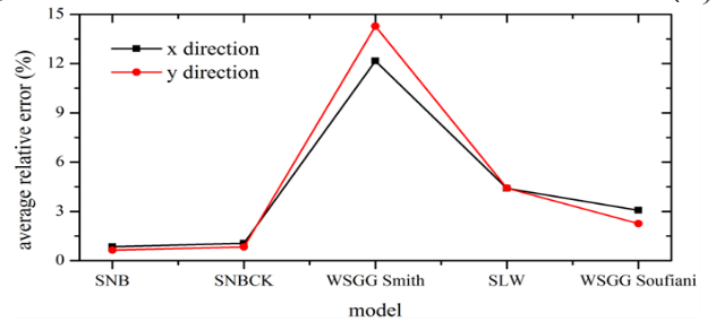

(c)

Fig. 32 Distributions of the radiative sources along the enclosure centerline in (a) $x$ direction, (b) $y$ direction and (c) average relative errors for Case 16.

$\mathrm{H}_{2} \mathrm{O}$ was added in the isothermal homogeneous medium of that for $\mathrm{CO}_{2}$ in Case 14. Nonetheless, the errors of SLW and Case 16. The WSGG model calculated by the weighting WSGG-Smith models in Fig. 32(c) were larger than those in factors developed by Soufiani et al. ${ }^{[184]}$ were added in the Fig. Fig.30(c), indicating that the $\mathrm{H}_{2} \mathrm{O}$ parameters in SLW 32 for comparisons. The results show that the SNB, SNBCK developed by Ref. ${ }^{[76]}$ and WSGG developed by Smith et al. ${ }^{[70]}$ and WSGG-Soufiani predictions were in good agreement with were not suitable for isothermal homogeneous 2D cases. In the LBL predictions, while SLW overpredicted the radiative Case 17, the medium is non-isothermal and inhomogeneous, sources and WSGG-Smith underpredicted the radiative filled with $\mathrm{H}_{2} \mathrm{O}$ at the temperature distribution as shown in Fig. sources. The accuracy of the SNB and SNBCK models for 29(a). As expected, the radiative source reached the lowest $\mathrm{H}_{2} \mathrm{O}$ in the isothermal homogeneous medium was similar to value in the center of the enclosure due to the strong radiative

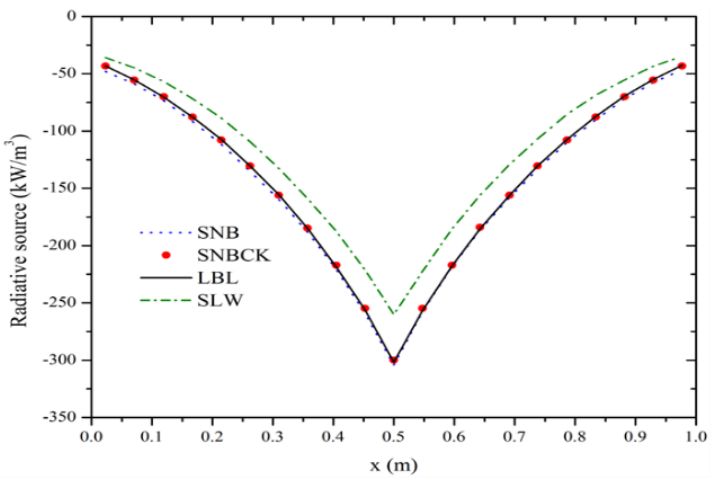

(a)

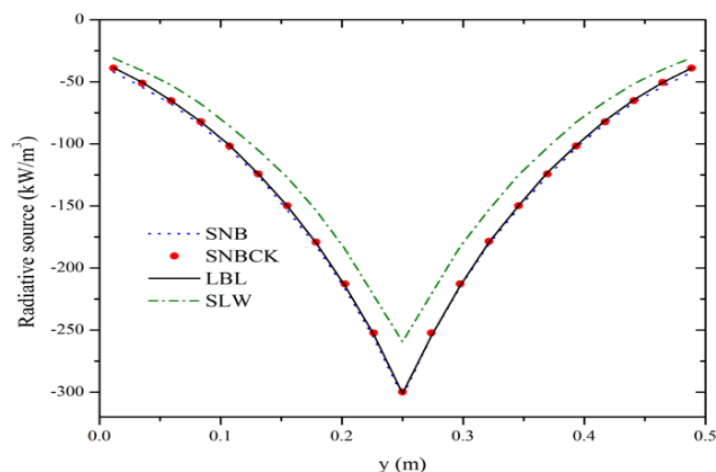

(b)

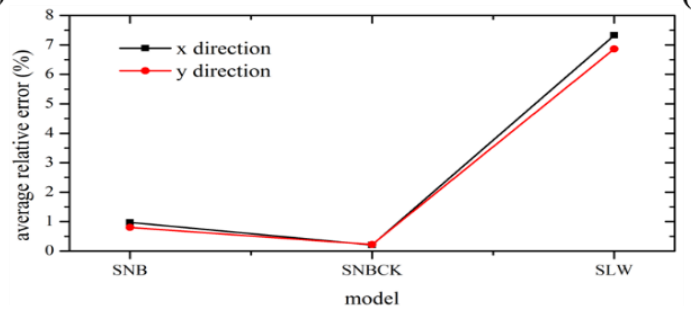

(c)

Fig. 33 Distributions of the radiative sources along the enclosure centerline in (a) $x$ direction, (b) $y$ direction and (c) average relative errors for Case 17. 


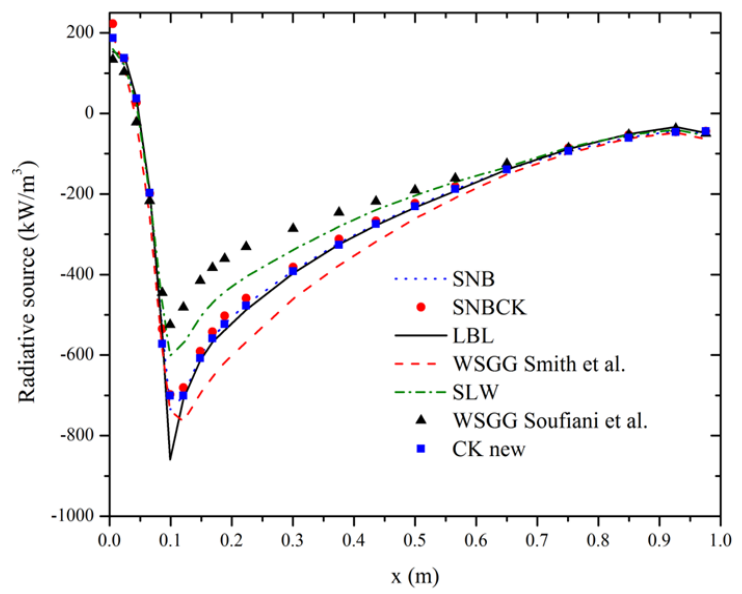

(a)

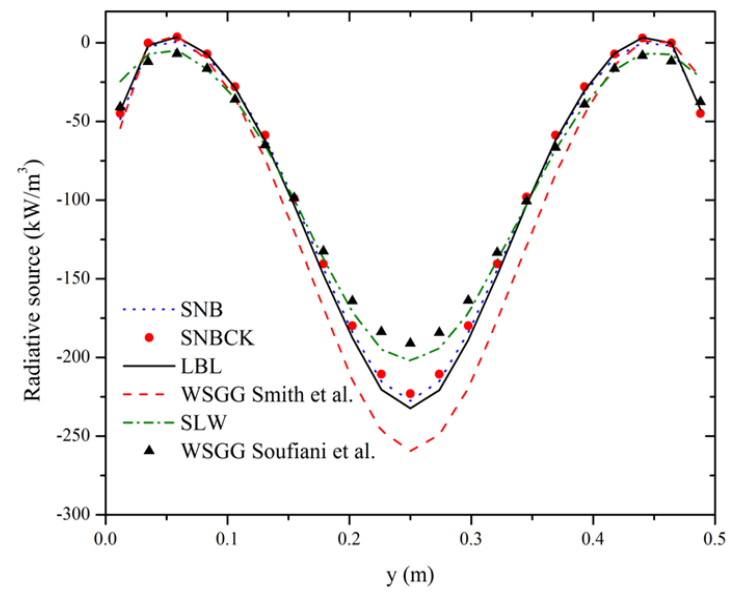

(b)

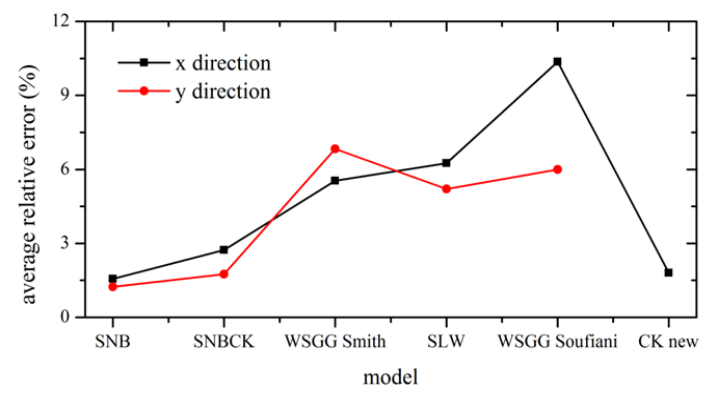

(c)

Fig. 34 Distributions of the radiative sources along the enclosure centerline in (a) $x$ direction, (b) $y$ direction and (c) average relative errors for Case 18.

emission. The SNB and SNBCK predictions were in excellent agreement with the LBL predictions, while the SLW overpredicted the radiative source in the non-isothermal and inhomogeneous case for $\mathrm{H}_{2} \mathrm{O}$. The errors of SLW (7.3\%) in Fig. 33(c) are even larger than those in Fig. 32 (c) (4.4\%). Therefore, the $\mathrm{H}_{2} \mathrm{O}$ parameters in SLW developed by Denison and $\mathrm{Webb}^{[76]}$ are not suitable for non-isothermal and inhomogeneous cases.

In Case 18, a non-isothermal homogeneous medium filled with $\mathrm{H}_{2} \mathrm{O}$ and $\mathrm{CO}_{2}$ gas mixture was investigated. Distributions of temperature are given in Fig. 29(b) and gas mole fractions of $\mathrm{H}_{2} \mathrm{O}$ and $\mathrm{CO}_{2}$ were 0.2 and 0.1 , respectively. The SNB and SNBCK results were calculated using the parameters derived by Soufiani and Taine ${ }^{[56]}$ from the EM2C spectroscopic database based on HITRAN1992 with hot lines added and approximate theoretical and empirical formulas. The new CK results were calculated by Amiri et al. ${ }^{[185]}$ using the updated SNB parameters developed by Riviere and Soufiani ${ }^{[57]}$ based on HITEMP 2010. As shown in Fig. 34(a), the SNB, SNBCK and $\mathrm{CK}$ new predictions were in good agreement with the LBL predictions, while SLW and WSGG-Soufiani overpredicted the radiative sources and WSGG-Smith underpredicted the radiative sources. Fig. 34 (c) shows that the errors of new CK $(1.8 \%)$ is less than that of $\operatorname{SNBCK}(2.7 \%)$ in the $x$ direction because HITEMP 2010 used in new CK model was the same as the LBL.
Table 9. Temperature, mole fractions of $\mathrm{H}_{2} \mathrm{O}$ and $\mathrm{CO}_{2}$ used in the 3D cases. ${ }^{[186]}$

\begin{tabular}{|c|c|c|c|}
\hline Case & $\begin{array}{l}\text { gas } \\
\text { species }\end{array}$ & Temperature $(\mathrm{K})$ & Mole fraction \\
\hline 19 & $\mathrm{H}_{2} \mathrm{O}$ & isothermal: 1000 & homogeneous: 1.0 \\
\hline 20 & $\mathrm{H}_{2} \mathrm{O}$ & isothermal: 1000 & $\begin{array}{l}\text { inhomogeneous: Eq. } \\
(94)\end{array}$ \\
\hline 21 & $\mathrm{H}_{2} \mathrm{O}, \mathrm{CO}_{2}$ & $\begin{array}{l}\text { Non-isothermal: Eq. } \\
\text { (95) }\end{array}$ & homogeneous: $0.2,0.1$ \\
\hline
\end{tabular}

\section{$5.33 \mathrm{D}$ cases}

The geometry of all the investigated $3 \mathrm{D}$ cases is a rectangular enclosure of $2 \mathrm{~m} \times 2 \mathrm{~m} \times 4 \mathrm{~m}$. All the surrounding walls are black and constant at a temperature of $300 \mathrm{~K}$. The pressure of the gas mixture in the enclosure is kept at $1 \mathrm{~atm}$ for all the cases. Three cases were studied and the distributions of temperature and species mole fraction are summarized in Table 9.

For Case 20, the distribution of mole fraction of $\mathrm{H}_{2} \mathrm{O}$ was described as

$$
X_{\mathrm{H}_{2} \mathrm{O}}=4\left(z / L_{z}\right)\left(1-z / L_{z}\right)
$$

where $L_{z}=4 \mathrm{~m}$ is the height of the enclosure.

For the non-isothermal Case 21, the temperature was given as follows

$$
\begin{gathered}
T=\left(T_{c}-T_{e}\right) f(r / R)+T_{e} \\
f(r / R)=1-3(r / R)^{2}+2(r / R)^{3}
\end{gathered}
$$




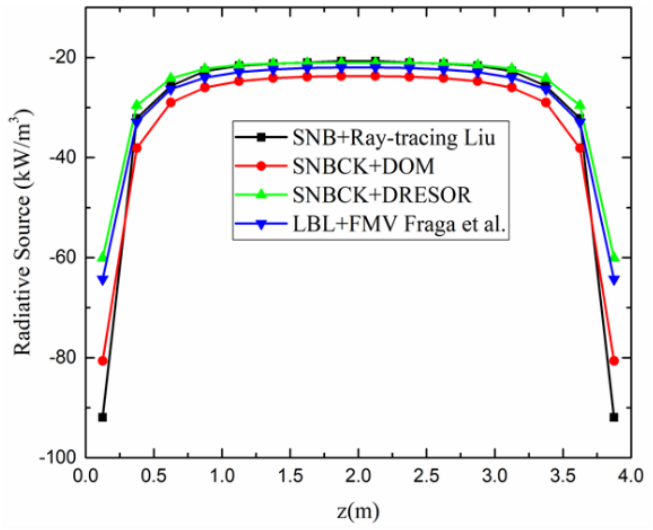

(a)

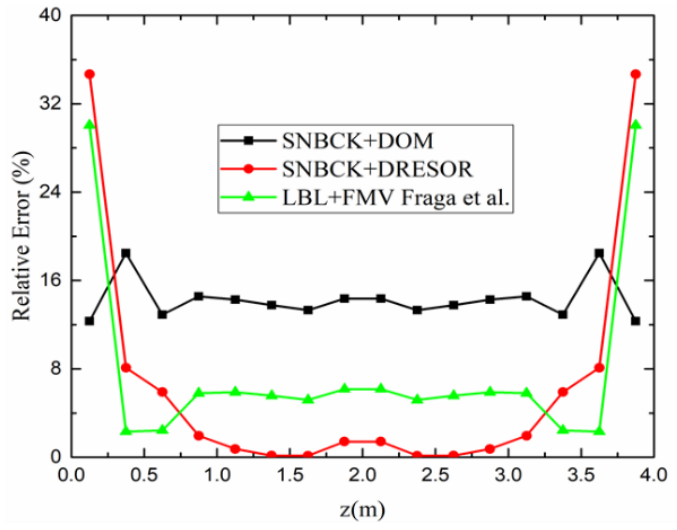

(b)

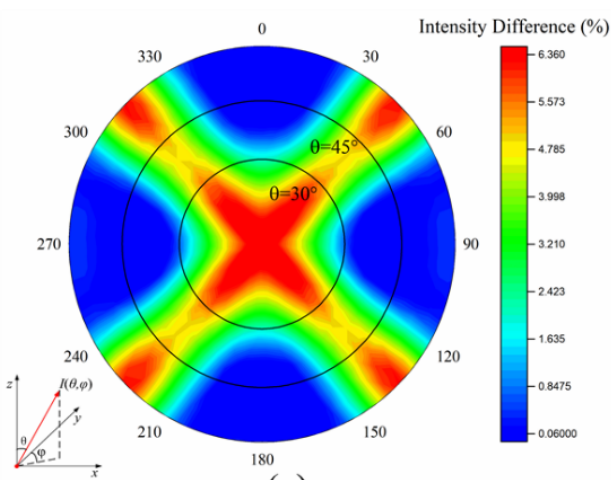

(c)

Fig. 35 Results of Case 19: (a) distribution of radiative source along the enclosure centerline; (b) radiative errors of the radiative source $\left|\left(Q-Q_{\mathrm{SNB}}\right) /\left(Q_{\mathrm{SNB}}\right)_{\mathrm{MAX}}\right|$; (c) radiative intensity difference located in the center of the top wall $\left|\left(I_{\mathrm{DOM}}-I_{\mathrm{DRESOR}}\right) / I_{\mathrm{DOM}}\right|$, concentric circles represent zenith angles $(\theta)$ and the numbers in the outermost circle represent azimuth angles $(\varphi)$. (Reproduced with the permission from [187]. Copyright@2020 Elsevier Masson SAS)

where $T_{\mathrm{e}}=800 \mathrm{~K}$ is the exit $(z=4.0 \mathrm{~m})$ temperature. $T_{\mathrm{c}}$ is the temperature along the centerline and is assumed to increase linearly from $400 \mathrm{~K}$ at $z=0 \mathrm{~m}$ to $1800 \mathrm{~K}$ at $z=0.375 \mathrm{~m}$ and decrease linearly to $800 \mathrm{~K}$ at $z=4.0 \mathrm{~m} . R=1 \mathrm{~m}$ and $r$ is the distance from the enclosure centerline. The gas temperature outside the circular region is assumed to be uniform at $800 \mathrm{~K}$.

In Case 19, an isothermal homogeneous medium filled with $\mathrm{H}_{2} \mathrm{O}$ was investigated. The SNB results were calculated using the parameters derived by Soufiani and Taine ${ }^{[5]}$ from the EM2C spectroscopic database based on HITRAN 1992 with hot lines added and approximate theoretical and empirical formulas. The SNBCK and the LBL results were calculated via the HITEMP 2010 database. The results obtained by Raytracing with SNB parameters ${ }^{[186]}$ served as a basis of comparison for the evaluation of accuracy of other gas radiation models. As shown in Fig. 35(a), the radiative sources calculated by DRESOR were in good agreement with those calculated by Ray-tracing, except the region close to the walls. On the contrary, DOM and FMV ${ }^{[188]}$ underpredicted the radiative sources. Fig. 35(b) shows that in the region of 0.1$0.3 \mathrm{~m}$ along the centerline, the relative errors of DRESOR $(<$ $2 \%)$ were less than those of FVM ( 6\%) and DOM ( 14\%). The differences between the radiative intensities calculated by DOM and DRESOR using $\mathrm{T}_{4}\left(128\right.$ directions) ${ }^{[189]}$ sets are shown in Fig. 35(c). The concentric circles represent zenith angles $(\theta)$ and the numbers in the outermost circle represent azimuth angles $(\varphi)$. As shown in Fig. 35(c), the maximum intensity difference was $6.36 \%$. The intensity differences in azimuth $45^{\circ}, 135^{\circ}, 225^{\circ}$ and $315^{\circ}$ were over $5 \%$ larger than the other directions. Because the optical thickness of the medium in these directions were larger and radiation emits from the medium were relatively strong.

An isothermal and non-homogenous medium filled with pure $\mathrm{H}_{2} \mathrm{O}$ was considered in Case 20. The gas parameters of SNB, SNBCK and LBL were conducted as in Case 19. As shown in Fig. 36(a), comparing to the Ray-tracing results, the DOM and FMV results underpredicted the radiative sources along the centerline. While, in the region close to the walls, the DRESOR results were smaller than the Ray-trancing results. Fig. 36(b) shows that the relative errors close to the walls were higher than those in the center region due to the drastic differences of temperature between the gas and the walls. In Fig. 36(c), the radiative intensity differences in azimuth $45^{\circ}, 135^{\circ}, 225^{\circ}$ and $315^{\circ}$ were obvious larger than the other directions. However, the maximum intensity difference was $11.4 \%$ in case 20 which was larger than that in case 19 . That indicated a larger intensity difference would be observed when an inhomogeneous medium was considered in calculation. 


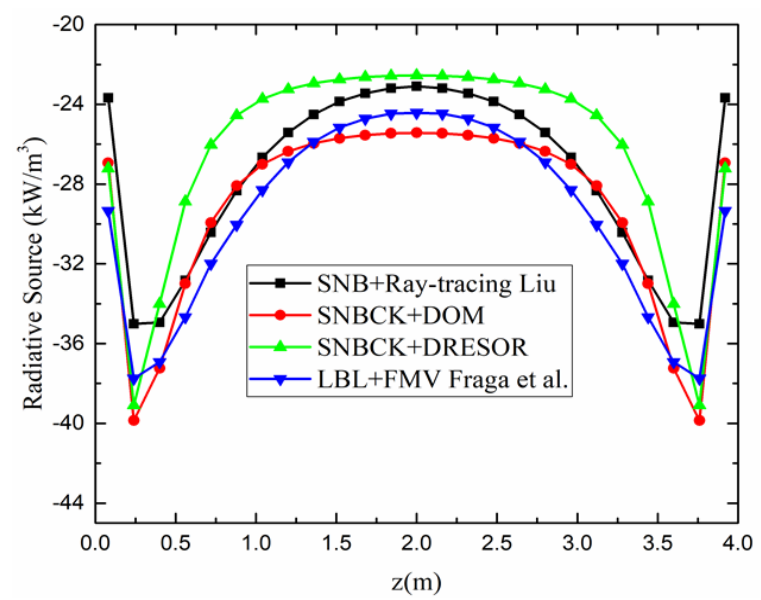

(a)

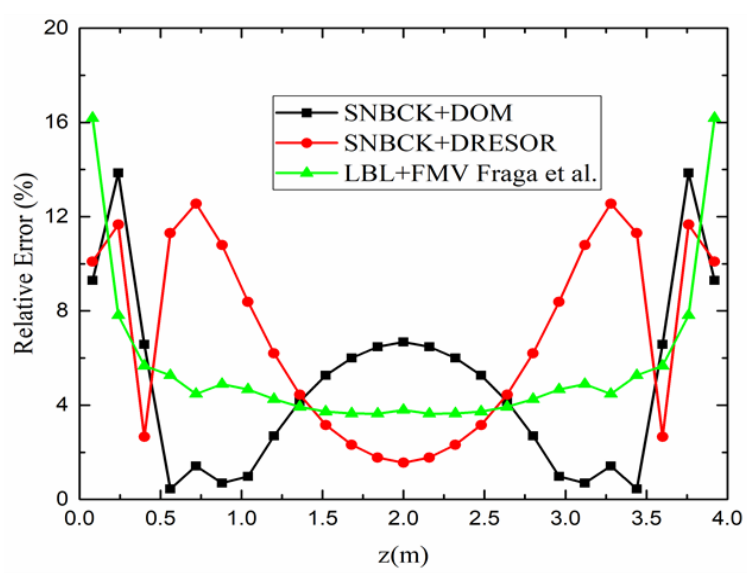

(b)

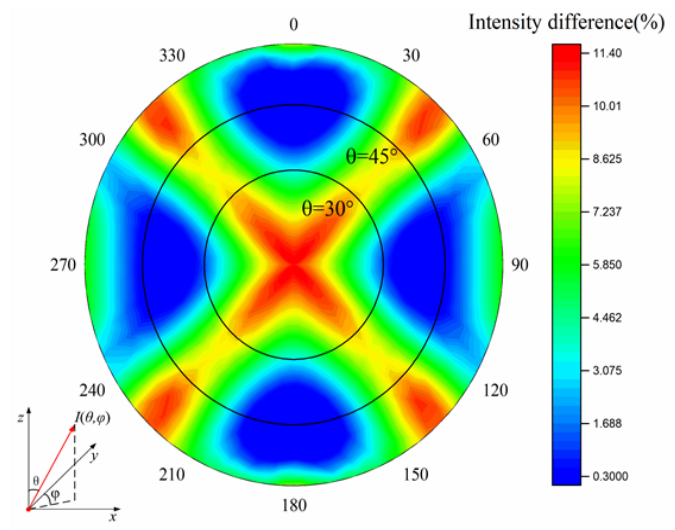

(c)

Fig. 36 Results of Case 20: (a) distribution of radiative source along the enclosure centerline; (b) radiative errors of the radiative source $\left|\left(Q-Q_{\mathrm{SNB}}\right) /\left(Q_{\mathrm{SNB}}\right)_{\mathrm{MAX}}\right|$; (c) radiative intensity difference located in the center of the top wall $\left|\left(I_{\mathrm{DOM}}-I_{\mathrm{DRESOR}}\right) / I_{\mathrm{DOM}}\right|$, concentric circles represent zenith angles $(\theta)$ and the numbers in the outermost circle represent azimuth angles $(\varphi)$. (Reproduced with the permission from [187]. Copyright@2020 Elsevier Masson SAS)

In Case 21, a three-dimensional rectangular enclosure filled with non-isothermal $\mathrm{H}_{2} \mathrm{O}$ and $\mathrm{CO}_{2}$ gases was investigated. The mole fractions of $\mathrm{H}_{2} \mathrm{O}$ and $\mathrm{CO}_{2}$ were 0.2 and 0.1 respectively. In Fig. 37(a), the DOM results were in good agreement with the Ray-tracing results. Comparing to the Raytracing results, DRESOR slightly overestimated the radiative sources while the FMV resulted in a little underestimation. Fig. 37(b) shows that the relative errors of DOM, DRESOR and FMV were over $5 \%$ near the position $z=0.375 \mathrm{~m}$ along the enclosure centerline because the radiative source here was relative stronger. However, the relative errors of DOM, DRESOR and FMV were under 5\% along the centerline $z=0.5-4.0 \mathrm{~m}$ due to a slower medium temperature change. Fig. 37 (c) shows the radiative intensity differences in azimuth $0^{\circ}$, $90^{\circ}, 180^{\circ}$ and $270^{\circ}$ were over $5 \%$ and under $5 \%$ in other azimuths. Though a non-isothermal medium was calculated in this case, the maximum intensity difference $(6.98 \%)$ was close to that in case $19(6.36 \%)$ whose medium was isothermal. That was because mole fractions of medium in this case were lower than that in case 19.

As manifested by Cases 19, 20 and 21, the radiative intensity in any angle calculated by DRESOR and DOM methods were similar. Nevertheless, what researchers are also interested in is the computing efficiency of the RTE method. The effect of different directional resolutions on the computing time was examined with Case 19 by performing the calculations using the $\mathrm{T}_{4}$ (128 directions), $\mathrm{T}_{6}$ (288 directions), $\mathrm{T}_{8}$ (512 directions) and $\mathrm{T}_{10}$ (800 directions) sets. As shown in Fig. 38, with increasing number of directions, the computation time of the DOM method increased faster than that of the DRESOR method. The computation time of DOM was less than half of that of DRESOR with $\mathrm{T}_{4}$ sets. In comparison, when the number of directions exceeds 288 , computation time of DOM became progressively higher than that of DRESOR.

\subsection{Machine learning}

Among various models, the WSGG, SLW and FSK models have drawn the most recent attention due to their efficiency, accuracy and compatibility with the common RTE solvers. These three models rely on some functions or database to calculate the gray gas absorption coefficients depending on the pressure, temperature and species molar concentrations. In the WSGG model, the gray gas absorption coefficients can be directly calculated from polynomial functions, which makes it 


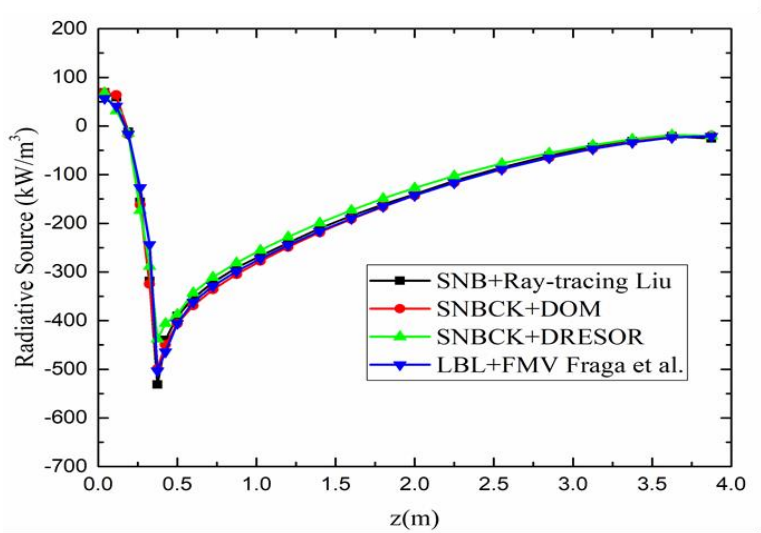

(a)

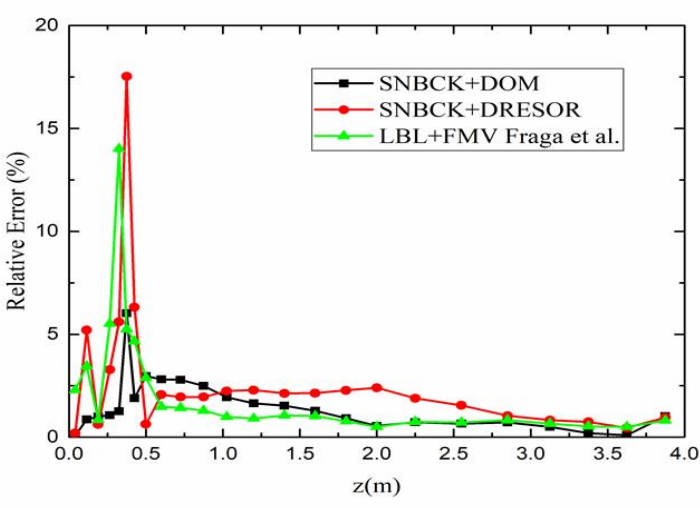

(b)

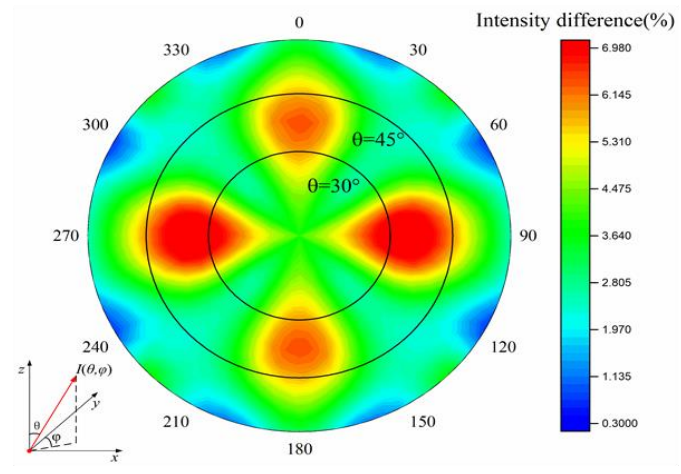

(c)

Fig. 37 Results of Case 21: (a) distribution of radiative source along the enclosure centerline; (b) radiative errors of the radiative source $\left|\left(Q-Q_{\mathrm{SNB}}\right) /\left(Q_{\mathrm{SNB}}\right)_{\mathrm{MAX}}\right|$; (c) radiative intensity difference located in the center of the top wall $\left|\left(I_{\mathrm{DOM}}-I_{\mathrm{DRESOR}}\right) / I_{\mathrm{DOM}}\right|$, concentric circles represent zenith angles $(\theta)$ and the numbers in the outermost circle represent azimuth angles $(\varphi)$. (Reproduced with the permission from [187]. Copyright@2020 Elsevier Masson SAS)

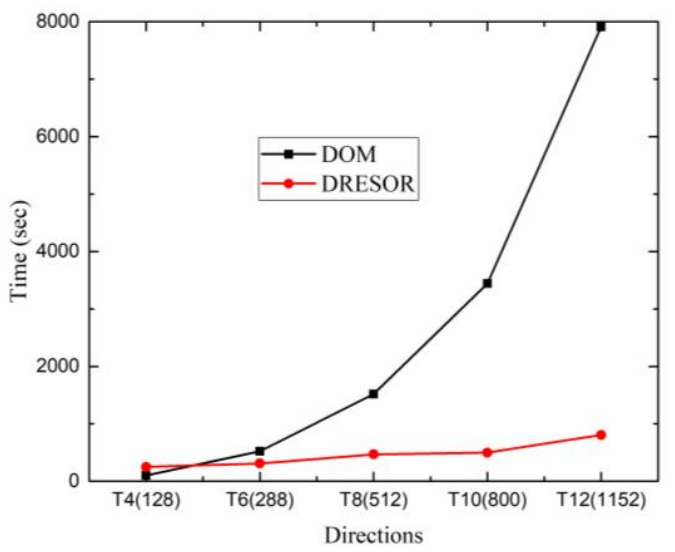

Fig. 38 Comparisons of computation time between the DOM and DRESOR methods for calculating radiative intensity with different directional sets in Case 19. (Reproduced with the permission from [187]. Copyright@2020 Elsevier Masson SAS)

very efficient. Different from the WSGG method, the SLW and FSK models need cumulative absorption coefficient functions from the database. To save computational time, these functions are pre-calculated or tabulated. Even so, they still consume a large fraction of computational time. In the SLW methods, the $F$ value can be directly calculated from the empirical functions or tables. While for non-isotherm or inhomogeneous cases, these is a need to get the absorption coefficients given a specified $\mathrm{F}$, which is not available in the functions. The common solution to this problem is the use of on-line inverse methods, such as bisection method to calculate the correlated or scaled absorption coefficients, which is very time demanding. In the FSK method, $k$ values for a set of $g$ points are tabulated for a wide range of thermophysical parameters and can be loaded before the radiation simulations, during which several additional interpolations are needed for the absorption coefficients. Although no inverse procedure is needed for FSK as in SLW, the database for FSK is very large, which somehow limits its applications. In view of the above limitations of SLW and FSK, machine learning methods are proposed to improve the efficiency.

Artificial neural network is proposed by Sun et al.$^{[190]}$ to train a surrogate model for determining cross-section from $F$ and parameters. The model is shown in Fig. 39. The training process is performed by a feed-forward neural network with two hidden layers, each with 10 neurons. The input datasets are generated by updated correlations based on HITEMP2010. Two neural network models are developed for $\mathrm{H}_{2} \mathrm{O}$ and $\mathrm{CO}_{2}$ respectively. The following figure shows the $C-F$ variations for carbon dioxide and water vapor. 
SLWog denotes the results obtained by the original SLW correlations while SLWnn by the trained neural network models. Since the cross-sections of $\mathrm{CO}_{2}$ and $\mathrm{H}_{2} \mathrm{O}$ span several orders of magnitude, the logarithmic coordinate is used. It can be seen that the inverse ALBDF predicted by the neural network model is in great agreement with that by the original SLW correlations under a specific thermal state as shown in Fig. 40. Comparisons for different thermal states can be found in Ref. ${ }^{[190]}$ Through the neural network models, the inversed ALBDF functions can be efficiently calculated, avoiding the time demanding online inverse calculations. The performance of the SLWnn model is tested for a two-dimensional pure radiation heat transfer problem. The gas temperature is uniform and wall temperature is set as $300 \mathrm{~K}$.

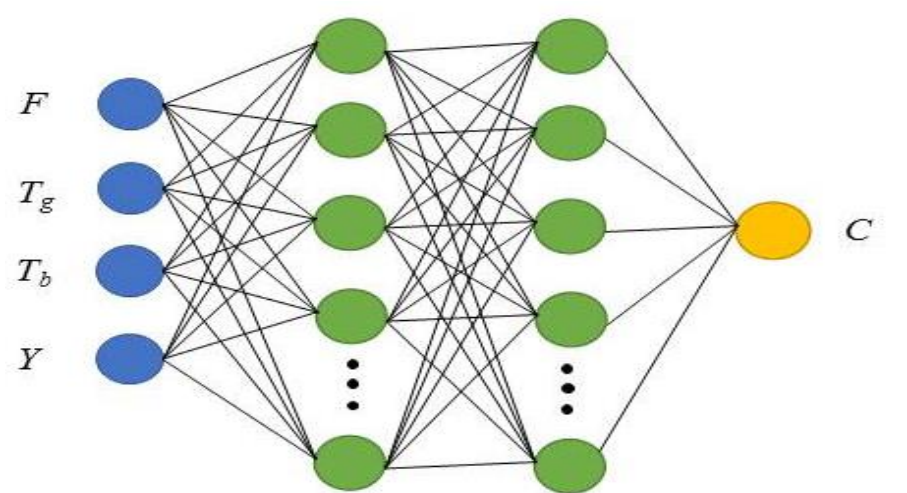

Input Hidden Output

Fig. 39 Artificial neural network for the inverse ALBDF. (Reproduced with the permission from [190]. Copyright@2019 Elsevier Ltd.)

Fig. 41 shows the radiative heat fluxes along the boundary and radiative heat sources along the center line. It can be seen

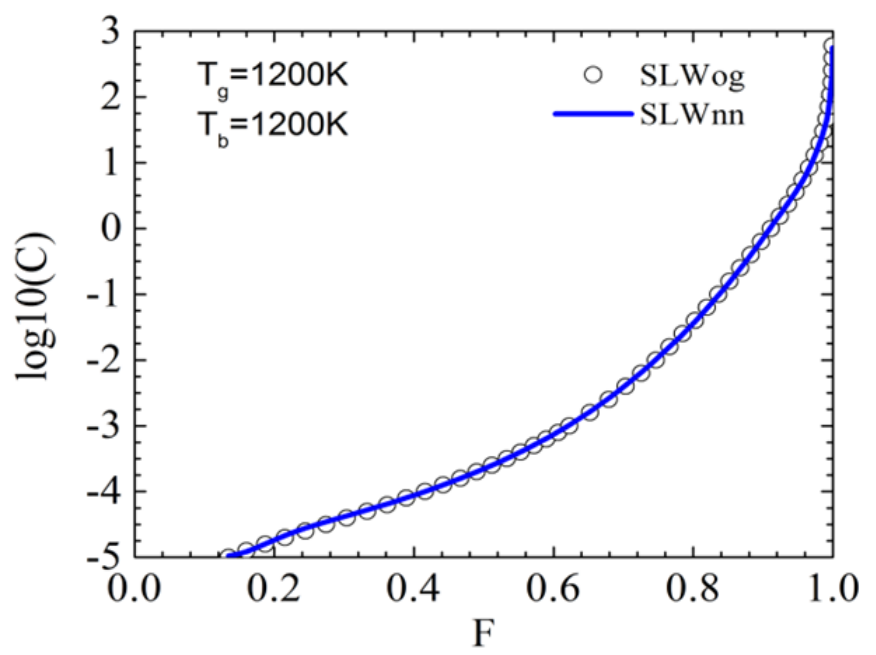

(a) that both radiative heat fluxes and heat sources predicted by the SLWnn model are very close to those by SLWog. The relative errors are around $1-2 \%$, indicating that the neural network based SLW models are accurate for non-gray gas radiation heat transfers. Table 10 compares the CPU time for the two models of the two-dimensional pure radiation heat transfer problem. For both $\mathrm{CO}_{2}$ and $\mathrm{H}_{2} \mathrm{O}$, the $\mathrm{CPU}$ time with the neural network model is only about $1 / 10$ of the original model, proving the improved efficiency of the neural network surrogate model. Moreover, the number of parameters of the SLWnn model is reduced significantly compared to the original SLW correlations.

Table 10. CPU time (s) comparisons.

\begin{tabular}{lllll} 
Grid & \multicolumn{3}{l}{ Carbon dioxide } & Water vapor \\
& SLWog & SLWnn & SLWog & SLWnn \\
$23-23$ & 8.45 & 1.25 & 8.213 & 1.288 \\
$43-43$ & 25.49 & 2.28 & 25.407 & 2.450 \\
$63-63$ & 23.46 & 4.23 & 58.23 & 4.184 \\
\hline
\end{tabular}

Neural network models have also been applied to predict the cumulative absorption coefficient functions for gas mixtures $\left(\mathrm{CO}_{2}, \mathrm{H}_{2} \mathrm{O}\right.$ and $\left.\mathrm{CO}\right)$ for the pressures range from 0.1 bar to $80 \mathrm{bar}$, temperatures range from $300 \mathrm{~K}$ to $3000 \mathrm{~K}$ and both homogeneous and nonhomogeneous media. ${ }^{[191]}$ The FSCK distribution neural network model is trained based on 32 Gauss-Chebyshev quadrature points, and the output layer has 32 neurons. Two sub-models are trained below atmospheric pressure and above atmospheric pressure respectively. 5 and 6 layers are used for the two models, and each layer has several hundred neurons. The following Fig. 42 shows comparisons of the $k$ distributions by the look-up tables and the machine learning model. Results of the two methods are almost identical and the relative errors of the Planck-mean absorption coefficient are negligible for practical applications.

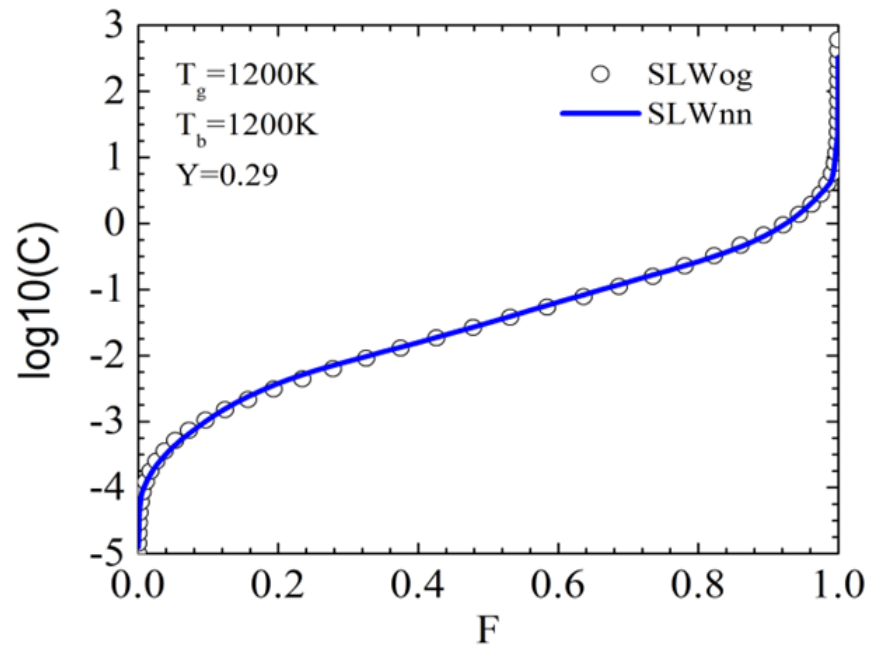

(b)

Fig. 40 Inverse ALBDFs for (a) carbon dioxide and (b) water vapor. (Reproduced with the permission from [190]. Copyright@2019 Elsevier Ltd. 

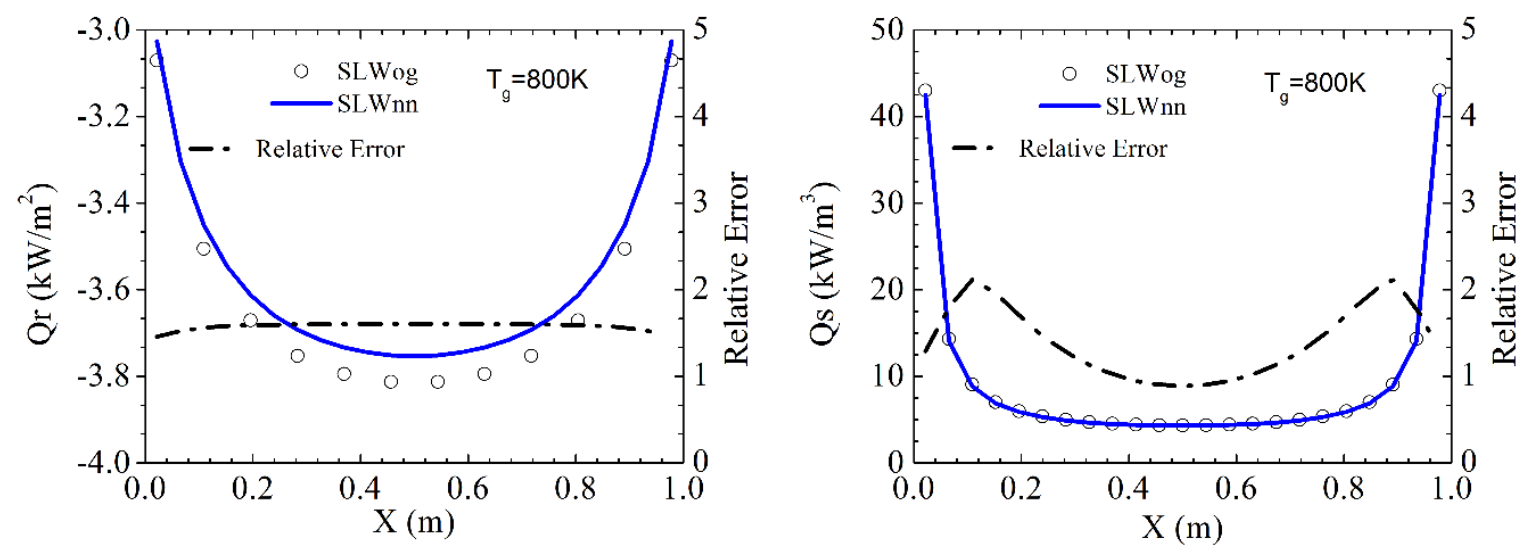

(a) carbon dioxide
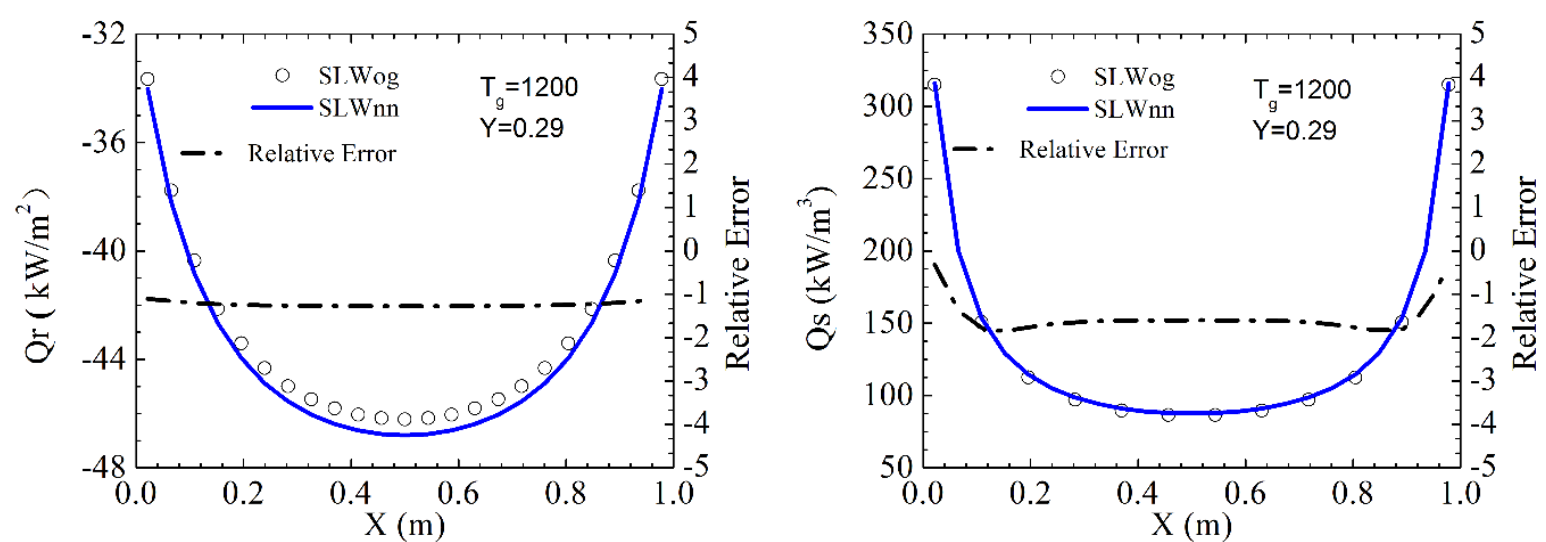

(b) water vapor

Fig. 41 Radiative heat transfer results for carbon dioxide and water vapor. (Reproduced with the permission from [190]. Copyright@2019 Elsevier Ltd.)
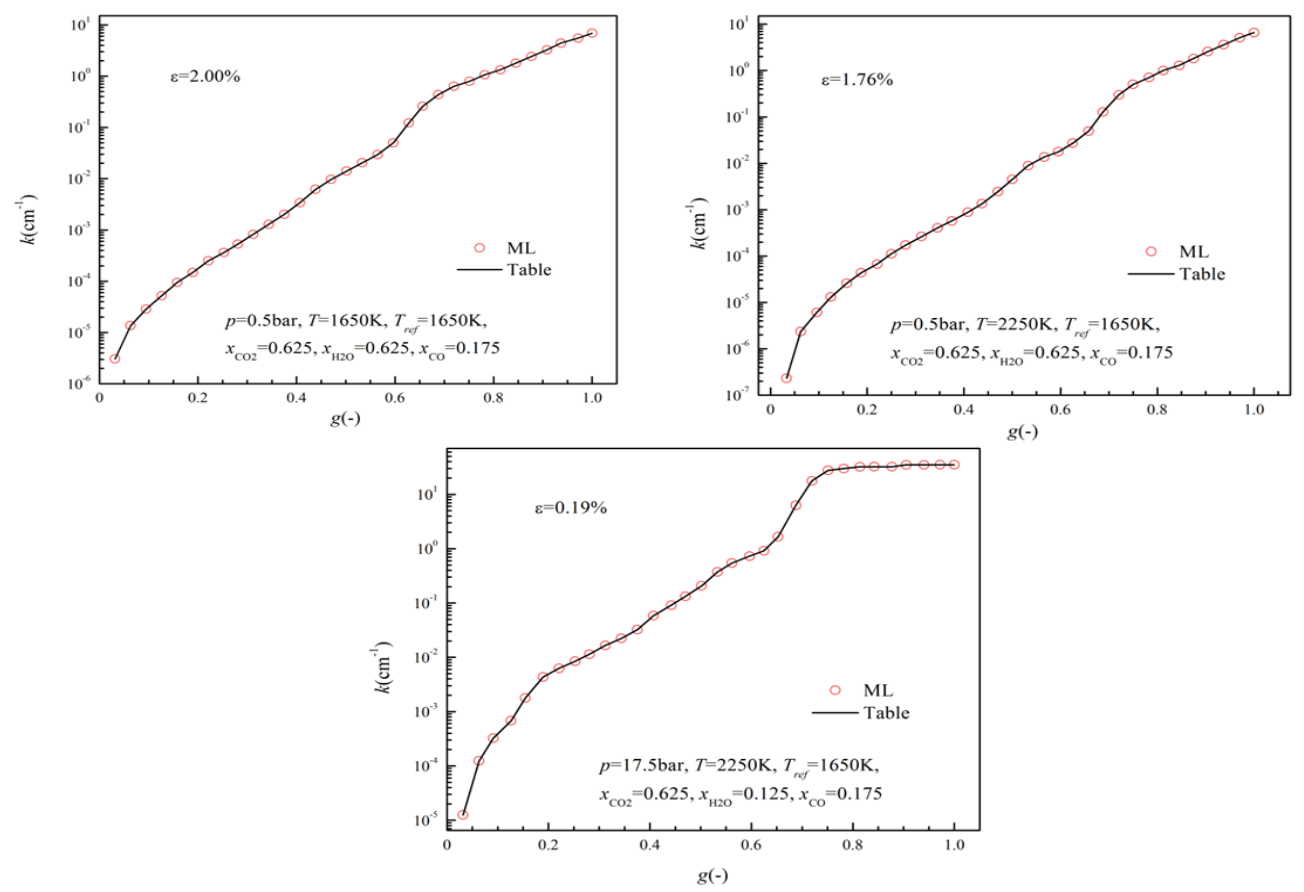

Fig. 42 FSCK distribution calculated by the neural network model. (Reproduced with the permission from [191], Copyright@2019 Elsevier Ltd.) 

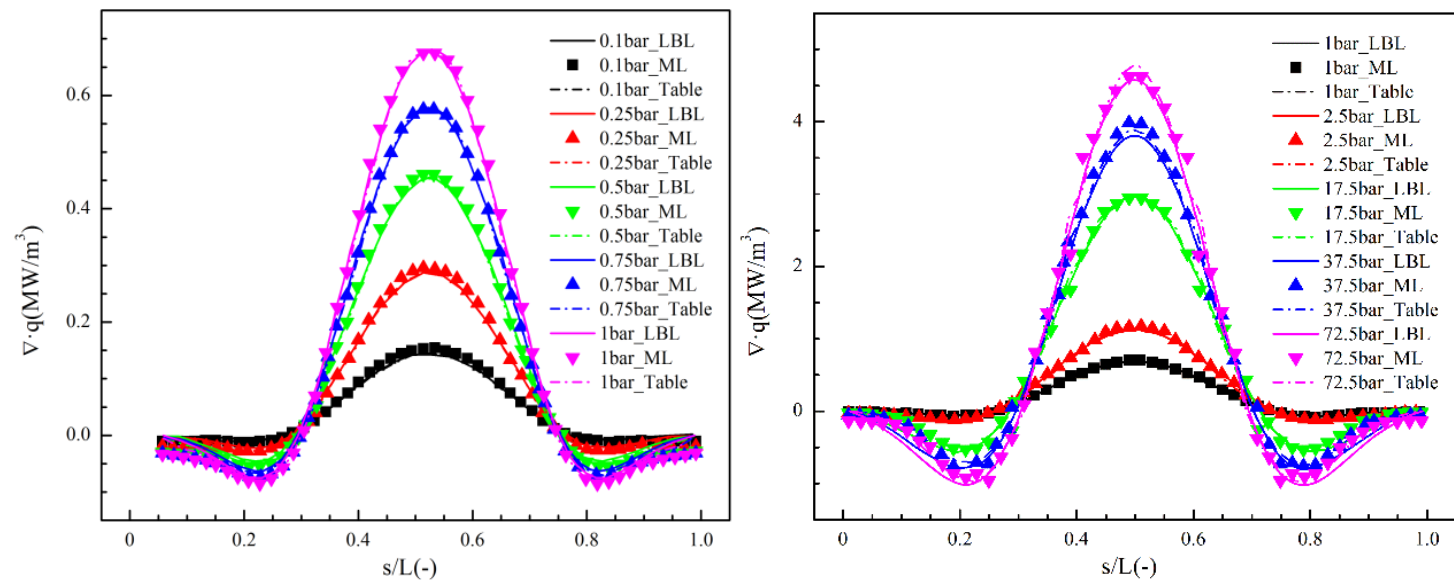

Fig. 43 Radiative heat source comparisons of LBM, FSCK table and Machine Learning model. (Reproduced with the permission from [190]. Copyright@2019 Elsevier Ltd.)

The neural network based FSCK model is also applied to a 1D non-gray gas radiation transfer problem. Fig. 43 shows the radiative heat source predicted by different models for an axisymmetric nonhomogeneous gaseous media. As indicated in the figure, the neural network trained FSCK model agrees very well with the LBL and FSCK table models, thus proving its accuracy.

As for computational efficiency, the neural network based FSCK is in the same order of magnitude with the FSCK table model, but with a significantly smaller size of database. The FSCK look-up table reaches several GB, while the neural network based FSCK model is only $35 \mathrm{MB}$, making it more convenient to use in practical combustion radiation simulations. As the model is trained in Python, its application to other programming language may induce less efficiency. More investigations on how to train an optimal FSCK model are desirable and may further improve its efficiency.

\section{Conclusions}

The research progress on the development of gas spectral line databases, gas radiation models and RTE solution methods in recent decades is systematically summarized in this paper. The performance of different gas radiation models in multidimensional system are also reviewed. The following conclusions and recommendations can be drawn.

(1) How to obtain the gas radiation characteristics efficiently and accurately in any computing environment has become the focus of the development of gas radiation models. Band models are found to be more efficient when the narrower bands are lumped, however, at the cost of accuracy because the Planck blackbody function cannot be treated satisfactorily as a constant with wide bandwidth. The FSK model is shown to be a superior model due to its high calculation efficiency.

(2) For the simulations in one-dimensional geometries, the SNB results are improved when the newest HITRAN 2016 is used due to the added hotter spectral lines. The FSK model has better accuracy comparing to WSGG in 1D non-isothermal inhomogeneous cases. The DRESOR method exhibits a better efficiency than the DOM method in the presence of wall reflection. For 1D spherical cases, P1 model performs better than DOM in pure gas environment, while DOM is more accurate than P1 for non-isotherm and inhomogeneous gas mixtures.

(3) For two-dimensional geometries, predictions obtained from the SNB and SNBCK models are in excellent agreement with the LBL predictions, while SLW and WSGG are not suitable for 2D non-isothermal and inhomogeneous cases. The new CK model in conjunction with the updated SNB parameters developed by Riviere and Soufiani ${ }^{[57]}$ leads to improved results than the old SNBCK model using the outdated SNB parameters developed by Soufiani and Taine. ${ }^{[56]}$ (4) For three-dimensional cases, the SNB results are in good agreement with the LBL results. The SNBCK results using the DRESOR method perform closely to those using the DOM method in all the reviewed cases. Moreover, once the number of discrete directions of radiative intensity is over T6, the computational efficiency of DRESOR method is markedly higher than that of the DOM method.

(5) In addition to the aforementioned classical methods and models, the emerging machine learning technologies are proposed to solve gas radiation problems. Artificial neural network has been applied to the SLW and FSCK models for the development of inverse ALBDF and $k$-distributions under various thermal states. The surrogate inverse ALBDF model helps to eliminate the time-consuming online inverse calculations of cross-sections from the ALBDF functions and hence improves its efficiency by nearly one order of magnitude. Machine-learning-based FSCK distributions significantly reduces size of the database, and consequently makes the method feasible and more convenient for complex applications.

\section{Acknowledgements}

SZ acknowledges the National Key Research Development Program of China (No.2017YFB0601900), the National Natural Science Foundation of China (No. 51976057, 51922040 and 51827808) and the Fundamental Research Funds for the Central Universities (No. 2020DF01). 


\section{Supporting information}

Not Applicable.

\section{Conflict of interest}

There are no conflicts to declare.

\section{References}

[1] R. Viskanta, Int. J. Eng. Sci., 19198, 36, 1677-1699, doi: 10.1016/S0020-7225(98)00053-6.

[2] S. Lahsasni, M. Kouhila, M. Mahrouz, A. Idlimam, A. Jamali, Energy, 2004, 29, 211-224, doi: 10.1016/j.energy.2003.08.009.

[3] N. Getoff, Radiat. Phys. Chem., 2006, 75, 514-523, doi: 10.1016/j.radphyschem.2005.09.014.

[4] T. Fiala, T. Sattelmayer, S. Gröning, J. Hardi, R. Stützer, S. Webster, M. Oschwald, J. Propul. Power, 2017, 33, 490-500, doi: 10.2514/1.B36280.

[5] Y.S. Sim, S.-O. Kim, H.W. Myung, E.-K. Kim, Nucl. Eng. Des., 2000, 199, 167-186, doi: 10.1016/S0029-5493(99)00067-9. [6] A. Roychowdhury, J. Srinivasan, Heat Mass Tran., 1994, 30, 71-75, doi: 10.1007/BF00715012.

[7] T. Qiu, C. Tien, Int. J. Heat Mass Tran., 1992, 35, 719-726, doi: 10.1016/0017-9310(92)90131-B.

[8] F. Liu, Y. Ai, W. Kong, Int. J. Hydrogen Energ., 2014, 39, 3936-3946, doi: 10.1016/j.ijhydene.2013.12.151.

[9] D. Ning, A. Fan, H. Yao, Int. J. Hydrogen Energ., 2017, 42, 22015-22026, doi: 10.1016/j.ijhydene.2017.07.114.

[10] P. Qian, M. Liu, X. Li, F. Xie, Z. Huang, C. Luo, X. Zhu, Int. J. Hydrogen Energ., 2020, 45, 5002-5013, doi: 10.1016/j.ijhydene.2019.12.094.

[11] S. Zheng, R. Sui, W. Liang, H. Zhou, C.K. Law, Combust. Flame, 2020, 221, 86-93, doi: 10.1016/j.combustflame.2020.07.042.

[12] S. Zheng, Y. Yang, R. Sui, Q. Lu, Appl. Therm. Eng., 2021, 183, 116194, doi: 10.1016/j.applthermaleng.2020.116194.

[13] E.M. Sparrow, Radiation heat transfer, Routledge, 2018.

[14] W. Huang, H. Ji, J. Aerosp. Power, 2011, 26, 48-53, doi: 10.1007/s10483-011-1391-6.

[15] W. Heitler, The quantum theory of radiation, Courier Corporation, 1984.

[16] R.A. McClatchey, W. Benedict, S.A. Clough, D. Burch, R.F. Calfee, AFCRL atmospheric absorption line parameters compilation, in, Air Force Cambridge Research Labs HANSCOM AFB MA, 1973.

[17] L.S. Rothman, A. Barbe, D.C. Benner, L. Brown, C. CamyPeyret, M. Carleer, K. Chance, C. Clerbaux, V. Dana, V.M. Devi, J. Quant. Spectrosc. Ra., 2003, 82, 5-44, doi: 10.1016/S00224073(03)00146-8.

[18] L.S. Rothman, R. Gamache, R. Tipping, C. Rinsland, M. Smith, D.C. Benner, V.M. Devi, J.-M. Flaud, C. Camy-Peyret, A. Perrin, J. Quant. Spectrosc. Ra., 1992, 48, 469-507, doi: 10.1016/0022-4073(92)90115-K.

[19] L.S. Rothman, C. Rinsland, A. Goldman, S. Massie, D. Edwards, J. Flaud, A. Perrin, C. Camy-Peyret, V. Dana, J.-Y. Mandin, J. Quant. Spectrosc. Ra., 1998, 60, 665-710, doi: 10.1016/S0022-4073(98)00078-8.
[20] L. S. Rothman, R.R. Gamache, A. Goldman, L.R. Brown, R.A. Toth, H.M. Pickett, R.L. Poynter, J.-M. Flaud, C. CamyPeyret, A. Barbe, Appl. optics, 1987, 26, 4058-4097, doi: 10.1364/AO.26.004058.

[21] I.E. Gordon, L.S. Rothman, C. Hill, R.V. Kochanov, Y. Tan, P.F. Bernath, M. Birk, V. Boudon, A. Campargue, K. Chance, J. Quant. Spectrosc. Ra., 2017, 203, 3-69, doi: 10.1016/j.jqsrt.2017.06.038.

[22] L. S. Rothman, R. Wattson, R. Gamache, J.W. Schroeder, A. McCann, HITRAN HAWKS and HITEMP: high-temperature molecular database, in: Atmospheric Propagation and Remote Sensing IV, International Society for Optics and Photonics, 1995, pp. 105-111.

[23] S. Tashkun, V. Perevalov, J. Quant. Spectrosc. Ra., 2011, 112, 1403-1410, doi: 10.1016/j.jqsrt.2011.03.005.

[24] S. Tashkun, V. Perevalov, R. Gamache, J. Lamouroux, J. Quant. Spectrosc. Ra., 2015, 52, 45-73, doi: 10.1016/j.jqsrt.2014.10.017.

[25] S.A. Tashkun, V.I. Perevalov, J.-L. Teffo, A.D. Bykov, N. Lavrentieva, J. Quant. Spectrosc. Ra., 2003, 82, 165-196, doi: 10.1016/S0022-4073(03)00152-3.

[26] H. Partridge, D.W. Schwenke, J. Chem. Phys., 1997, 106, 4618-4639, doi: 10.1063/1.473987.

[27] U.G. Jørgensen, P. Jensen, G.O. Sørensen, B. Aringer, Astron. Astrophys., 2001, 372, 249-259, doi: 10.1051/00046361:20010285.

[28] R. Barber, J. Tennyson, G.J. Harris, R. Tolchenov, Mon. Not. R. Astron. Soc., 2006, 368, 1087-1094, doi: 10.1111/j.13652966.2006.10184.x.

[29] L. Rothman, I. Gordon, R. Barber, H. Dothe, R. Gamache, A. Goldman, V. Perevalov, S. Tashkun, J. Tennyson, J. Quant. Spectrosc. Ra., 2020, 111, 2139-2150, doi: 10.1016/j.jqsrt.2010.05.001.

[30] M. Alberti, R. Weber, M. Mancini, A. Fateev, S. Clausen, J. Quant. Spectrosc. Ra., 2015, 157, 14-33, doi: 10.1016/j.jqsrt.2015.01.016.

[31] H. Chu, F. Liu, H. Zhou, Int. J. Heat Mass Tran., 2011, 54, 4736-4745, doi: 10.1016/j.ijheatmasstransfer.2011.06.002.

[32] S. Zheng, W. Liang, H. Chu, H. Zhou, Fuel, 2020, 266, 117061, doi: 10.1016/j.fuel.2020.117061.

[33] J. R. Howell, M.P. Menguc, R. Siegel, Thermal radiation heat transfer, CRC press, 2010.

[34] J. Arnold, E. Whiting, G. Lyle, J. Quant. Spectrosc. Ra., 1996, 9, 775-798, doi: 10.1016/0022-4073(69)90075-2.

[35] J. Hartmann, R. L. Di Leon, J. Taine, J. Quant. Spectrosc. Ra., 1984, 32, 119-127, doi: 10.1016/0022-4073(84)90076-1.

[36] F. André, R. Vaillon, J. Quant. Spectrosc. Ra., 2007, 108, 116, doi: 10.1016/j.jqsrt.2007.02.013.

[37] H. Zhang, M.F. Modest, Int. J. Heat Mass Tran., 2003, 46, 3593-3603, doi: 10.1016/S0017-9310(03)00151-0.

[38] F. Liu, J.-L. Consalvi, P.J. Coelho, F. Andre, M. Gu, V. Solovjov, B.W. Webb, Fuel, 2020, 281, 118555, doi: 10.1016/j.fuel.2020.118555.

[39] W.M. Elsasser, Harvard Meteorological Studies, 1942, 6, 107. 
[40] S. Golden, J. Quant. Spectrosc. Ra., 1967, 7, 483-494, doi: 10.1016/0022-4073(67)90044-1.

[41] S. Golden, The Voigt analog of an Elsasser band, J. Quant. Spectrosc. Ra., 1969, 9, 1067-1081, doi: 10.1016/00224073(69)90133-2.

[42] R. Goody, Quart. J. R. Meteor. Soc., 1952, 78, 638-640, doi: 10.1002/qj.49707833820.

[43] W. Malkmus, J. Opt. Soc. A., 1967, 57, 323-329, doi: 10.1364/JOSA.57.000323.

[44] W.L. Godson, J. Meteorol. Soc. Jpn., 1955, 12, 272-284, doi: 10.1175/1520-0469(1955)012<0272:TCOITB >2.0.CO;2.

[45] C.B. Ludwig, W. Malkmus, J. E. Reardon, J.A.L. Thomson, R. Goulard, Handbook of infrared radiation from combustion gases, in, NASA Special Publication, 1973.

[46] W. L. Grosshandler, RADCAL: A Narrow-band Model for Radiation Calculations in Combustion Environment, in, 1993.

[47] A. Soufiani, J.M. Hartmann, J. Taine, J. Quant. Spectrosc. Ra., 1985, 33, 243-257, doi: 10.1016/0022-4073(85)90154-2.

[48] A. Soufiani, J. Taine, Int. J. Heat Mass Tran., 1987, 30, $437-$ 447, doi: 10.1016/0017-9310(87)90259-6.

[49] J. Taine, J. Quant. Spectrosc. Ra., 1983, 30, 371-379, doi: 10.1016/0022-4073(83)90036-5.

[50] L. Zhang, A. Soufiani, J. Taine, Int. J. Heat Mass Tran., 1988, 31, 2261-2272, doi: 10.1016/0017-9310(88)90158-5.

[51] T. Kim, J. Menart, H. Lee, J. Heat Tran., 113, 945-952, doi: 10.1115/1.2911226.

[52] T. Daguse, Study of radiative effect on laminar counterflow $\mathrm{H} 2$ /O2 /N2 diffusion flames, 2008, 106, 271-287.

[53] Y. Ju, H. Guo, F. Liu, K. Maruta, J. Fluid Mech., 1999, 379, 165-190, doi: 10.1017/S0022112098003231.

[54] S.P. Bharadwaj, M.F. Modest, Int. J. Therm. Sci., 2007, 46, 479-490, doi: 10.1016/j.ijthermalsci.2006.06.015.

[55] P. Riviere, S. Langlois, A. Soufiani, J. Taine, J. Quant. Spectrosc. Ra., 1995, 53, 221-234, doi: 10.1016/00224073(95)90009-8.

[56] A. Soufiani, J. Taine, Int. J. Heat Mass Tran., 1997, 40, $987-$ 991, doi: 10.1016/0017-9310(96)00129-9.

[57] P. Riviere, A. Soufiani, Int. J. Heat Mass Tran., 2012, 55, 3349-3358, doi: 10.1016/j.ijheatmasstransfer.2012.03.019.

[58] J. Consalvi, F. Liu, Fire Safety. J., 2015, 78, 202-218, doi: 10.1016/j.firesaf.2015.10.002.

[59] C. Qi, S. Zheng, H. Zhou, J. Quant. Spectrosc. Ra., 2017, 197, 45-50, doi: 10.1016/j.jqsrt.2016.12.021.

[60] A. A. Lacis, V. Oinas, J. Geophys. Res-Atmos., 1991, 96, 9027-9063.

[61] F. Liu, G.J. Smallwood, Ö.L. Gülder, Int. J. Heat Mass Tran., 2000, 43, 3119-3135, doi: 10.1016/S0017-9310(99)00343-9.

[62] S.S. Penner, R. Landshoff, Quantitative Molecular Spectroscopy and Gas Emissivities, 1959.

[63] D.K. Edwards, Molecular Gas Band Radiation, in: T.F. Irvine, J.P. Hartnett (Eds.) Advances in Heat Transfer, Elsevier, 1976, 115-193.

[64] F. Liu, G.J. Smallwood, O.L. Gülder, Band lumping strategy for radiation heat transfer calculations using a narrowband model,
J. Thermophys. Heat Tran., 2000, 14, 278-281, doi: 10.2514/2.6521.

[65] D.K. Edwards, W.A. Menard, Appl. Opt., 1964, 3, 621-625, doi: 10.1364/AO.3.000621.

[66] N. Lallemant, R. Weber, Int. J. Heat Mass Tran., 1996, 39, 3273-3286, doi: 10.1016/0017-9310(95)00400-9.

[67] H.C. Hottel, A. F. Sarofim, Landolt Börnstein Group VI, 1967, 142, 42-44, doi: 10.1007/10057805_11.

[68] M.K. Denison, B.W. Webb, J. Quant. Spectrosc. Ra., 1993, 50, 499-510, doi: 10.1016/0022-4073(93)90043-H.

[69] M.F. Modest, J. Quant. Spectrosc. Ra., 2003, 76, 69-83, doi: 10.1016/S0022-4073(02)00046-8.

[70] T. F. Smith, Z. F. Shen, J. N. Friedman, J. Heat Tran., 1982, 104, 602-608, doi: 10.1115/1.3245174.

[71] M. F. Modest, J. Heat Trans-T. ASME, 113, 650, doi: 10.1115/1.2910614.

[72] F. Liu, H. A. Becker, Y. Bindar, Int. J. Heat Mass Tran., 1998, 41, 3357-3371, doi: 10.1016/S0017-9310(98)00098-2.

[73] M. J. Yu, S.W. Baek, J. H. Park, Int. J. Heat Mass Tran., 2000, 43, 1699-1713, doi: 10.1016/S0017-9310(99)00265-3.

[74] C. Yin, L. C. R. Johansen, L. Rosendahl, S. K. Kær, Energ. Fuel., 2010, 24, 6275-6282, doi: 10.1021/ef101211p.

[75] L. J. Dorigon, G. Duciak, R. Brittes, F. Cassol, M. Galarça, F.H.R. França, Int. J. Heat Mass Tran., 2013, 64, 863-873, doi: 10.1016/j.ijheatmasstransfer.2013.05.010.

[76] M. K. Denison, B.W. Webb, Int.. J. Heat Tran., 1993, 115, 1004-1012, doi: 10.1115/1.2911354.

[77] M. K. Denison, B.W. Webb, Int. J. Heat Mass Tran., 1995, 38, 1813-1821, doi: 10.1016/0017-9310(94)00297-9.

[78] M. K. Denison, B. W. Webb, J. Heat Tran., 1995, 117, 359365, doi: 10.1115/1.2822530.

[79] V. P. Solovjov, D. Lemonnier, B.W. Webb, J. Heat Tran., 2010, 133, 821-830, doi: 10.1115/IHTC14-22299.

[80] V. P. Solovjov, D. Lemonnier, B.W. Webb, J. Quant. Spectrosc. Ra., 2011, 112, 1205-1212, doi: 10.1016/j.jqsrt.2010.08.009.

[81] J.T. Pearson, B.W. Webb, V.P. Solovjov, J. Ma, J. Quant. Spectrosc. Ra., 2013, 128, 10-17, doi: 10.1016/j.jqsrt.2012.07.016.

[82] J. T. Pearson, B. W. Webb, V. P. Solovjov, J. Ma, J. Quant. Spectrosc. Ra., 2014, 138, 82-96, doi: 10.1016/j.jqsrt.2014.01.019.

[83] V. P. Solovjov, F. Andre, D. Lemonnier, B. W. Webb, J. Phys. Conf. Ser., 2016, 676, 012022, doi: 10.1088/17426596/676/1/012022.

[84] V. P. Solovjov, F. Andre, D. Lemonnier, B.W. Webb, J. Quant. Spectrosc. Ra., 2018, 206, 198-212, doi: 10.1016/j.jqsrt.2017.11.012.

[85] V. P. Solovjov, B.W. Webb, F. Andre, J. Quant. Spectrosc. Ra., 2018, 214, 120-132, doi: 10.1016/j.jqsrt.2018.04.026.

[86] M. F. Modest, J. Heat Tran., 2013, 135, 061801, doi: $10.1115 / 1.4023596$.

[87] M.F. Modest, R.J. Riazzi, J. Quant. Spectrosc. Ra., 2005, 90, 169-189, doi: 10.1016/j.jqsrt.2004.03.007. 
[88] C. Wang, W. Ge, M.F. Modest, B. He, J. Quant Spectrosc. $R a$., 2016, 168, 46-56, doi: 10.1016/j.jqsrt.2015.08.017.

[89] C. Wang, M. F. Modest, B. He, J. Quant. Spectrosc. Ra., 2016, 176, 129-136, doi: 10.1016/j.jqsrt.2016.02.007.

[90] C. Wang, M.F. Modest, B. He, Int. J. Therm. Sci., 2016, 108, 100-107, doi:10.1016/j.ijthermalsci.2016.05.005.

[91] C. Wang, B. He, M.F. Modest, T. Ren, J. Quant. Spectrosc. Ra., 2018, 219, 108-116, doi: _10.1016/j.jqsrt.2018.04.002.

[92] S. Zheng, R. Sui, Y. Yang, Y. Sun, H. Zhou, Q. Lu, Int. Commun. Heat Mass Tran., 2020, 114, 104566, doi: 10.1016/j.icheatmasstransfer.2020.104566.

[93] H. Chu, M. Gu, H. Zhou, F. Liu, Front. Energy, 2014, 8, 4148, doi: 10.1007/S11708-013-0292-4.

[94] H. Chu, F. Liu, H. Zhou, Int. J. Therm. Sci., 2012, 59, doi: 10.1016/j.ijthermalsci.2012.04.003.

[95] F. Liu, J. Heat Trans-T. ASME, 1999, 121, 200-203, doi: 10.1115/1.2825944.

[96] F. Liu, G. J. Smallwood, O. L. Gülder, Int. J. Heat Mass Tran., 2000, 43, 3119-3135, doi: 10.1016/S0017-9310(99)003439.

[97] F. Liu, G. J. Smallwood, Ö. L. Gülder, J. Quant. Spectrosc. $R a ., 2001,68$, 401-417, doi: 10.1016/S0022-4073(00)00033-9.

[98] L. Yan, G. Yue, B. He, Appl. Therm. Eng., 2016, 94, 209-220, doi: 10.1016/j.applthermaleng.2015.09.109.

[99] H.C. Hottel, E.S. Cohen, AICHE J., 1958, 4, 3-14, doi: 10.1002/AIC.690040103.

[100] J. T. Farmer, J. R. Howell, J. Thermophys. Heat Tran., 1994, 8, 133-139, doi: $10.2514 / 3.511$.

[101] J. R. Howell, Advan. Heat Tran., 1969, 5, 1-54, doi: 10.1016/S0065-2717(08)70128-X.

[102] J.R. Howell, M. Perlmutter, J. Heat Trans-T. ASME, 1964, 86, 116-122, doi: 10.1115/1.3687044.

[103] F. C. Lockwood, N. G. Shah, A new radiation solution method for incorporation in general combustion prediction procedures, in, 1981, pp. 1405-1414.

[104] M. P. Mengüç, R. Viskanta, J. Quant. Spectrosc. Ra., 1985, 111, 533-549, doi: 10.1016/J.JQSRT.2010.01.025.

[105] M. F. Modest, J. Heat Trans-T. ASME, 1990, 112, 819-821, doi: $10.1115 / 1.2910468$.

[106] J.C. Chai, H.S. Lee, S.V. Patankar, J.Appl. Anim. Res., 1993, 24, 373-389, doi: 10.1080/10407799308955899.

[107] P.J. Coelho, J. Quant. Spectrosc. Ra., 2002, 73, 231-238, doi: 10.1016/S0022-4073(01)00202-3.

[108] P. J. Coelho, Comput. Mech., 2004, 33, 375-388, doi: 10.1007/S00466-003-0539-8.

[109] W.A. Fiveland, J. Thermophys. Heat Tr., 1988, 2, 309-316, doi: $10.2514 / 3.105$.

[110] Z. Guo, S. Kumar, J. Thermophys. Heat Tr., 2002, 16, $289-$ 296, doi: 10.2514/2.6689.

[111] K. H. Lee, R. Viskanta, Numer. Heat Tr. A-Appl., 2001, 39, 205-225, doi: 10.1080/104077801300006553.

[112] H.-S. Li, G. Flamant, J.-D. Lu, Mitigation of ray effects in the discrete ordinates method, Numer. Heat Tr. B-Fund., 2003, 43, 445-466, doi: 10.1080/713836241.
[113] J. C. Chai, H. S. Lee, S.V. Patankar, J. Thermophys. Heat Tr., 1994, 8, 419-425, doi: 10.2514/3.559.

[114] J.C. Chai, H.S. Lee, S.V. Patankar, Numer. Heat Tr. B-Fund., 1994, 26, 225-235, doi: 10.1080/10407799408914927.

[115] J.C. Chai, G. Parthasarathy, H.S. Lee, S.V. Patankar, J. Thermophys. Heat Tr., 1994, 9, 410-415, doi: 10.2514/3.682.

[116] G. D. Raithby, E. H. Chui, J. Heat Trans-T. ASME, 1990, 112, 415-423, doi: 10.1115/1.2910394.

[117] S. Chandrasekhar, Radiative transfer, Courier Corporation, 2013.

[118] W.A. Fiveland, J. Heat Trans-Tasme., 1984, 106, 699-706, doi: $10.1115 / 1.3246741$.

[119] J. Truelove, J. Quant. Spectrosc. Ra., 1988, 39, 27-31, doi: 10.1016/0022-4073(88)90016-7.

[120] P. Asinari, S.C. Mishra, R. Borchiellini, Numer. Heat Tr. BFund., 2010, 57, 126-146, doi: 10.1080/10407791003613769.

[121] S.C. Mishra, H. Poonia, R.R. Vernekar, A.K. Das, Heat Transfer. Eng., 2014, 35, 1267-1278, doi: 10.1080/01457632.2013.876806.

[122] Y. Zhang, H. Yi, H. Tan, Opt. Express, 2013, 21, 2453224549, doi: 10.1364/OE.21.024532.

[123] Y. Zhang, Y. Ma, H.-L. Yi, H.-P. Tan, J. Quant. Spectrosc. Ra., 2013, 129, 118-130, doi: 10.1016/J.JQSRT.2013.06.002.

[124] Y. Zhang, H.-L. Yi, H.-P. Tan, Int. J. Heat Mass Tran., 2013, 56 , 411-423,

doi:

10.1016/J.IJHEATMASSTRANSFER.2012.09.051.

[125] Y. Zhang, H.-L. Yi, H.-P. Tan, J. Comput. phys., 2013, 241, 18-34, doi: 10.1016/J.JCP.2013.01.044.

[126] C. A. Wang, H. Sadat, V. Ledez, D. Lemonnier, Int. J. Therm. Sci., 2010, 49, 2282-2288, doi: 10.1016/j.ijthermalsci.2010.06.024.

[127] C.A. Wang, H. Sadat, J.Y. Tan, Numer. Heat Tr. B-Fund., 2014, 65, 518-536, doi: 10.1080/10407790.2013.873305.

[128] H.-S. Li, G. Flamant, J.-D. Lu, Int. Commun. Heat Mass , 2003, 30, 61-70, doi: 10.1016/S0735-1933(03)00008-3.

[129] H.-S. Li, H.C.H.-C. Zhou, J.D.J.-D. Lu, C.G.C.-G. Zheng, J. Quant. Spectrosc. Ra., 2003, 78, 437-453, doi: 10.1016/S00224073(02)00279-0.

[130] C.T. Salinas, Int. J. Therm. Sci., 2010, 49, 302-310, doi: 10.1016/j.ijthermalsci.2009.08.003.

[131] H. Zhou, Q. Cheng, The DRESOR method for the solution of the radiative transfer equation in gray plane-parallel media, in: ICHMT DIGITAL LIBRARY ONLINE, Begel House Inc., 2004. [132] H.-C. Zhou, D.-L. Chen, Q. Cheng, J. Quant. Spectrosc. Ra., 2004, 83, 459-481, doi: 10.1016/S0022-4073(03)00031-1.

[133] J. Yang, H, Wang, A. Liu, Heat Transf. Res., 2020, 7, 623639.

[134] Z.F. Huang, Q. Cheng, C. He, H.C. Zhou, Heat Transf. Res., 2008, 37, 138-152, doi: 10.1002/htj.20198.

[135] H.-C. Zhou, Q. Cheng, Z.-F. Huang, C. He, J. Quant. Spectrosc. $\quad R a$., 2007, 104, 99-115, doi: 10.1016/j.jqsrt.2006.08.015.

[136] Q. Cheng, H.-C. Zhou, Y.-1. Yu, D.-x. Huang, Numer. Heat Tr. B-Fund., 2008, 54, 354-367, doi: $10.1080 / 10407790802358824$. 
[137] Z. Huang, H. Zhou, Q. Cheng, P.-f. Hsu, Int. J. Heat Mass Tran., 2013, 60, 81-87, doi: 10.1016/j.ijheatmasstransfer.2012.12.056.

[138] S. Zheng, C. Qi, Z. Huang, H. Zhou, J. Quant. Spectrosc. Ra., 2018, 217, 425-431, doi: 10.1016/j.jqsrt.2018.06.020.

[139] R. M. Goody, Y. L. Yung, Atmospheric radiation: theoretical basis, Oxford university press, 1995.

[140] S.S. Penner, Quantitative molecular spectroscopy and gas emissivities, Addison-Wesley Publishing Company, 1959.

[141] S.J. Young, J. Quant. Spectrosc. Ra., 1977, 18, 1-28, doi: 10.1016/0022-4073(77)90125-X.

[142] W. Godson, Q. J. R. Meteorol. Soc., 1953, 79, 367-379, doi: 10.1002/qj.49707934104.

[143] T. K. Kim, J. A. Menart, H. S. Lee, J. Heat Trans-Tasme., 1991, 113, 946-952, doi: 10.1115/1.2911226.

[144] F. Liu, Ö.L. Gülder, G. J. Smallwood, Y. Ju, Int. J. Heat Mass Tran., 1998, 41, 2227-2236, doi: 10.1016/S00179310(97)00267-6.

[145] V. Goutiere, F. Liu, A. Charette, J. Quant. Spectrosc. Ra., 2000, 64, 299-326, doi: 10.1016/S0022-4073(99)00102-8.

[146] J. Taine, A. Soufiani, Adv. Heat Transf., 1999, 33, 44-51, doi: 10.1016/S0065-2717(08)70306-X.

[147] C. Hua Qiang, F. Yan, C. WenJian, R. Fei, G. MingYan, Acta Phys. Sin-ch. Ed., 2017, 66, 094207, doi: 10.7498/aps.66.094207.

[148] M.H. Bordbar, G. Węcel, T. Hyppänen, Combust. Flame, 2014, 161, 2435-2445, doi: 10.1016/j.combustflame.2014.03.013. [149] R. Johansson, B. Leckner, K. Andersson, F. Johnsson, , Combust. Flame, 2011, 158, 893-901, doi: 10.1016/j.combustflame.2011.02.001.

[150] L.R. Zhou, Gui Rong Yan, Jin Ping Ou, Comput-Aided Civ. Inf., 2013, 28, 210-226, doi: 10.1111/j.1467-8667.2012.00803.x

[151] E.R. Van Dam, B. Husslage, D. Den Hertog, H. Melissen, Oper. Res., 2007, 55, 158-169, doi: 10.2307/25147062.

[152] E. Mancaruso, M. Lavorgna, B.M. Vaglieco, F. Taglialatelascafati, Nonlinear Systems and Circuits in Internal Combustion Engines, Springerbriefs in Applied Sciences \& Technology, (2018).

[153] V. P. Solovjov, B.W. Webb, J. Heat Trans-Tasme., 2011, 133, doi: 10.1115/1.4002775.

[154] R. J. Howell, J. Heat Trans-Tasme, 1998, 120, 547, doi: 10.1115/1.2824310.

[155] Q. Cheng, H.-C. Zhou, J. Heat Trans-Tasme., 2006, 129, 634-645, doi: 10.1115/1.2712477.

[156] H.-C. Zhou, F. Sheng, S.-D. Han, C.-G. Zheng, Numer. Heat Tr. A: Appl., 2000, 38, 757-773, doi: 10.1080/10407780050195689.

[157] P. Rivière, S. Langlois, A. Soufiani, J. Taine, J. Quant. Spectrosc. Ra., 1995, 53, 221-234, doi: 10.1016/00224073(95)90009-8.

[158] D. Scutaru, L. Rosenmann, J. Taine, J. Quant. Spectrosc. Ra., 1994, 52, 765-781, doi: 10.1016/0022-4073(94)90042-6.

[159] J.-M. Flaud, C. Camy-Peyret, R.A. Toth, Paramètres des raies de la vapeur d'eau des micro-ondes à l'infrarouge moyen, Pergamon Press, Oxford, (1981)
[160] C. Chackerian, R.H. Tipping, J. Mol. Spectrosc., 1983, 99, 431-449, doi: 10.1016/0022-2852(83)90324-7.

[161] G. Guelachvili, D. de Villeneuve, R. Farrenq, W. Urban, J. Verges, J. Mol. Spectrosc., 1983, 98, 64-79, doi: 10.1016/00222852(83)90203-5.

[162] M.-Y. Perrin, A. Soufiani, J. Quant. Spectrosc. Ra., 2007, 103, 3-13, doi: 10.1016/j.jqsrt.2006.07.018.

[163] K. Wakatsuki, High Temperature Radiation Absorption of Fuel Molecules And An Evaluation of Its Influence on Pool Fire Modeling, Ph.D. thesis, University of Maryland, USA, (2005).

[164] K. Wakatsuki, S.P. Fuss, A. Hamins, M.R. Nyden, P. Combust. Inst., 2005, 30, 1565-1573, doi: 10.1016/j.proci.2004.08.078.

[165] K. Wakatsuki, G. S. Jackson, A. Hamins, M.R. Nyden, $P$. Combust. Inst., 2007, 31, 2573-2580, doi: 10.1016/j.proci.2006.08.049.

[166] M.A. Brosmer, C.L. Tien, J. Quant. Spectrosc. Ra., 1985, 33, 521-532, doi: 10.1016/0022-4073(85)90137-2.

[167] J.L. Consalvi, R. Demarco, A. Fuentes, Combust. Theor. Model., 2012, 16, 817-841, doi: 10.1080/13647830.2012.662283. [168] J.L. Consalvi, F. Liu, Combust. Sci. Technol., 2014, 186, 475-489, doi: 10.1080/00102202.2014.883221.

[169] H. Guo, F. Liu, G.J. Smallwood, O.L. Gulder, Combust. Theor. Model., 2002, 6, 173-187, doi: 10.1088/1364$7830 / 6 / 2 / 301$

[170] F. Liu, G. J. Smallwood, W. Kong, J. Quant. Spectrosc. Ra., 2011, 112, 1241-1249, doi: 10.1016/j.jqsrt.2010.08.021.

[171] B. Ribi, M.P. Meyer, Influence of Gap Between Casing and Variable Stator Blade on Axial Compressor Performance, in, ASME Turbo Expo 2008: Power for Land, Sea, and Air, 2008, pp. 203-217.

[172] C. Yin, L.C.R. Johansen, L.A. Rosendahl, S.K. Kær, Energ. Fuel., 201, 24, 6275-6282, doi: 10.1021/ef101211p.

[173] T. Kangwanpongpan, F.H.R. França, R. Corrêa da Silva, P.S Schneider, H.J. Krautz, Int. J. Heat Mass Tran., 2012, 55, 74197433, doi: 10.1016/j.ijheatmasstransfer.2012.07.032.

[174] S. Zheng, Y. Yang, H. Zhou, Int. J. Heat Mass Tran., 2019, 129, 1232-1241, doi: 10.1016/j.ijheatmasstransfer.2018.10.067.

[175] J. Ma, B.-W. Li, J.R. Howell, Int. J. Heat Mass Tran., 2014, 71, 35-43, doi: 10.1016/j.ijheatmasstransfer.2013.12.009.

[176] N.W. Bressloff, Int. J. Heat Mass Tran., 1999, 42, 34693480, doi: 10.1016/S0017-9310(99)00031-9.

[177] J. S. Truelove, Zone method for radiative heat transfer calculations, HTFS DR33, AERE, Harwell, Oxon, UK, (1975).

[178] R. R. Burrell, J. L. Pagliaro, G.T. Linteris, P. Combust. Inst., 2019, 37, 4231-4238, doi: 10.1016/j.proci.2018.06.018.

[179] E.C. Okafor, Y. Naito, S. Colson, A. Ichikawa, T. Kudo, A. Hayakawa, H. Kobayashi, Combust. Flame, 2019, 204, 162-175, doi: 10.1016/j.combustflame.2019.03.008.

[180] W. Han, Z. Chen, Combust. Flame, 2015, 162, 2128-2139, doi: 10.1016/j.combustflame.2015.01.011.

[181] W. Han, Z. Chen, Int. J. Heat Mass Tran., 2106, 99, 691701, doi:10.1016/j.ijheatmasstransfer.2016.04.003.

[182] R. K. Mehra, H. Duan, S. Luo, F. Ma, Fuel, 2019, 246, 476490, doi: 10.1016/j.fuel.2019.03.003. 
[183] Y. Sun, S. Zheng, B. Jiang, J. Tang, F. Liu, Int. J. Heat Mass Tran., 2019, 145, 118777, doi: 10.1016/j.ijheatmasstransfer.2019.118777.

[184] A. Soufiani, E. Djavdan, Combust. Flame, 1994, 97, 240250, doi: 10.1016/0010-2180(94)90007-8.

[185] H. Amiri, K. Lari, P.J. Coelho, Int. J. Therm. Sci., 2017, 118, 448-460, doi: 10.1016/j.ijthermalsci.2017.05.014.

[186] F. Liu, J. Heat Tran-Tasme.,1999, 121, 200-203, doi: 10.1115/1.2825944.

[187] Y. Yang, S. Zheng, Q. Lu, Int. J. Therm. Sci., 2021, 161, 06783, doi: 10.1016/j.ijthermalsci.2020.106783.

[188] G. C. Fraga, H. Bordbar, S. Hostikka, F.H.R. França, J. Heat Tran-Tasme., 2020, 142, doi: 10.1115/1.4045666.

[189] C.P. Thurgood, A. Pollard, H.A. Becker, J. Heat TranTasme., 1995, 117, 1068-1070, doi: 10.1115/1.2836285.

[190] Y. Sun, J. Ma, Y. Yu, B. Ye, C. Gao, J. Quant. Spectrosc. Ra., 2019, 236, 106600, doi: 10.1016/j.jqsrt.2019.106600.

[191] Y. Zhou, C. Wang, T. Ren, J. Quant. Spectrosc. Ra., 2020, 254, 107199, doi: 10.1016/j.jqsrt.2020.107199.

\section{Author information}

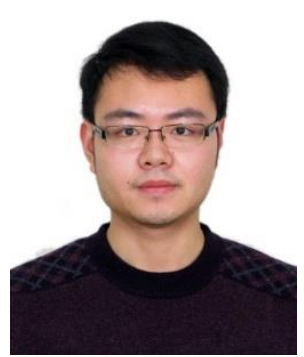

Shu Zheng received the bachelor's and Ph.D. degrees from the Huazhong University of Science and Technology, Wuhan, China, in 2009 and 2013, respectively. From 2013 to 2016, he was a Post-Doctoral Researcher in Department of Thermal Engineering, Tsinghua University. He is currently an Associate Professor with the National Engineering Laboratory for Biomass Power Generation Equipment, North China Electric Power University, Beijing, China. His current research interests include radiation heat transfer analysis and combustion diagnostics.

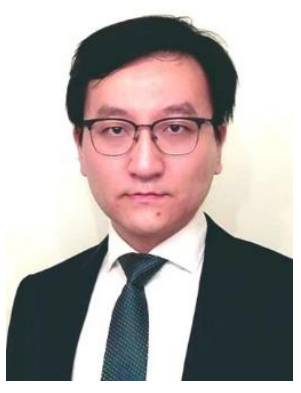

Ran Sui is an Assistant Professor of Mechanical and Aerospace Engineering at Missouri University of Science and Technology. Prior to Missouri S\&T, he was an Associate Research Scholar (2020) and a Postdoctoral Fellow (2018) at Princeton University. He received his Ph.D. degree in Mechanical Engineering from ETH Zurich in 2017. His main research interests include catalytic combustion, coupling of hetero-/homogeneous thermochemical processes, and the related computational methods and experimental diagnostics.

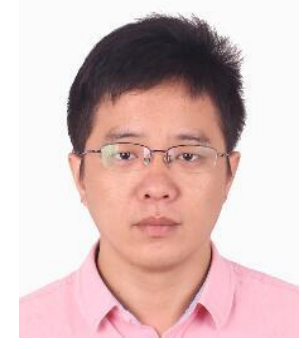

Yujia Sun received the bachelor's and Ph.D. degrees from Nanjing University of Science and Technology, Nanjing, China, in 2012 and 2018, respectively. He is currently an Associate Professor with School of Atmospheric Physics, Nanjing University of Information Science and Technology, Nanjing, China His current research interests include radiation heat transfer and combustion modeling.

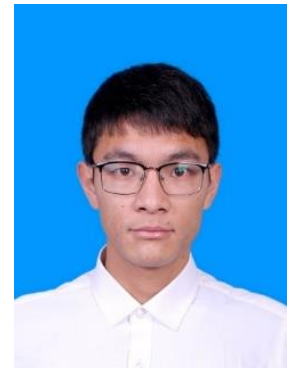

Yu Yang received the bachelor's and master's degrees from the North China Electric Power University, Beijing, China, in 2017 and 2020, respectively. He is currently a doctoral student at North China Electric Power University, Beijing, China. His current research interests include radiation heat transfer and combustion modeling.

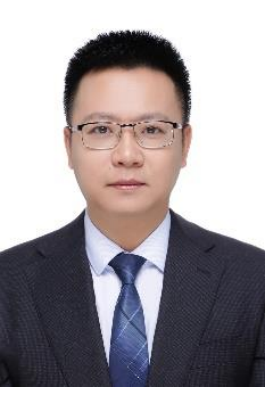

Qiang $\mathbf{L u}$ received the bachelor's and Ph.D. degrees from the University of Science and Technology of China, Hefei, China, in 2005 and 2010, respectively. He is currently a Professor with the National Engineering Laboratory for Biomass Power Generation Equipment, North China Electric Power University, Beijing, China. He was awarded the second prize of National Science and Technology Progress Award in 2019, the first prize of Science and Technology Progress Award by the Ministry of Education in 2016 and 2018, the National Science Fund for Excellent Young Scholars in 2019, and honored as a national "Ten Thousand Plan" Young Talent in 2019. His current research interests include radiation heat transfer and thermochemical conversion of biomass to produce fuels and chemicals.

Publisher's Note: Engineered Science Publisher remains neutral with regard to jurisdictional claims in published maps and institutional affiliations. 